\title{
Well-posedness analysis of multicomponent incompressible flow models
}

\author{
Dieter Bothe(D) AND Pierre- Etienne Druetid
}

\begin{abstract}
In this paper, we extend our study of mass transport in multicomponent isothermal fluids to the incompressible case. For a mixture, incompressibility is defined as the independence of average volume on pressure, and a weighted sum of the partial mass densities stays constant. In this type of models, the velocity field in the Navier-Stokes equations is not solenoidal and, due to different specific volumes of the species, the pressure remains connected to the densities by algebraic formula. By means of a change of variables in the transport problem, we equivalently reformulate the PDE system as to eliminate positivity and incompressibility constraints affecting the density, and prove two type of results: the local-in-time well-posedness in classes of strong solutions, and the global-in-time existence of solutions for initial data sufficiently close to a smooth equilibrium solution.
\end{abstract}

\section{Multicomponent diffusion in an incompressible fluid}

In this paper, we study the well-posedness analysis in classes of strong solutions of class-one models ${ }^{1}$ of mass transport in isothermal, incompressible multicomponent fluids. This investigation is a direct continuation of results obtained recently concerning the compressible case in [5], and the weak solvability of the incompressible model in [14]. Performing the incompressible limit (the low-Mach number limit) in models for fluid mixtures and for multicomponent fluids is desirable both from the practical and the theoretical viewpoint. On the one hand, fluid mixtures occurring in applications are often incompressible, and the limit passage reduces the stiffness of the models by eliminating the parameter which is practically infinite. On the other hand, the lowMach number limit leads to a type of incompressibility condition which has not yet been studied in the context of mathematical analysis for fluid dynamical equations.

We are interested in the second type of issue, that is, the theoretical issues of unique solvability and continuous dependence in classes of strong solutions for the underlying PDEs. The model class for multicomponent transport in fluids here under study is

Mathematics Subject Classification: 35M33, 35Q30, 76N10, 35D35, 35B65, 35B35, 35K57, 35Q35, 35Q79, 76R50, 80A17, 80A32, 92E20

Keywords: Multicomponent flow, Complex fluid, Fluid mixture, Incompressible fluid, Low Machnumber, Strong solutions.

The second author gratefully acknowledges the Grant DR1117/1-1 of the German Science Foundation.

${ }^{1}$ Class-one is a terminology that we adopt from the paper [2] to describe the class of multicomponent flow models with single common velocity and temperature. The concept goes back to the work of C. Hutter. 
the one proposed in [2], also applied to mixtures with charged constituents in [12, 13]. Concerning the fundamentals of thermodynamics for fluid mixtures, the reader is referred to these papers, or to the book [16]. The model for Mach-number zero (incompressibility constraint) is based on I. Müller's definition of incompressibility as invariance of the volume under pressure variations [17,25]. More directly, we follow the recent example of [12] (formal limit), and the more general road map proposed in the Section 16 of [2]. In [3] we propose a derivation of the incompressible limit starting from a few postulates of mathematical nature about the structure of the Helmholtz free energy. Similar concepts have been exposed and discussed in a few research papers like [10,19,24]. Incompressible mixtures are also conceptualised in the book [27]. The corner stone of these works is that incompressibility for a multicomponent system means the invariance of average volume under pressure variations. For a fluid mixture of $N \geq 2$ chemical species $\mathrm{A}_{1}, \ldots, \mathrm{A}_{N}$, it assumes the form of a volume constraint

$$
\sum_{i=1}^{N} \rho_{i} \bar{V}_{i}=1
$$

where $\bar{V}_{1}, \ldots, \bar{V}_{N}>0$ are partial specific volumes of the molecules at reference temperature and pressure. The relation generalises the assumption of a constant mass density considered in other analytical investigations, a. o. $[6,8,18,22]$. In the present paper we are interested only in the general case that at least two indices exist such that $\bar{V}_{i_{1}} \neq \bar{V}_{i_{2}}$ or, in vectorial notation, that $\bar{V} \neq \lambda 1^{N}$ for all $\lambda \in \mathbb{R}$, where $\bar{V}=$ $\left(\bar{V}_{1}, \bar{V}_{2}, \ldots, \bar{V}_{N}\right)$ and $1^{N}=(1,1, \ldots, 1) \in \mathbb{R}^{N}$.

Bulk. The convective and diffusive mass transport of these species and the momentum balance are described by the partial differential equations

$$
\begin{aligned}
& \partial_{t} \rho_{i}+\operatorname{div}\left(\rho_{i} v+J^{i}\right)=r_{i} \\
& \text { for } i=1, \ldots, N \text {, } \\
& \partial_{t}(\varrho v)+\operatorname{div}(\varrho v \otimes v-\mathbb{S}(\nabla v))+\nabla p=\sum_{i=1}^{N} \rho_{i} b^{i}(x, t) .
\end{aligned}
$$

The physical system is assumed isothermal with absolute temperature $\theta>0$. The partial mass densities of the species are denoted $\rho_{1}, \ldots, \rho_{N}$. Throughout the paper we shall use the abbreviation $\varrho:=\sum_{i=1}^{N} \rho_{i}$ for the total mass density. The barycentric velocity of the fluid is called $v$ and the thermodynamic pressure $p$. In the Navier-Stokes equations, $\mathbb{S}(\nabla v)$ denotes the viscous stress tensor, which we assume for simplicity of Newtonian form. The vector fields $b^{1}, \ldots, b^{N}$ are the external body forces. The diffusions fluxes $J^{1}, \ldots, J^{N}$, that are defined to be the non-convective part of the mass fluxes, must satisfy by definition the necessary side-condition $\sum_{i=1}^{N} J^{i}=0$. A thermodynamic consistent Fick-Onsager closure respecting this constraint is assumed. This approach is described in great generality among others by [2,13] following older 
ideas by $[9,23]$. The diffusions fluxes $J^{1}, \ldots, J^{N}$ obey

$$
J^{i}=-\sum_{j=1}^{N} M_{i, j}\left(\rho_{1}, \ldots, \rho_{N}\right)\left(\nabla \mu_{j}-b^{j}\right) \text { for } i=1, \ldots, N .
$$

The Onsager matrix $M\left(\rho_{1}, \ldots, \rho_{N}\right)$ is a symmetric, positive semi-definite $N \times N$ matrix for every $\left(\rho_{1}, \ldots, \rho_{N}\right) \in \mathbb{R}_{+}^{N}$. In all known linear closure approaches, this matrix satisfies

$$
\sum_{i=1}^{N} M_{i, j}\left(\rho_{1}, \ldots, \rho_{N}\right)=0 \text { for all }\left(\rho_{1}, \ldots, \rho_{N}\right) \in \mathbb{R}_{+}^{N} .
$$

One possibility to compute the special form of $M$ is for instance to invert the MaxwellStefan balance equations. For the mathematical treatment of this algebraic system, the reader can consult $[1,16,18,20,22]$. Or $M$ is constructed directly in the form $P^{\top} M_{0} P$, where $M_{0}$ is a given matrix of full rank, and $P$ is a projector guaranteeing that (5) is valid. The paper [4] establishes equivalence relations between the Fick-Onsager and the Maxwell-Stefan constitutive approaches, proposing moreover a novel unifying approach to close the diffusion model.

The quantities $\mu_{1}, \ldots, \mu_{N}$ are the chemical potentials from which the thermodynamic driving forces for the diffusion phenomena are inferred. For an incompressible system, they are related to the mass densities $\rho_{1}, \ldots, \rho_{N}$ and to the pressure via

$$
\mu_{i}=\bar{V}_{i} p+\partial_{\rho_{i}} k\left(\theta, \rho_{1}, \ldots, \rho_{N}\right) .
$$

Here the function $k$ denotes the positively homogeneous part of the free energy. It can be characterised as $k=\varrho \psi^{0}$, where $\psi^{0}$ is the specific free energy at reference pressure $p_{0}$. We refer to the second section of the paper [3] for general representation results for the free energy function from available data. A particular, but typical choice discussed among others in [2] is

$$
k(\theta, \rho)=\sum_{i=1}^{N} \mu_{i}^{\mathrm{ref}} \rho_{i}+k_{B} \theta \sum_{i=1}^{N} n_{i} \ln y_{i}
$$

where $n_{i}:=\rho_{i} / m_{i}$ are the number densities with the molecular masses $m_{1}, \ldots, m_{N}>$ $0, y_{i}=n_{i} / \sum_{j=1}^{N} n_{j}$ are the number fractions, and $\mu_{i}^{\text {ref }}$ are reference values of the chemical potentials. For the mathematical theory in this paper, more general structures in (7) will however be admitted. The isothermal Gibbs-Duhem equation: $d p=\sum_{i=1}^{N} \rho_{i} d \mu_{i}$ defines the intrinsic relationship between (1), (6), and the pressure field. The paper [3] shows that the relation (6) indeed occurs in the limit case when the bulk free energy density of the system adopts the singular form

$$
\varrho \psi=h^{\infty}(\theta, \rho):= \begin{cases}k(\theta, \rho) & \text { if } \sum_{i=1}^{N} \rho_{i} \bar{V}_{i}=1, \\ +\infty & \text { otherwise }\end{cases}
$$


The relation (6) is an equivalent expression of $\mu \in \partial h^{\infty}(\theta, \rho)$, where $\partial$ denote the subdifferential of the convex function $h^{\infty}(\theta, \cdot)$, and the function $p=-h^{\infty}(\theta, \rho)+$ $\sum_{i=1}^{N} \rho_{i} \mu_{i}$ can be understood as a 'Lagrange multiplier' associated with the constraint (1).

We notice that, multiplying the equations (2) with the constants $\bar{V}_{i}$ and summing up, the local change of volume is described by the equation

$$
\operatorname{div} v=-\operatorname{div}\left(\sum_{i=1}^{N} \bar{V}_{i} J^{i}\right)+\sum_{i=1}^{N} \bar{V}_{i} r_{i} .
$$

Effects like diffusion and chemical reactions will induce a local change in the molecular composition, implying a net local change of the volume, independent of a mechanical compression or expansion.

Concerning the presence of reaction terms in (2), we have to mention in respect with the compressible systems considered in [5] a subtle difference of the incompressible models. For the compressible case, the reactions densities $r_{i}$ in (2) are allowed to be general functions $r_{i}=r_{i}\left(\rho_{1}, \ldots, \rho_{N}\right)$, without influencing qualitatively the wellposedness results or the mathematical methods. This is different in the incompressible case. At first, the restriction (1) implies that $\mu$ does not depend on $\rho$ only, so that the structure $r=r(\rho)$ does not comply with standard thermodynamically consistent reaction terms. At second, the 'elliptic equation' (9) defines a differential operator acting on a certain relative chemical potential (variable $\zeta$, details below). This elliptic operator is linear for the pure diffusion case, but turns to nonlinear in the presence of reactions of the general form $r=r(\mu)$. In this paper, we treat incompressible multicomponent diffusion in itself. We shall address the specific problems raised by chemical reactions in further research. Thus, allowing - as we shall do-for certain source terms $r=r(\rho)$ in (2) means a bit more mathematical generality, but it remains clear that realistic models of chemical reactions require nontrivial modifications of the methods used here.

As to the stress tensor $\mathbb{S}$ we shall restrict for simplicity to the standard Newtonian form with constant coefficients. However, we present methods which are sufficient to extend the results to the case of density and composition dependent viscosity coefficients. Pressure dependence of the coefficients is not really meaningful at zero compressibility, since the partial mass densities turn independent on pressure (cf. the incompressible asymptotics in [3]).

Boundary and initial conditions. We investigate the problem (2), (3) in a cylindrical domain $\left.Q_{T}:=\Omega \times\right] 0, T\left[\right.$ where $T$ is a finite time and $\Omega \subset \mathbb{R}^{3}$ a bounded domain. It is possible to treat the case $\Omega \subset \mathbb{R}^{d}$ for general $d \geq 2$ with similar methods. We consider initial conditions

$$
\begin{array}{ll}
\rho_{i}(x, 0)=\rho_{i}^{0}(x) & \text { for } x \in \Omega, i=1, \ldots, N, \\
v_{j}(x, 0)=v_{j}^{0}(x) & \text { for } x \in \Omega, j=1,2,3 .
\end{array}
$$


For simplicity, we consider the linear homogeneous boundary conditions

$$
\begin{aligned}
& \left.v=0 \quad \text { on } S_{T}:=\partial \Omega \times\right] 0, T[, \\
& v \cdot J^{i}=0 \quad \text { on } S_{T} \text { for } i=1, \ldots, N .
\end{aligned}
$$

In addition to the no-slip boundary condition (12), it would be interesting to consider also other relevant choices, like partial split, inflow or pressure boundary conditions. However, our main purpose in this article is to obtain a first result for incompressible flow problems in the multicomponent case. To this aim the condition (12), which is well-known among mathematicians, provides a good starting example. Other boundary conditions would moreover oblige us to introduce more functional-space framework to treat the traces. This will have to be the interesting subject of future work.

As a matter of fact, these simplifying choices oblige us to make a further restriction. To see this, we recall the relation (9), that we integrate over $\Omega$. If there is no mass flux through the boundary, we see that $\int_{\Omega} \sum_{i=1}^{N} \bar{V}_{i} r_{i}(x, t) d x=0$. This condition cannot be enforced for a general $r=r(\rho)$, unless we assume that $r$ takes values in $\{\bar{V}\}^{\perp}$. Recalling that realistic models for chemical reactions are to be treated in an upcoming paper, we here restrict to the case that $r(\rho) \cdot \bar{V}=0$ for all $\rho$.

\section{State of the art and our main result}

\subsection{A review of prior investigations and our method}

Up to few exceptions, models for incompressible multicomponent fluids have not been investigated in mathematical analysis. For a mathematical treatment in the case of the constraint $\varrho=$ const, which corresponds to choosing $\bar{V}_{1}=\cdots=\bar{V}_{N}$ in (1), the reader might consult the papers [8] and [22] (global weak solution analysis) and [18], and [6] (local-in-time well-posedness). ${ }^{2}$ From the viewpoint of the mathematical structure, the case $\varrho=$ const exhibits profoundly different features than the general relation (1). The principal difference is that (4), (5) and (6) imply the decoupling of the pressure and of the diffusion fluxes. The Navier-Stokes equations reduce to their single component solenoidal variant and can be solved independently. Of course, this does not mean that $\varrho=$ const cannot be a good approximation under special circumstances. In [7] for instance, a class of multicomponent mixtures has been introduced for which the use of the incompressible Navier-Stokes equation is realistic: Incompressibility is assumed for the solvent only, and diffusion is considered against the solvent velocity. See also the discussion in the paragraph 4.8 of [27] on incompressible mixtures.

In the case that $\bar{V}$ is not parallel to $1^{N}$, (4) implies that the pressure affects the diffusion fluxes via the chemical potentials. A corollary of this fact is that if we multiply the equations (2) with the constants $\bar{V}_{i}$ and sum up, we obtain (9) for the local change of volume. Moreover,

\footnotetext{
${ }^{2}$ In the latter paper the phase change liquid/gas is actually in the focus. All references are based on the equivalent Maxwell-Stefan structure for the diffusion fluxes, rather than the Fick-Onsager one.
} 
(a) the viscous stress tensor does not simplify to the symmetric velocity gradient;

(b) the total mass density is calculated from the equation $\partial_{t} \varrho+\operatorname{div}(\varrho v)=0$;

(c) the pressure remains partly connected to the other variables by an algebraic formula.

Our main method to approach the PDE problem is a switch of variables in the transport problem as already applied in [5]. Instead of the original variables $\left(\rho_{1}, \ldots, \rho_{N}\right)$ and $\left(p, v_{1}, v_{2}, v_{3}\right)$, we regard $N-1$ linear combinations of the chemical potentials $\left(\mu_{1}, \ldots, \mu_{N}\right)$, the mass density $\varrho$ and the velocity field as main variables. After the transformation we obtain for the new free variables $\left(\varrho, q_{1}, \ldots, q_{N-2}, \zeta, v_{1}, v_{2}, v_{3}\right)$ instead of (2), (3) - the equations (here without external forcing and chemical reactions)

$$
\begin{aligned}
\partial_{t} R_{k}(\varrho, q)+\operatorname{div}\left(R_{k}(\varrho, q) v-\tilde{M}_{k, \ell}(\varrho, q) \nabla q \ell-A_{k}(\varrho, q) \nabla \zeta\right) & =0 \quad \text { for } k=1, \ldots, N-2, \\
\operatorname{div}(v-A(\varrho, q) \cdot \nabla q-d(\varrho, q) \nabla \zeta) & =0 \\
\partial_{t} \varrho+\operatorname{div}(\varrho v) & =0 \\
\partial_{t}(\varrho v)+\operatorname{div}(\varrho v \otimes v-\mathbb{S}(\nabla v))+\nabla P(\varrho, q)+\nabla \zeta & =0 .
\end{aligned}
$$

The nonlinear field $R$ and the function $P$, the vector field $A$, the positive matrix $\tilde{M}$, and the positive coefficient function $d$ will be constructed below, combining certain linear projection operators with the inverse map for the algebraic equations $\mu=$ $\bar{V} p+\nabla_{\rho} k(\rho)$. We are then faced with a nonlinear PDE system of mixed parabolicelliptic-hyperbolic type. All variables are unconstrained, but for the restriction $\varrho_{\min }<$ $\varrho<\varrho_{\max }$ on the total mass density. Here, the constants $0<\varrho_{\min }<\varrho_{\max }<+\infty$ are the thresholds of the total mass for states $\rho_{1}, \ldots, \rho_{N}$ that satisfy the constraint (1):

$$
\begin{array}{r}
\varrho_{\min }:=\min \left\{\sum_{i=1}^{N} \rho_{i}: \rho_{i} \geq 0, \sum_{i=1}^{N} \rho_{i} \bar{V}_{i}=1\right\}=\frac{1}{\max \bar{V}}, \\
\varrho_{\max }:=\max \left\{\sum_{i=1}^{N} \rho_{i}: \rho_{i} \geq 0, \sum_{i=1}^{N} \rho_{i} \bar{V}_{i}=1\right\}=\frac{1}{\min \bar{V}} .
\end{array}
$$

Comparing with the paper [5] on compressible class-one models based on a similar reformulation, we see that the incompressible limit corresponds structurally to the case that one of the relative chemical potentials is subject to an elliptic-instead of a parabolic - equation, and the total mass density is confined to a bounded interval.

For an overview of possible methods to study the transformed PDE system, we refer to our study [5]. We shall follow here the same principal road map, but profound transformations are necessary to deal with the constraint on $\varrho$, since it implies that the nonlinear functions occurring in the transformed system are singular for $\operatorname{dist}\left(\varrho,\left\{\varrho_{\min }, \varrho_{\max }\right\}\right) \rightarrow 0$. The solution operator to the continuity equation, however, does not 'see' these thresholds, which is the source of additional problems when we attempt to linearise. Moreover, we must construct a solution operator for the parabolicelliptic subsystem of general form for $(q, \zeta)$, while the reduced transport problem in 
[5] was purely parabolic. Nontrivial extensions of the method are therefore necessary to deal with the incompressible case.

We shall study the problem in the class proposed in the paper [29] for NavierStokes: $W_{p}^{2,1}$ with $p$ larger than the space dimension for the velocity and $W_{p, \infty}^{1,1}$ for the density. For the variable $q_{1}, \ldots, q_{N-2}$, we also choose the parabolic setting of $W_{p}^{2,1}$. For the elliptic component $\zeta$, we choose the state space $W_{p}^{2,0}$. In these classes, we are able to prove the local existence for strong solutions. In general, we obtain only a short-time well-posedness result, and boundedness in the state space is not sufficient to guarantee that the solution can be extended to a larger time interval. This is due to the constraint $\varrho_{\min }<\varrho<\varrho_{\max }$ : A strong solution with bounded state space norm might break down if the density reaches the thresholds. However, it is to note that for choices of the tensor $M$ reflecting the physically expected behaviour that, in the dilute limit, a diffusion flux is linearly proportional with the mass density of the vanishing species, we are able to show that a sufficiently smooth solution $(p>5)$ bounded in the state space cannot reach the critical values in finite time. Thus, a kind of maximum principle is available for the system.

We shall also prove the global existence under the condition that the initial data are sufficiently near to an equilibrium (stationary) solution. However, since this result relies on stability estimates in the state space, we need to assume higher regularity of the initial data in order to obtain some stability from the continuity equation. Therefore, these solutions exist on arbitrary large time intervals, but do not enjoy the extension property. We shall not make use of the Lagrangian coordinates but employ the approach of controlled growth in time of the solution by means of a priori estimates.

Let us finally mention also the paper [15], devoted to binary mixtures. Starting from different modelling principles in the spirit of [19], the authors derive for $N=2$ a similar PDE system. The variable $q$ does not occur, and the coefficient $d$ is assumed constant. The authors prove for this system the global existence of weak solutions if the singularity of $P(\varrho)$ at the thresholds is sufficiently strong.

The weak solution analysis for the general system is considered in the paper [14].

\subsection{Main results}

We denote $\left.Q=Q_{T}=\Omega \times\right] 0, T$ [ with a bounded domain $\Omega \subset \mathbb{R}^{3}$ and $T>0$ a finite time. We use the standard Sobolev spaces $W^{m, p}(\Omega)$ for $m \in \mathbb{N}$ and $1 \leq p \leq$ $+\infty$, and the Sobolev-Slobodecki spaces $W_{p}^{s}(\Omega)$ for $s>0$ non-integer. If $\Omega$ is a domain of class $\mathcal{C}^{2}$, the spaces $W_{p}^{s}(\partial \Omega)$ are well defined for $0 \leq s \leq 2$.

With a further index $1 \leq r \leq+\infty$, we use the parabolic Lebesgue spaces $L^{p, r}(Q)$ (space index first: $L^{p}(Q)=L^{p, p}(Q)$ ). For $\ell=1,2, \ldots$ and $1 \leq p \leq+\infty$ we introduce the parabolic Sobolev spaces

$$
\begin{aligned}
W_{p}^{2 \ell, \ell}(Q) & :=\left\{u \in L^{p}(Q): D_{t}^{\beta} D_{x}^{\alpha} u \in L^{p}(Q) \forall 1 \leq 2 \beta+|\alpha| \leq 2 \ell\right\}, \\
\|u\|_{W_{p}^{2 \ell, \ell}(Q)} & :=\sum_{0 \leq 2}\left\|D_{t}^{\beta} D_{x}^{\alpha} u\right\|_{L^{p}(Q)},
\end{aligned}
$$


and, with a further index $1 \leq r<\infty$, the spaces

$$
\begin{aligned}
W_{p, r}^{1}(Q)=W_{p, r}^{1,1}(Q) & :=\left\{u \in L^{p, r}(Q): \sum_{0 \leq \beta+|\alpha| \leq \ell} D_{x}^{\alpha} D_{t}^{\beta} u \in L^{p, r}(Q)\right\}, \\
\|u\|_{W_{p, r}^{\ell, \ell}(Q)} & :=\sum_{0 \leq \beta+|\alpha| \leq \ell}\left\|D_{t}^{\beta} D_{x}^{\alpha} u\right\|_{L^{p, r}(Q)} .
\end{aligned}
$$

In these notations, the space integrability index always comes first. For $r=+\infty$, $W_{p, \infty}^{\ell, \ell}(Q)$ denotes the closure of $C^{\ell}(\bar{Q})$ with respect to the norm above and, thus,

$$
W_{p, \infty}^{1,1}(Q):=\left\{u \in L^{p, \infty}(Q): \sum_{0 \leq \beta+|\alpha| \leq 1} D_{x}^{\alpha} D_{t}^{\beta} u \in C\left([0, T] ; L^{p}(\Omega)\right)\right\} .
$$

We also encounter, for $\ell=1,2$ and $1 \leq p<+\infty$,

$$
\begin{aligned}
W_{p}^{\ell, 0}(Q) & :=\left\{u \in L^{p}(Q): \sum_{0 \leq|\alpha| \leq \ell} D_{x}^{\alpha} u \in L^{p}(Q)\right\}, \\
\|u\|_{W_{p}^{\ell, 0}(Q)} & :=\sum_{0 \leq|\alpha| \leq \ell}\left\|D_{x}^{\alpha} u\right\|_{L^{p}(Q) .}
\end{aligned}
$$

We denote by $C(\bar{Q})=C^{0,0}(\bar{Q})$ the space of continuous functions over $\bar{Q}$ and, for $\alpha, \beta \in[0,1]$, the Hölder spaces are defined by $C^{\alpha, \beta}(\bar{Q}):=\{u \in C(\bar{Q})$ : $\left.[u]_{C^{\alpha, \beta}(\bar{Q})}<+\infty\right\}$ with

$$
[u]_{C^{\alpha, \beta}(\bar{Q})}=\sup _{t \in[0, T], x, y \in \Omega} \frac{|u(t, x)-u(t, y)|}{|x-y|^{\alpha}}+\sup _{x \in \Omega, t, s \in[0, T]} \frac{|u(t, x)-u(s, x)|}{|t-s|^{\beta}} .
$$

Some brief remarks on notation:

(1) All Hölder continuity properties are global. For the sake of notation we identify $C^{\alpha, \beta}(Q)$ with $C^{\alpha, \beta}(\bar{Q})$.

(2) Whenever confusion is impossible, we shall also employ for a function $f$ of the variables $x \in \Omega$ and $t \geq 0$ the notations $f_{x}=\nabla f$ for the spatial gradient, and $f_{t}$ for the time derivative.

(3) For maps like $R, \widetilde{M}$ which depend on $\varrho$ and $q$, the derivatives are denoted by $R_{\varrho}, \widetilde{M}_{q}$.

Due to (5), the matrix $M(\rho)$ possesses only $N-1$ positive eigenvalues that moreover might degenerate for vanishing species. The orthogonal projection on the $N-1$ dimensional linear space $\operatorname{span}\left\{1^{N}\right\}^{\perp}$ in $\mathbb{R}^{N}$ is defined via

$$
\mathcal{P}_{\left\{1^{N}\right\}^{\perp}}: \mathbb{R}^{N} \rightarrow\left\{1^{N}\right\}^{\perp}, \quad \mathcal{P}_{\left\{1^{N}\right\}^{\perp}}=\operatorname{Id}_{\mathbb{R}^{N}}-\frac{1}{N} 1^{N} \otimes 1^{N} .
$$

The vector $\bar{V}$ occurring in (1) defines another singular direction in the model preventing parabolicity. We denote by $\mathcal{P}_{\left\{1^{N}, \bar{V}\right\}^{\perp}}$ the orthogonal projection onto the $N-2 \mathrm{dim}$. 
space $\left\{1^{N}, \bar{V}\right\}^{\perp}$. We also introduce the notations

$$
\begin{aligned}
\mathbb{R}_{+}^{N} & :=\left\{\rho=\left(\rho_{1}, \ldots, \rho_{N}\right) \in \mathbb{R}^{N}: \rho_{i}>0 \text { for } i=1, \ldots, N\right\}, \\
\overline{\mathbb{R}}_{+}^{N} & :=\left\{\rho=\left(\rho_{1}, \ldots, \rho_{N}\right) \in \mathbb{R}_{+}^{N}: \rho_{i} \geq 0 \text { for } i=1, \ldots, N\right\}, \\
S_{1} & :=\left\{\rho=\left(\rho_{1}, \ldots, \rho_{N}\right) \in \mathbb{R}_{+}^{N}: \sum_{i=1}^{N} \rho_{i}=1\right\}, \\
S_{\bar{V}} & :=\left\{\rho=\left(\rho_{1}, \ldots, \rho_{N}\right) \in \mathbb{R}_{+}^{N}: \sum_{i=1}^{N} \bar{V}_{i} \rho_{i}=1\right\} .
\end{aligned}
$$

The surface $S_{\bar{V}}$ is the domain of existence for the incompressible state. It is readily seen that $\rho \in S_{\bar{V}}$ implies for the variable $\varrho:=\sum_{i=1}^{N} \rho_{i}$ the inequalities

$$
\varrho_{\min }=\frac{1}{\max _{j=1, \ldots, N} \bar{V}_{j}}<\varrho<\varrho_{\max }=\frac{1}{\min _{j=1, \ldots, N} \bar{V}_{j}} \text { for all } \rho \in S_{\bar{V}} .
$$

Our first main result is devoted to the short-time existence of a strong solution. (In order to avoid notational confusion with the pressure field, the integrability index is called $s$ in the next statements.)

Theorem 1. We fix $s>3$ and $T>0$ and assume that

(a) $\Omega \subset \mathbb{R}^{3}$ is a bounded domain of class $\mathcal{C}^{2}$;

(b) $M: \mathbb{R}_{+}^{N} \rightarrow \mathbb{R}^{N \times N}$ is a mapping of class $C^{2}\left(\mathbb{R}_{+}^{N} ; \mathbb{R}^{N \times N}\right)$ into the positive semidefinite matrices of rank $N-1$ with constant kernel $\operatorname{span}\left\{1^{N}\right\}=\{(1, \ldots, 1)\}$;

(c) $k: \mathbb{R}_{+}^{N} \rightarrow \mathbb{R}$ is of class $C^{3}\left(\mathbb{R}_{+}^{N}\right)$, positively homogeneous, convex in its domain $\mathbb{R}_{+}^{N}$, and $\liminf _{m \rightarrow+\infty}\left|\nabla_{\rho} k\left(y^{m}\right)\right|=+\infty$ for all sequences $\left\{y^{m}\right\} \subset S_{1}$ approaching the relative boundary of $S_{1}$;

(d) $r: \mathbb{R}_{+}^{N} \rightarrow \mathbb{R}^{N}$ is a mapping of class $C^{1}\left(\mathbb{R}_{+}^{N}\right)$ into $\operatorname{span}\left\{1^{N}, \bar{V}\right\}^{\perp}$;

(e) The forcing $b$ satisfies $\mathcal{P}_{\left\{1^{N}\right\}^{\perp}} b \in W_{s}^{1,0}\left(Q_{T} ; \mathbb{R}^{N \times 3}\right)$ and $b-\mathcal{P}_{\left\{1^{N}\right\}^{\perp}} b \in$ $L^{s}\left(Q_{T} ; \mathbb{R}^{N \times 3}\right)$. For simplicity, we assume $v(x) \cdot \mathcal{P}_{\left\{1^{N}\right\}^{\perp}} b(x, t)=0$ for $x \in \partial \Omega$ and $\lambda_{1}$-almost all $\left.t \in\right] 0, T[$.

(f) The initial data $\rho_{1}^{0}, \ldots \rho_{N}^{0}: \bar{\Omega} \rightarrow S_{\bar{V}}$ are positive measurable functions satisfying the following conditions:

- The initial total mass density $\varrho_{0}:=\sum_{i=1}^{N} \rho_{i}^{0}$ is of class $W^{1, s}(\Omega)$;

- The vectorfield $e^{0}:=\partial_{\rho} k\left(\rho_{1}^{0}, \ldots \rho_{N}^{0}\right)$ satisfies $\mathcal{P}_{\left\{1^{N}, \bar{V}\right\}^{\perp}} e^{0} \in W_{s}^{2-2 / s}\left(\Omega ; \mathbb{R}^{N}\right)$;

- The compatibility condition $v(x) \cdot \mathcal{P}_{\left\{1^{N}, \bar{V}\right\}^{\perp}} \nabla e^{0}(x)=0$ is valid in $W_{s}^{1-3 / s}$ $\left(\partial \Omega ; \mathbb{R}^{N}\right)$ in the sense of traces;

(g) The initial velocity $v^{0}$ belongs to $W_{s}^{2-2 / s}\left(\Omega ; \mathbb{R}^{3}\right)$ with $v^{0}=0$ in $W_{s}^{2-3 / s}(\partial \Omega$; $\mathbb{R}^{3}$ ).

Then, there exists $T^{*} \in(0, T]$ such that the problem (2), (3) with closure relations (4), (6), incompressibility constraint (1) and boundary conditions (10), (11), (12), (13) possesses a unique solution ( $\rho, p, v)$ of class

$$
\rho \in W_{s}^{1}\left(Q_{T^{*}} ; S_{\bar{V}}\right), \quad p \in W_{s}^{1,0}\left(Q_{T^{*}}\right), \quad v \in W_{s}^{2,1}\left(Q_{T^{*}} ; \mathbb{R}^{3}\right),
$$


such that $\mu:=p \bar{V}+\partial_{\rho} k(\rho)$ satisfies $\mathcal{P}_{\left\{1^{N}\right\}^{\perp}} \mu \in W_{s}^{2,0}\left(Q_{T^{*}} ; \mathbb{R}^{N}\right)$. The solution can be uniquely extended to a larger time interval whenever the two following conditions are fulfilled:

(i) $\varrho_{\text {min }}<\inf \left\{\varrho(x, t): x \in \bar{\Omega}, t \in\left[0, T^{*}[\}\right.\right.$ and $\sup \{\varrho(x, t): x \in \bar{\Omega}, t \in$ $\left[0, T^{*}[\}<\varrho_{\max }\right.$

(ii) There is $\alpha>0$ such that the quantity

$\left\|\mathcal{P}_{\left\{1^{N}, \bar{V}\right\}^{\perp}} \mu\right\|_{C^{\alpha, \frac{\alpha}{2}\left(Q_{t}\right)}}+\left\|\nabla \mathcal{P}_{\left\{1^{N}, \bar{V}\right\}^{\perp}} \mu\right\|_{L^{\infty, s}\left(Q_{t}\right)}+\|v\|_{L^{z s, s}\left(Q_{t}\right)}+\int_{0}^{t}[\nabla v(\tau)]_{C^{\alpha}(\Omega)} d \tau<\infty$

stays finite as $t \nearrow T^{*}$. Here $z=z(s)$ is defined via $z=3 /(s-2)$ for $3<s<5$, $z>1$ arbitrary for $s=5$ and $z=1$ if $s>5$.

It is to note that the possibility to extend the solution is not-like in the compressible case-reducible to the smoothness criterion (ii). If (i) is failing, even a smooth solution can break down if its total mass density reaches the critical values $\left\{\varrho_{\min }, \varrho_{\max }\right\}$. This singularity plays an important role also in the context of the weak solution analysis (see [14]). However, we provide an important complement for physically motivated choices of the mobility matrix $M$ and of the function $k$. Here the boundedness in the natural state space norm is sufficient to guarantee the extension property.

Theorem 2. In the situation of Theorem 1 we assume, in addition, that $s>5$ and that $k$ is the function defined in (7). We define a matrix $B_{i, j}(\rho):=M_{i, j}(\rho) / \rho_{j}$ for $i, j=1, \ldots, N$, and we assume that there is a continuous function $C=C(|\rho|)$, bounded on compact subsets of $\overline{\mathbb{R}}_{+}^{N} \backslash\{0\}$, such that

$$
\left|B_{i, j}(\rho)\right|+\rho_{k}\left|\partial_{\rho_{k}} B_{i, j}(\rho)\right| \leq C(|\varrho|) \text { for all } i, j, k \in\{1, \ldots, N\} \text { and all } \rho \in \mathbb{R}_{+}^{N} \text {. }
$$

Then the strong solution of Theorem 1 can be extended beyond $T^{*}$ whenever

$$
\lim _{t \nearrow T^{*}}\left\|\mathcal{P}_{\left\{1^{N}, \bar{V}\right\}^{\perp}} \mu\right\|_{W_{s}^{2,1}\left(Q_{t} ; \mathbb{R}^{N}\right)}+\left\|\mathcal{P}_{\left\{1^{N}\right\}^{\perp}} \mu\right\|_{W_{s}^{2,0}\left(Q_{t} ; \mathbb{R}^{N}\right)}+\|v\|_{W_{s}^{2,1}\left(Q_{t} ; \mathbb{R}^{3}\right)}<+\infty .
$$

Our second main result concerns global existence under suitable restrictions on the data. An equilibrium solution for (2), (3) is defined as a vector $\left(\rho_{1}^{\mathrm{eq}}, \ldots, \rho_{N}^{\mathrm{eq}}, p^{\mathrm{eq}}, v_{1}^{\mathrm{eq}}\right.$, $v_{2}^{\mathrm{eq}}, v_{3}^{\mathrm{eq}}$ ) of functions defined in $\Omega$ such that

$$
\rho^{\mathrm{eq}} \in W^{1, s}\left(\Omega ; S_{\bar{V}}\right), \quad p^{\mathrm{eq}} \in W^{1, s}(\Omega), \quad v^{\mathrm{eq}} \in W^{2, s}\left(\Omega ; \mathbb{R}^{3}\right),
$$

the vector $\mu^{\text {eq }}:=p^{\text {eq }} \bar{V}+\nabla_{\rho} k\left(\theta, \rho^{\text {eq }}\right)$ satisfies $\mathcal{P}_{\left\{1^{N}\right\}^{\perp}} \mu^{\text {eq }} \in W^{2, s}\left(\Omega ; \mathbb{R}^{N}\right)$ and the relations

$$
\operatorname{div}\left(\rho_{i}^{\mathrm{eq}} v^{\mathrm{eq}}-\sum_{j=1}^{N} M_{i, j}\left(\rho^{\mathrm{eq}}\right)\left(\nabla \mu_{j}^{\mathrm{eq}}-b^{j}(x)\right)\right)=0 \text { for } i=1, \ldots, N
$$

and

$$
\operatorname{div}\left(\varrho^{\mathrm{eq}} v^{\mathrm{eq}} \otimes v^{\mathrm{eq}}-\mathbb{S}\left(\nabla v^{\mathrm{eq}}\right)\right)+\nabla p^{\mathrm{eq}}=\sum_{i=1}^{N} \rho_{i}^{\mathrm{eq}} b^{i}(x)
$$


are valid in $\Omega$. The boundary conditions are

$$
v^{\mathrm{eq}}=0 \text { and } \nu(x) \cdot M_{i, j}\left(\rho^{\mathrm{eq}}\right)\left(\nabla \mu_{j}^{\mathrm{eq}}-b^{j}(x)\right)=0 \text { on } \partial \Omega .
$$

We show that the problem (2), (3) possesses a unique strong solution on an arbitrary large, but finite time interval if the distance of the initial data to an equilibrium solution is sufficiently small, and if both initial conditions and equilibrium solution are smooth enough.

Theorem 3. We adopt the assumptions of Theorem 1 , but assume also that $r \equiv 0$ and that $b=b(x)$ does not depend on time with $b \in W^{1, s}\left(\Omega ; \mathbb{R}^{N \times 3}\right)$. In addition, we assume that an equilibrium solution $\left(\rho^{e q}, p^{e q}, v^{e q}\right) \in W^{1, s}\left(\Omega ; S_{\bar{V}}\right) \times W^{1, s}(\Omega) \times$ $W^{2, s}\left(\Omega ; \mathbb{R}^{3}\right)$ is given. The associated total mass $\varrho^{e q}:=\sum_{i=1}^{N} \rho_{i}^{e q}$ and the velocity possess the additional regularity $\varrho^{e q} \in W^{2, s}(\Omega)$ and $v^{e q} \in W^{3, s}\left(\Omega ; \mathbb{R}^{3}\right)$. Assume that the initial data satisfies $\varrho^{0} \in W^{2, s}(\Omega)$ and $v^{0} \in W^{2, s}\left(\Omega ; \mathbb{R}^{3}\right)$. Then, for every $0<T<+\infty$, there exists $R_{1}>0$, depending on $T$ and all data in their respective norms, such that under the condition

$$
\left\|\mathcal{P}_{\left\{1^{N}, \bar{V}\right\}^{\perp}}\left(e^{0}-\mu^{e q}\right)\right\|_{W_{s}^{2-\frac{2}{s}}\left(\Omega ; \mathbb{R}^{N}\right)}+\left\|\varrho^{0}-\varrho^{e q}\right\|_{W^{1, s}(\Omega)}+\left\|v^{0}-v^{e q}\right\|_{W_{s}^{2-\frac{2}{s}}\left(\Omega ; \mathbb{R}^{3}\right)} \leq R_{1}
$$

the problem (2), (3) with incompressibility constraint (1), closure relations (4), (6) and the initial and boundary conditions (10), (11), (12), (13) possesses a global unique solution of the same class as in Theorem 1.

\subsection{Road map}

In Sects. 3 and 4 we show how to reformulate the original system such that it becomes easier to tackle via functional analytic methods. The functional setting is discussed in Sect. 5. In Sect. 6, we introduce two ways to linearise the PDE system and reformulate the initial-boundary-value problem as a fixed point problem in the state space. Both fixed point equations exploit the parabolic substructure for the variables $(q, v)$ and treat the linear equations for $(\zeta, \varrho)$ as side conditions. In the first method, used to prove the short-time well posedness, all lower-order nonlinearities are frozen. For the proof of Theorem 3 on small perturbations, a somewhat more elaborated linearisation principle is used in order to exhibit some stability estimates.

The estimates for the linearised principal part of the system are presented in Sect. 7. Here we can rely partly on our work in [5] for the compressible system, but have to discuss the additional problems caused by the presence of an elliptic equation and of a density constraint in the continuity equation. Section 8 shows the self mapping estimate for the first fixed point equation, which yields the well posedness result in Sect. 9. The extension criteria proved for the solution in the same Sect. 9 deserve attention in their own right. The proof of the global well-posedness result for small data, or rather small perturbations, is given in Sect. 10. Finally, some reminder, tools, and purely technical statements are compiled in "Appendix". 


\section{The singular free energy function and its conjugate}

In comparison to the analysis of compressible models in [5], a main specificity of the incompressible model concerns the bulk free energy density and the definition (6) of the chemical potentials. With $k: \mathbb{R}_{+}^{N} \rightarrow \mathbb{R}$ given, we introduce a bulk free energy density defined for $\rho \in \mathbb{R}_{+}^{N}$ of the form

$$
h^{\infty}(\rho):= \begin{cases}k(\rho) & \text { if } \sum_{i=1}^{N} \rho_{i} \bar{V}_{i}=1 \\ +\infty & \text { otherwise. }\end{cases}
$$

The function $h^{\infty}$ is singular, but the subdifferential $\partial h^{\infty}$ is non-empty for every $\rho$ satisfying the incompressiblity constraint $\sum_{i=1}^{N} \rho_{i} \bar{V}_{i}=1$. If the function $k$ is continuously differentiable, it can be shown that $\mu \in \partial h^{\infty}(\rho)$ if and only if there exists $p \in \mathbb{R}$ such that $\mu_{i}=p \bar{V}_{i}+\partial_{\rho_{i}} k(\rho)$ for $i=1, \ldots, N$. It can easily be verified that the number $p$ can be characterised as follows:

$$
p=\sup _{\rho \in \mathbb{R}_{+}^{N}}\left\{\mu \cdot \rho-h^{\infty}(\rho)\right\}=\sup _{\rho \in \mathbb{R}_{+}^{N}, \sum_{i=1}^{N} \rho_{i} \bar{V}_{i}=1}\{\mu \cdot \rho-k(\rho)\}=\left(h^{\infty}\right)^{*}(\mu),
$$

where $\left(h^{\infty}\right)^{*}$ is the convex conjugate of $h^{\infty}$. For systematic discussions and a proof of these elementary statements, we refer to [3].

Our approach essentially relies on the properties of the dual free energy function $f:=\left(h^{\infty}\right)^{*}$ on $\mathbb{R}^{N}$. We shall recall three statements of the paper [3]. Proofs are provided in Appendix, Sect. A for the reader's convenience. In the special case that the gradient of $k$ is explicitly invertible on $S_{1}$ (see (14)), the statements can also be proved by direct algebraic computations yielding in many cases explicit formulae; see Sect. 4 in [14] for a complete characterisation of the example (7).

Lemma 4. We assume that $k: \mathbb{R}_{+}^{N} \rightarrow \mathbb{R}$ is a positively homogeneous convex function of class $C^{3}\left(\mathbb{R}_{+}^{N}\right)$. We moreover assume that the restriction of $k$ to the surface $S_{1}$ is essentially smooth, meaning that $\left|\nabla_{\rho} k\left(y^{m}\right)\right| \rightarrow+\infty$ for sequences $\left\{y^{m}\right\}_{m \in \mathbb{N}} \subset S_{1}$ such that $\min _{i=1, \ldots, N} y_{i}^{m} \rightarrow 0$ as $m \rightarrow+\infty$. For $\mu \in \mathbb{R}^{N}$, we define $f(\mu):=$ $\sup _{\rho \in S_{\bar{V}}}\{\mu \cdot \rho-k(\rho)\}$. Then the function $f$ belongs to $C^{3}\left(\mathbb{R}^{N}\right)$, and $\nabla_{\mu} f$ maps onto $S_{\bar{V}}$.

Lemma 5. We adopt the same assumptions as in Lemma 4. Then

(1) $f(\mu+s \bar{V})=f(\mu)+s$ and $\nabla_{\mu} f(\mu+s \bar{V})=\nabla_{\mu} f(\mu)$ for all $\mu \in \mathbb{R}^{N}$ and all $s \in \mathbb{R}$

(2) The Hessian $D^{2} f(\mu)$ is positive semi-definite for all $\mu \in \mathbb{R}^{N}$, with $\operatorname{ker}\left(D^{2} f(\mu)\right)$ $=\operatorname{span}\{\bar{V}\}$

The next Lemma is a main tool for our reformulation of the PDE system.

Lemma 6. We adopt the assumptions of Lemma 4. If $\mu \in \mathbb{R}^{N}, \rho \in S_{\bar{V}}$ and $p$ are related via (6), then $p=f(\mu)$ and $\rho=\nabla_{\mu} f(\mu)$. 


\section{Change of variables for the incompressible model}

We propose a reformulation of the equations (2), (3) subject to the constitutive equations (4), (6) and to the volume constraint (1) in order to eliminate the positivity constraints on $\rho$, the singularity due to $M 1^{N}=0$ (cf. (5)), and the singularity direction due to the incompressibility (1) - equivalently, the fact that the function $f$, interpreted as the dual of the free energy, is affine in the direction of $\bar{V}\left(D^{2} f \bar{V}=0\right.$, Lemma 5). Like in the investigations in $[5,11,14]$, the idea is to invert the algebraic relations (6) for $\mu, p, \rho$ and to combine this procedure with appropriate linear projections.

\subsection{General ideas}

We choose a basis of $\mathbb{R}^{N}:\left\{\xi^{1}, \ldots, \xi^{N-2}, \xi^{N-1}, \xi^{N}\right\}$ with $\xi^{N}=1^{N}$ and $\xi^{N-1}=$ $\bar{V}$. We then choose $\eta^{1}, \ldots, \eta^{N}$ to be the dual basis, i. e. $\xi^{i} \cdot \eta^{j}=\delta_{j}^{i}$ for $i, j=1, \ldots, N$. We define variables $q^{1}, \ldots, q^{N-2}$ and $\zeta$ via

$$
\begin{aligned}
q_{\ell} & :=\eta^{\ell} \cdot \mu:=\sum_{i=1}^{N} \eta_{i}^{\ell} \mu_{i} \text { for } \ell=1, \ldots, N-2, \\
\zeta\left(=q_{N-1}\right) & :=\eta^{N-1} \cdot \mu=\sum_{i=1}^{N} \eta_{i}^{N-1} \mu_{i} .
\end{aligned}
$$

For $\rho \in \mathbb{R}_{+}^{N}$ such that $\sum_{i=1}^{N} \rho_{i} \bar{V}_{i}=1$, we want to invert the relation $\mu_{i}=\bar{V}_{i} p+$ $\partial_{\rho_{i}} k(\rho)$ for $i=1, \ldots, N$. We exploit the result of Lemma 6 saying that (6) implies $\rho_{i}=$ $\partial_{\mu_{i}} f\left(\mu_{1}, \ldots, \mu_{N}\right)$ for $i=1, \ldots, N$. The vector $\mu$ is then decomposed according to

$$
\mu=\sum_{\ell=1}^{N-2} q^{\ell} \xi^{\ell}+\zeta \bar{V}+\mu \cdot \eta^{N} 1^{N}
$$

into its projection onto $\left\{1^{N}\right\}^{\perp}$, expressed by the variables $q$ and $\zeta$, and its projection on $\operatorname{span}\left\{1^{N}\right\}$.

Next, the last coordinate $\mu \cdot \eta^{N}$ is eliminated using the equation $\varrho=\sum_{i=1}^{N} \rho_{i}=1^{N} \cdot \nabla_{\mu} f\left(\mu_{1}, \ldots, \mu_{N}\right)=1^{N} \cdot \nabla_{\mu} f\left(\sum_{\ell=1}^{N-2} q_{\ell} \xi^{\ell}+\zeta \bar{V}+\left(\mu \cdot \eta^{N}\right) 1^{N}\right)$.

The gradient $\nabla_{\mu} f$ is invariant in the direction $\bar{V}$ (cf. Lemma 5) and, therefore, the variable $\zeta$ decouples from the latter equation, that now reads

$$
\varrho-1^{N} \cdot \nabla_{\mu} f\left(\sum_{\ell=1}^{N-2} q_{\ell} \xi^{\ell}+\left(\mu \cdot \eta^{N}\right) 1^{N}\right)=0 .
$$

This representation is an algebraic equation $F\left(\mu \cdot \eta^{N}, q_{1}, \ldots, q_{N-2}, \varrho\right)=0$. In view of Lemma 5, note that $\partial_{\mu \cdot \eta^{N}} F\left(\mu \cdot \eta^{N}, q_{1}, \ldots, q_{N-2}, \varrho\right)=-D^{2} f(\mu) 1^{N} \cdot 1^{N}<0$, 
due the fact that $1^{N}$ is not parallel to $\bar{V}$. Thus, the last component $\mu \cdot \eta^{N}$ is defined implicitly as a differentiable function of $\varrho$ and $q$. We call this function $\mathscr{M}$ and obtain the equivalent formulation

$$
\begin{aligned}
& \mu=\sum_{\ell=1}^{N-2} q_{\ell} \xi^{\ell}+\zeta \bar{V}+\mathscr{M}\left(\varrho, q_{1}, \ldots, q_{N-2}\right) 1^{N}, \\
& \rho=\nabla_{\mu} f\left(\sum_{\ell=1}^{N-2} q_{\ell} \xi^{\ell}+\mathscr{M}\left(\varrho, q_{1}, \ldots, q_{N-2}\right) 1^{N}\right)=: \mathscr{R}(\varrho, q),
\end{aligned}
$$

where only the total mass density $\varrho$ and the relative chemical potentials $q_{1}, \ldots, q_{N-2}$ and $\zeta$ occur as free variables. Note, moreover, that $\zeta$ and $\rho$ decouple. Similarly, we obtain a representation of the pressure as

$$
\begin{aligned}
p=f(\mu) & =f\left(\sum_{\ell=1}^{N-2} q_{\ell} \xi^{\ell}+\zeta \bar{V}+\mathscr{M}\left(\varrho, q_{1}, \ldots, q_{N-2}\right) 1^{N}\right) \\
& =f\left(\sum_{\ell=1}^{N-2} q_{\ell} \xi^{\ell}+\mathscr{M}\left(\varrho, q_{1}, \ldots, q_{N-2}\right) 1^{N}\right)+\zeta=: P(\varrho, q)+\zeta .
\end{aligned}
$$

All this is summarised in the following Lemma, the proof of which is direct in view of the Lemmas 4 and 6.

Lemma 7. We adopt the assumptions of Theorem 1 for the function $k$. Let $I=$ ]$\varrho_{\min }, \varrho_{\max }\left[\right.$ with $\varrho_{\min }=\min _{i=1, \ldots, N} 1 / \bar{V}_{i}$ and $\varrho_{\max }=\max _{i=1, \ldots, N} 1 / \bar{V}_{i}$. Then there exist a function $\mathscr{M} \in C^{2}\left(I \times \mathbb{R}^{N-2}\right)$ and a field $\mathscr{R} \in C^{2}\left(I \times \mathbb{R}^{N-2} ; S_{\bar{V}}\right)$ such that the equations $\rho=\nabla_{\mu} f(\mu)$ are valid if and only if there are $\varrho \in I, q \in \mathbb{R}^{N-2}$ and $\zeta \in \mathbb{R}$ such that

$$
\sum_{i=1}^{N} \rho_{i}=\varrho, \rho=\mathscr{R}(\varrho, q), \quad \mu=\sum_{j=1}^{N-2} q_{j} \xi^{j}+\zeta \bar{V}+\mathscr{M}(\varrho, q) 1^{N}=: \mu(\varrho, q, \zeta) .
$$

If, moreover, $\mu=\bar{V} p+\partial_{\rho} k(\rho)$ then $p=P(\varrho, q)+\zeta$ with $P \in C^{2}\left(I \times \mathbb{R}^{N-2}\right)$ defined by (21).

In order to deal with the right-hand side (external forcing), we define in the same spirit:

$$
\begin{aligned}
\tilde{b}^{\ell}(x, t) & :=\sum_{i=1}^{N} b^{i}(x, t) \eta_{i}^{\ell} \text { for } \ell=1, \ldots, N-2, \\
\hat{b}(x, t) & :=\sum_{i=1}^{N} b^{i}(x, t) \eta_{i}^{N-1}, \quad \bar{b}(x, t):=\sum_{i=1}^{N} b^{i}(x, t) \eta_{i}^{N} .
\end{aligned}
$$


This allows to express

$$
b^{i}(x, t):=\sum_{\ell=1}^{N-2} \tilde{b}^{\ell}(x, t) \xi_{i}^{\ell}+\hat{b}(x, t) \bar{V}_{i}+\bar{b}(x, t) \text { for } i=1, \ldots, N .
$$

For the reaction terms, we define $\tilde{r}_{\ell}(\varrho, q):=\sum_{i=1}^{N} \xi_{i}^{\ell} r_{i}(\mathscr{R}(\varrho, q))$ for $\ell=1, \ldots, N-$ 2.

4.2. Reformulation of the partial differential equations and of the main theorem

The relation (5) and the equivalence of Lemma 7 show that

$$
\begin{aligned}
J^{i} & =-\sum_{j=1}^{N} M_{i, j}\left(\rho_{1}, \ldots, \rho_{N}\right)\left(\nabla \mu_{j}-b^{j}\right) \\
& =-\sum_{j=1}^{N} M_{i, j}\left(\rho_{1}, \ldots, \rho_{N}\right)\left[\sum_{\ell=1}^{N-2} \xi_{j}^{\ell}\left(\nabla q_{\ell}-\tilde{b}^{\ell}\right)+\bar{V}_{j}(\nabla \zeta-\hat{b})+(\nabla \mathscr{M}(\varrho, q)-\bar{b})\right] \\
& =-\sum_{\ell=1}^{N-2} \sum_{j=1}^{N} M_{i, j}\left(\rho_{1}, \ldots, \rho_{N}\right) \xi_{j}^{\ell}\left(\nabla q_{\ell}-\tilde{b}^{\ell}\right)-\sum_{j=1}^{N} M_{i, j}\left(\rho_{1}, \ldots, \rho_{N}\right) \bar{V}_{j}(\nabla \zeta-\hat{b}) .
\end{aligned}
$$

If we introduce the rectangular projection matrix $\Pi_{j, \ell}=\xi_{j}^{\ell}$ for $\ell=1, \ldots, N-2$ and $j=1, \ldots, N$, then $J=-M \Pi(\nabla q-\tilde{b})-M \bar{V}(\nabla \zeta-\hat{b})$. Thus, we consider equivalently

$$
\begin{gathered}
\partial_{t} \rho+\operatorname{div}(\rho v-M \Pi(\nabla q-\tilde{b})-M \bar{V}(\nabla \zeta-\hat{b}))=r \\
\partial_{t}(\varrho v)+\operatorname{div}(\varrho v \otimes v-\mathbb{S}(\nabla v))+\nabla P(\varrho, q)+\nabla \zeta=\rho \cdot b .
\end{gathered}
$$

In the latter system, we have $\rho=\mathscr{R}(\varrho, q)$ and $\left(\varrho, q_{1}, \ldots, q_{N-2}, \zeta, v_{1}, v_{2}, v_{3}\right)$ are the independent variables. Next, we define for $k=1, \ldots, N-2$ the maps

$R_{k}(\varrho, q):=\sum_{j=1}^{N} \xi_{j}^{k} \rho_{j}=\Pi^{\top} \rho=\sum_{j=1}^{N} \xi_{j}^{k} f_{\mu_{j}}\left(\sum_{\ell=1}^{N-2} q_{\ell} \xi^{\ell}+\mathscr{M}\left(\varrho, q_{1}, \ldots, q_{N-2}\right) 1^{N}\right)$.

Multiplying the mass transfer equations with $\xi_{i}^{k}$, we obtain that $\partial_{t} R_{k}(\varrho, q)+\operatorname{div}\left(R_{k}(\varrho, q) v-\left[\Pi^{\top} M(\rho) \Pi\right]_{k, \ell}\left(\nabla q_{\ell}-\tilde{b}^{\ell}\right)-\left[\Pi^{\top} M(\rho) \bar{V}\right]_{k}(\nabla \zeta-\hat{b})\right)=\tilde{r}_{k}$.

It can be checked easily that the matrix $\Pi^{\top} M(\rho) \Pi \in \mathbb{R}^{(N-2) \times(N-2)}$ is symmetric and strictly positive definite on all states $\rho \in S_{\bar{V}}$. The Jacobian

$$
R_{q}=\Pi^{\top} D^{2} f \Pi-\frac{\Pi^{\top} D^{2} f 1^{N} \otimes \Pi^{\top} D^{2} f 1^{N}}{D^{2} f 1^{N} \cdot 1^{N}},
$$

of size $(N-2) \times(N-2)$ is also strictly positive definite. Indeed, vectors of the form $\Pi a$ in $\mathbb{R}^{N}$ with nonzero $a \in \mathbb{R}^{N-2}$ can by construction never belong to $\operatorname{span}\left\{1^{N}, \bar{V}\right\}$. 
We next multiply the mass balance equations with $\bar{V}_{i}$. Making use of the constraint (1) yields

$$
\operatorname{div}(v-\bar{V} \cdot M(\rho) \Pi(\nabla q-\tilde{b})-\bar{V} \cdot M(\rho) \bar{V}(\nabla \zeta-\hat{b}))=\bar{V} \cdot r=0,
$$

where we use the additional assumption that $r$ maps into $\{\bar{V}\}^{\perp}$. Using that $\rho=$ $\mathscr{R}(\varrho, q)$, we define

$$
\begin{aligned}
\tilde{M}(\varrho, q) & :=\Pi^{\top} M(\mathscr{R}(\varrho, q)) \Pi \in \mathbb{R}^{(N-2) \times(N-2)}, \\
A(\varrho, q) & :=\Pi^{\top} M(\mathscr{R}(\varrho, q)) \bar{V} \in \mathbb{R}^{N-2}, \\
d(\varrho, q) & :=\bar{V} \cdot M(\mathscr{R}(\varrho, q)) \bar{V} .
\end{aligned}
$$

Overall, we get for the variables $\left(\varrho, q_{1}, \ldots, q_{N-2}, \zeta, v\right)$-instead of (2), (3)- the equations

$$
\begin{aligned}
& \partial_{t} R(\varrho, q)+\operatorname{div}(R(\varrho, q) v-\tilde{M}(\varrho, q) \nabla q-A(\varrho, q) \nabla \zeta) \\
& \quad=\tilde{r}(\varrho, q)-\operatorname{div}(\tilde{M}(\varrho, q) \tilde{b}+A(\varrho, q) \hat{b}), \\
& \operatorname{div}(v-A(\varrho, q) \cdot \nabla q-d(\varrho, q) \nabla \zeta)=-\operatorname{div}(A(\varrho, q) \cdot \tilde{b}+d(\varrho, q) \hat{b}), \\
& \partial_{t} \varrho+\operatorname{div}(\varrho v)=0, \\
& \partial_{t}(\varrho v)+\operatorname{div}(\varrho v \otimes v-\mathbb{S}(\nabla v))+\nabla P(\varrho, q)+\nabla \zeta \\
& \quad=R(\varrho, q) \cdot \tilde{b}(x, t)+\hat{b}(x, t)+\varrho \bar{b}(x, t) .
\end{aligned}
$$

The problem $\left(P^{\prime}\right)$ consisting of (25), (26), (27) and (28) for the variables ( $\left.\varrho, q, \zeta, v\right)$ might seem to exhibit more nonlinearities than the original problem for $\rho, p$ and $v$. However, it has the advantage that - up to the restriction on the total mass density $\varrho_{\min }<\varrho<\varrho_{\max }$ - it is completely free of constraints. Furthermore, the differential operator is linear in the variable $\zeta$, which occurs only under spatial differentiation.

Our first aim is now to show that, at least locally in time, the system (25), (26), (27) and (28) for the variables $\left(\varrho, q_{1}, \ldots, q_{N-2}, \zeta, v\right)$ is well posed. We consider initial conditions

$$
q(x, 0)=q_{0}(x), \quad \varrho(x, 0)=\varrho_{0}(x), \quad v(x, 0)=v_{0}(x) \quad \text { for } x \in \Omega .
$$

Due to the preliminary considerations in Sect. 4.1, prescribing these variables is completely equivalent to prescribing initial values for the mass densities $\rho_{i}$ and the velocity. It suffices to define $q_{k}^{0}=\eta^{k} \cdot \partial_{\rho} k\left(\rho^{0}\right)$ for $k=1, \ldots, N-2$.

For simplicity, we consider the linear homogeneous boundary conditions

$$
\begin{aligned}
v & =0 \\
v \cdot \nabla \zeta, \quad v \cdot \nabla q_{k}=0 & \text { on } S_{T}, \\
& \text { on } S_{T} \text { for } k=1, \ldots, N-2 .
\end{aligned}
$$

The conditions (31) and (13) are equivalent, because we assume throughout that the given forcing $b$ satisfies $\nu(x) \cdot \mathcal{P}_{\left\{1^{N}\right\}^{\perp}} b(x, t)=0$ for $x \in \partial \Omega$ (see assumption (e) in the statement of Theorem 1). 
Under the assumptions of Theorem 1 for the function $k$, the coefficient functions $R, \widetilde{M}, A, d$ and $P$ are of class $C^{2}$ in the domain of definitions $I \times \mathbb{R}^{N-2}$ as shown in the Lemma 7. We reformulate the Theorem 1 for the new variables. Since the thermodynamic pressure does not occur explicitly as a variable, we now switch to denoting $p>3$ the integrability exponent (denoted $s$ in the statement 1 ).

Theorem 8. Assume that the coefficient functions $R, \tilde{M}, A, d$ and $P$ are of class $C^{2}$, and $\tilde{r}$ is of class $C^{1}$ in the domain of definition $I \times \mathbb{R}^{N-2}$. Let $\Omega$ be a bounded domain with boundary $\partial \Omega$ of class $\mathcal{C}^{2}$. Suppose that, for some $p>3$, the initial data are of class

$$
q^{0} \in W_{p}^{2-\frac{2}{p}}\left(\Omega ; \mathbb{R}^{N-2}\right), \varrho_{0} \in W^{1, p}(\Omega), v^{0} \in W_{p}^{2-\frac{2}{p}}\left(\Omega ; \mathbb{R}^{3}\right)
$$

satisfying $\varrho_{\min }<\varrho^{0}(x)<\varrho_{\max }$ in $\bar{\Omega}$ and the compatibility conditions $v(x) \cdot \nabla q^{0}(x)=$ 0 and $v^{0}(x)=0$ on $\partial \Omega$. Assume that $\tilde{b} \in W_{p}^{1,0}\left(Q_{T} ; \mathbb{R}^{(N-2) \times 3}\right), \hat{b} \in W_{p}^{1,0}\left(Q_{T} ; \mathbb{R}^{3}\right)$ and $\bar{b} \in L^{p}\left(Q_{T} ; \mathbb{R}^{3}\right)$. Then there is $0<T^{*} \leq T$, depending only on these data, such that the problem (25), (26), (27) and (28) with boundary conditions (29), (30) and (31) is uniquely solvable in the class

$$
(q, \zeta, \varrho, v) \in W_{p}^{2,1}\left(Q_{T^{*}} ; \mathbb{R}^{N-2}\right) \times W_{p}^{2,0}\left(Q_{T^{*}}\right) \times W_{p, \infty}^{1,1}\left(Q_{T^{*}} ; S_{\bar{V}}\right) \times W_{p}^{2,1}\left(Q_{T^{*}} ; \mathbb{R}^{3}\right) .
$$

The solution can be uniquely extended within this class to a larger time interval whenever at least one of the following holds:

(1) $p>5$, the additional conditions of Theorem 2 for $M$ and $k$ are valid, and the state space norm stays finite as $t \rightarrow T^{*}$

(2) The two following conditions are valid as $t \nearrow T^{*}$

$-\varrho_{\min }<\varrho(x, t)<\varrho_{\max }$ for all $x \in \bar{\Omega}$;

$-\|q\|_{C^{\alpha, \frac{\alpha}{2}}\left(Q_{t}\right)}+\|\nabla q\|_{L^{\infty, p}\left(Q_{t}\right)}+\|v\|_{L^{z} p, p\left(Q_{t}\right)}+\int_{0}^{t}[\nabla v(\tau)]_{C^{\alpha}(\Omega)} d \tau<+\infty$, with $\alpha>0$ and $z=z(p)$ defined by Theorem 1 .

\section{Functional analytic approach}

For functions $q_{1}, \ldots, q_{N-2}, \zeta, \varrho$ and $v_{1}, v_{2}, v_{3}$ defined in $\bar{\Omega} \times[0, T]$, we introduce

$$
\begin{aligned}
& \mathscr{A}(q, \zeta, \varrho, v)=\left(\mathscr{A}^{1}(q, \zeta, \varrho, v), \mathscr{A}^{2}(q, \zeta, \varrho, v), \mathscr{A}^{3}(\varrho, v), \mathscr{A}^{4}(q, \zeta, \varrho, v)\right), \\
& \mathscr{A}^{1}(q, \zeta, \varrho, v):= \partial_{t} R(\varrho, q)+\operatorname{div}(R(\varrho, q) v)-\operatorname{div}(\tilde{M}(\varrho, q)(\nabla q-\tilde{b}) \\
&\quad+A(\varrho, q)(\nabla \zeta-\hat{b}))-\tilde{r}(\varrho, q), \\
& \mathscr{A}^{2}(q, \zeta, \varrho, v):= \operatorname{div}(v-d(\varrho, q)(\nabla \zeta-\hat{b})-A(\varrho, q) \cdot(\nabla q-\tilde{b})), \\
& \mathscr{A}^{3}(\varrho, v):= \partial_{t} \varrho+\operatorname{div}(\varrho v), \\
& \mathscr{A}^{4}(q, \zeta, \varrho, v):=\varrho\left(\partial_{t} v+(v \cdot \nabla) v\right)-\operatorname{div} \mathbb{S}(\nabla v)+\nabla P(\varrho, q)+\nabla \zeta-R(\varrho, q) \cdot \tilde{b}-\hat{b}-\varrho \bar{b} .
\end{aligned}
$$

Recall that $\tilde{b}, \hat{b}$ and $\bar{b}$ are given coefficients. 
To get rid of the highest-order coupling in the time derivative of $\varrho$, we shall employ the same approach as in [5], which is sketched below. Consider a solution $u=(q, \zeta, \varrho, v)$ to $\mathscr{A}(u)=0$. Computing time derivatives in the equation $\mathscr{A}^{1}(u)=0$, we obtain that

$$
\begin{aligned}
& \left.R_{\varrho}\left(\partial_{t} \varrho+v \cdot \nabla \varrho\right)+\sum_{j=1}^{N-1} R_{q_{j}}\left(\partial_{t} q_{j}+v \cdot \nabla q_{j}\right)+R \operatorname{div} v-\operatorname{div}(\tilde{M} \nabla q)+A \nabla \zeta\right) \\
& =-\operatorname{div}(\tilde{M} \tilde{b}+A \hat{b})+\tilde{r} .
\end{aligned}
$$

Here the nonlinear functions $R, R_{\varrho}, R_{q}, A$ and $\tilde{M}, \tilde{r}$ etc. are evaluated at $(\varrho, q)$. Under the side-condition $\mathscr{A}^{3}(\varrho, v)=0$, the equation $\mathscr{A}^{1}(u)=0$ is equivalent to

$$
\begin{aligned}
& R_{q}(\varrho, q) \partial_{t} q-\operatorname{div}(\tilde{M}(\varrho, q) \nabla q+A(\varrho, q) \nabla \zeta)=\left(R_{\varrho}(\varrho, q) \varrho\right. \\
& \quad-R(\varrho, q)) \operatorname{div} v-R_{q}(\varrho, q) v \cdot \nabla q-\operatorname{div}(\tilde{M}(\varrho, q) \tilde{b}+A(\varrho, q) \hat{b})+\tilde{r}(\varrho, q) .
\end{aligned}
$$

We introduce $\tilde{\mathscr{A}}(q, \zeta, \varrho, v):=\left(\tilde{\mathscr{A}}^{1}(q, \zeta, \varrho, v), \mathscr{A}^{2}(q, \zeta, \varrho, v), \mathscr{A}^{3}(\varrho, v)\right.$, $\left.\mathscr{A}^{4}(q, \zeta, \varrho, v)\right)$, the first component being the differential operator defined by (32). Clearly, $\mathscr{A}(u)=0$ if and only if $\tilde{\mathscr{A}}(u)=0$.

The functional setting was introduced in Sect. 2.2. Similar spaces were used in [5] to study the compressible system and, in order to save room, we shall refer to this paper for the trace and embedding theorems needed in the present analysis. For $p>3$ and $\alpha:=1 / 2+3 /(2 p)$, we recall the interpolation inequality (see [26], Theorem 1)

$$
\|\nabla f\|_{L^{\infty}(\Omega)} \leq C_{1}\left\|D^{2} f\right\|_{L^{p}(\Omega)}^{\alpha}\|f\|_{L^{p}(\Omega)}^{1-\alpha}+C_{2}\|f\|_{L^{p}(\Omega)},
$$

valid for any function $f$ in $W^{2, p}(\Omega)$, with certain constants $C_{1}, C_{2}$ depending only on $\Omega$. We consider the operator $(q, \zeta, \varrho, v) \mapsto \mathscr{A}(q, \zeta, \varrho, v)$ acting on

$$
\mathcal{X}_{T}:=W_{p}^{2,1}\left(Q_{T} ; \mathbb{R}^{N-2}\right) \times W_{p}^{2,0}\left(Q_{T}\right) \times W_{p, \infty}^{1,1}\left(Q_{T}\right) \times W_{p}^{2,1}\left(Q_{T} ; \mathbb{R}^{3}\right) .
$$

The natural trace space at time zero is denoted $\operatorname{Tr}_{\Omega \times\{0\}} \mathcal{X}_{T}$. The functional setting does not allow to introduce traces for the variable $\zeta$. Therefore, $u(0) \in \operatorname{Tr}_{\Omega \times\{0\}} \mathcal{X}_{T}$ means that $(q(0), \varrho(0), v(0)) \in W_{p}^{2-2 / p}\left(\Omega ; \mathbb{R}^{N-2}\right) \times W^{1, p}(\Omega) \times W_{p}^{2-2 / p}\left(\Omega ; \mathbb{R}^{3}\right)$. We denote by ${ }_{0} \mathcal{X}_{T}$ the space of functions fulfilling zero initial conditions. This only makes sense, of course, for the variables having traces at $\Omega \times\{0\}$. Thus

$$
{ }_{0} \mathcal{X}_{T}:=\left\{\bar{u}=(r, \chi, \sigma, w) \in \mathcal{X}_{T}: r(0)=0, \sigma(0)=0, w(0)=0\right\} .
$$

Since the coefficients of $\mathscr{A}$ are defined only if $\varrho$ has range in $I$, the domain of the operator is contained in the subset

$$
\mathcal{X}_{T, I}:=W_{p}^{2,1}\left(Q_{T} ; \mathbb{R}^{N-2}\right) \times W_{p}^{2,0}\left(Q_{T}\right) \times W_{p, \infty}^{1,1}\left(Q_{T} ; I\right) \times W_{p}^{2,1}\left(Q_{T} ; \mathbb{R}^{3}\right) .
$$


We shall moreover make use of a reduced state space containing only the parabolic components $(q, v)$, namely

$$
\mathcal{Y}_{T}:=W_{p}^{2,1}\left(Q_{T} ; \mathbb{R}^{N-2}\right) \times W_{p}^{2,1}\left(Q_{T} ; \mathbb{R}^{3}\right)
$$

The operator $\mathscr{A}$ is the composition of differentiation, multiplication and Nemicki operators. Therefore, the properties of the coefficients $R, \tilde{M}$ etc. allow to show that $\mathscr{A}$ is continuous and bounded from $\mathcal{X}_{T, I}$ into

$$
\mathcal{Z}_{T}=L^{p}\left(Q_{T} ; \mathbb{R}^{N-2}\right) \times L^{p}\left(Q_{T}\right) \times L^{p, \infty}\left(Q_{T}\right) \times L^{p}\left(Q_{T} ; \mathbb{R}^{3}\right) .
$$

Since the coefficients $R, \tilde{M}, A, d$ and $P$ are twice continuously differentiable in their domain of definition $I \times \mathbb{R}^{N-2}$, the operator $\mathscr{A}$ is even continuously differentiable at every point of $\mathcal{X}_{T, I}$. We spare the proof of these rather obvious statements.

\section{Linearisation and reformulation as a fixed-point equation}

We shall present two different manners to linearise the equation $\tilde{\mathscr{A}}(u)=0$ for $u \in \mathcal{X}_{T}$ with initial condition $u(0)=u_{0}$ in $\operatorname{Tr}_{\Omega \times\{0\}} \mathcal{X}_{T}$. They correspond to the two main Theorems 1,3 , respectively. In both cases, we start considering the problem to find $u=(q, \zeta, \varrho, v) \in \mathcal{X}_{T, I}$ such that $\tilde{\mathscr{A}}(u)=0$ and $u(0)=u_{0}$, which after permuting rows, possesses the following structure

$$
\begin{aligned}
\partial_{t} \varrho+\operatorname{div}(\varrho v) & =0, \\
R_{q}(\varrho, q) \partial_{t} q-\operatorname{div}(\tilde{M}(\varrho, q) \nabla q+A(\varrho, q) \nabla \zeta) & =g(x, t, q, \varrho, v, \nabla q, \nabla \varrho, \nabla v), \\
-\operatorname{div}(d(\varrho, q) \nabla \zeta+A(\varrho, q) \nabla q-v) & =-\operatorname{div} h(x, t, \varrho, q), \\
\varrho \partial_{t} v-\operatorname{div} \mathbb{S}(\nabla v)+\nabla \zeta & =f(x, t, q, \varrho, v, \nabla q, \nabla \varrho, \nabla v) .
\end{aligned}
$$

The functions $g, h$ and $f$ stand for the following expressions:

$$
\begin{aligned}
g:= & \left(R_{\varrho}(\varrho, q) \varrho-R(\varrho, q)\right) \operatorname{div} v-R_{q}(\varrho, q) v \cdot \nabla q-\operatorname{div}(\tilde{M}(\varrho, q) \tilde{b}+A(\varrho, q) \hat{b}) \\
& \quad+\tilde{r}(\varrho, q) \\
h:= & d(\varrho, q) \hat{b}+A(\varrho, q) \tilde{b} \\
f:= & -P_{\varrho}(\varrho, q) \nabla \varrho-P_{q}(\varrho, q) \nabla q-\varrho(v \cdot \nabla) v+R(\varrho, q) \cdot \tilde{b}+\hat{b}+\varrho \bar{b}
\end{aligned}
$$

These expressions are independent on the component $\zeta$. We can regard $g, h$ and $f$ as functions of $x, t$ and of the vectors $u$ and $D_{x} u$ and write $g\left(x, t, u, D_{x} u\right)$. 


\subsection{The first fixed-point equation}

For $\left(q^{*}, v^{*}\right)$ given in $W_{p}^{2,1}\left(Q_{T} ; \mathbb{R}^{N-2}\right) \times W_{p}^{2,1}\left(Q_{T} ; \mathbb{R}^{3}\right)$ and for unknowns $u=$ $(q, \zeta, \varrho, v)$, we consider the following system of equations

$$
\begin{aligned}
\partial_{t} \varrho+\operatorname{div}\left(\varrho v^{*}\right) & =0, \\
R_{q}\left(\varrho, q^{*}\right) \partial_{t} q-\operatorname{div}\left(\tilde{M}\left(\varrho, q^{*}\right) \nabla q+A\left(\varrho, q^{*}\right) \nabla \zeta\right) & =g\left(x, t, q^{*}, \varrho, v^{*}, \nabla q^{*}, \nabla \varrho, \nabla v^{*}\right), \\
-\operatorname{div}\left(d\left(\varrho, q^{*}\right) \nabla \zeta+A\left(\varrho, q^{*}\right) \nabla q\right) & =-\operatorname{div}\left(v^{*}+h\left(x, t, q^{*}, \varrho\right)\right), \\
\varrho \partial_{t} v-\operatorname{div} \mathbb{S}(\nabla v)+\nabla \zeta & =f\left(x, t, q^{*}, \varrho, v^{*}, \nabla q^{*}, \nabla \varrho, \nabla v^{*}\right),
\end{aligned}
$$

together with the initial conditions (29) and the homogeneous boundary conditions (30), (31). Note that the continuity equation can be solved independently for $\varrho$. Once $\varrho$ is given, we solve the linear parabolic-elliptic system (47), (48) for $q$ and $\zeta$. Here we must be careful, since the coefficients of this system are only defined as long as $\varrho(x, t)$ takes values in $I$. Thus, the solution $(q, \zeta)$ might exist only on a shorter time interval. We can solve the problem (49), which is linear in $v$, under the same restriction.

We will show that the solution map $\left(q^{*}, v^{*}\right) \mapsto(q, v)$, denoted $\mathcal{T}$, is well defined from $\mathcal{Y}_{T}$ into itself for $T$ fixed and suitably small. The solutions are unique in the class $\mathcal{Y}_{T}$. Clearly, a fixed point of $\mathcal{T}$ is a solution to $\tilde{\mathscr{A}}(q, \zeta, \varrho, v)=0$.

\subsection{The second fixed-point equation}

Here we construct the fixed-point map comparing the solutions to a given reference vector $\left(\hat{q}^{0}, \hat{v}^{0}\right) \in \mathcal{Y}_{T}$ that extends the initial data. We assume that $\hat{q}^{0}$ and $\hat{v}^{0}$ satisfy the initial compatibility conditions. In order to find an extension for $\varrho_{0} \in W^{1, p}(\Omega)$, we solve the problem

$$
\partial_{t} \hat{\varrho}_{0}+\operatorname{div}\left(\hat{\varrho}_{0} \hat{v}^{0}\right)=0, \quad \hat{\varrho}_{0}(0)=\varrho_{0} .
$$

For this problem, Theorem 2 of [29] establishes unique solvability in $W_{p, \infty}^{1,1}\left(Q_{T}\right)$ and, in particular, the strict positivity $\hat{\varrho}_{0} \geq c_{0}\left(\Omega,\left\|\hat{v}^{0}\right\|_{W_{p}^{2,1}\left(Q_{T} ; \mathbb{R}^{3}\right)}\right) \inf _{x \in \Omega} \varrho_{0}(x)$.

We find the extension $\hat{\zeta}^{0}$ by solving, for all values of $t$ such that the coefficients $\tilde{b}(t)$ and $\hat{b}(t)$ are defined, the elliptic problem

$$
-\operatorname{div}\left(d\left(\hat{\varrho}^{0}, \hat{q}^{0}\right) \nabla \hat{\zeta}^{0}\right)=\operatorname{div}\left(-\hat{v}^{0}-d\left(\hat{\varrho}^{0}, \hat{q}^{0}\right) \hat{b}(t)+A\left(\hat{\varrho}^{0}, \hat{q}^{0}\right) \nabla\left(\hat{q}^{0}-\tilde{b}(t)\right)\right),
$$

with homogeneous Neumann boundary conditions and zero mean-value side-condition.

Consider a solution $u=(q, \zeta, \varrho, v) \in \mathcal{X}_{T}$ to $\widetilde{\mathscr{A}}(u)=0$. We introduce the differences $r:=q-\hat{q}^{0}, \chi=\zeta-\hat{\zeta}^{0}, w:=v-\hat{v}^{0}$ and $\sigma:=\varrho-\hat{\varrho}^{0}$, and their vector $\bar{u}:=(r, \chi, \sigma, w)$. Clearly, $\bar{u}$ belongs to the space ${ }_{0} \mathcal{X}_{T}$ of homogeneous initial conditions. Recall that this does not imply a trace condition for $\chi$, cp. (35). The 
equations $\tilde{\mathscr{A}}(u)=0$ mean, equivalently, that $\tilde{\mathscr{A}}\left(\hat{u}^{0}+\bar{u}\right)=0$. The vector $\bar{u}=$ $(r, \chi, \sigma, w)$ satisfies

$$
\begin{aligned}
R_{q} \partial_{t} r-\operatorname{div}(\tilde{M} \nabla r+A \nabla \chi) & =g^{1}:=g-R_{q} \partial_{t} \hat{q}^{0}+\operatorname{div}\left(\tilde{M} \nabla \hat{q}^{0}+A \nabla \hat{\zeta}^{0}\right), \\
-\operatorname{div}(d \nabla \chi+A \nabla r-w) & =-\operatorname{div} h^{1}:=-\operatorname{div}\left(h+\hat{v}^{0}-d \nabla \hat{\zeta}^{0}-A \nabla \hat{q}^{0}\right) \\
\partial_{t} \sigma+\operatorname{div}(\sigma v) & =-\operatorname{div}\left(\hat{\varrho}_{0} w\right), \\
\varrho \partial_{t} w-\operatorname{div} \mathbb{S}(\nabla w)+\nabla \chi & =f^{1}=: f-\varrho \partial_{t} \hat{v}^{0}+\operatorname{div} \mathbb{S}\left(\nabla \hat{v}^{0}\right)-\nabla \hat{\zeta}^{0}
\end{aligned}
$$

Herein, all nonlinear coefficients $R, R_{q}$, etc. are evaluated at $(\varrho, q)$, while $g, h$ and $f$ correspond to (43), (44) and (45).

We next want to construct a fixed-point map to solve (52), (53), (54), (55) by linearising $g^{1}, h^{1}$ and $f^{1}$ defined in (52), (53) and (55). First, we expand as follows:

$$
\begin{aligned}
g= & g\left(x, t, u^{*}, D_{x} u^{*}\right)+\int_{0}^{1}\left\{\left(g_{q}\right)^{\theta}\left(q-q^{*}\right)+\left(g_{\varrho}\right)^{\theta}\left(\varrho-\varrho^{*}\right)+\left(g_{v}\right)^{\theta}\left(v-v^{*}\right)\right. \\
& \left.+\left(g_{q_{x}}\right)^{\theta} \cdot\left(q_{x}-q_{x}^{*}\right)+\left(g_{\varrho_{x}}\right)^{\theta}\left(\varrho_{x}-\varrho_{x}^{*}\right)+\left(g_{v_{x}}\right)^{\theta} \cdot\left(v_{x}-v_{x}^{*}\right)\right\} d \theta .
\end{aligned}
$$

Here, $(\cdot)^{\theta}$ applied to a function of $x, t, u$ and $D_{x}^{1} u$ stands for the evaluation at $\left(x, t,(1-\theta) u^{*}+\theta u,(1-\theta) D_{x} u^{*}+\theta D_{x} u\right)$. In short, in order to avoid the integral and the parameter $\theta$, we write

$$
\begin{aligned}
g= & g\left(x, t, u^{*}, D_{x} u^{*}\right)+g_{q}\left(u, u^{*}\right)\left(q-q^{*}\right)+g_{\varrho}\left(u, u^{*}\right)\left(\varrho-\varrho^{*}\right)+g_{v}\left(u, u^{*}\right)\left(v-v^{*}\right) \\
& +g_{q_{x}}\left(u, u^{*}\right) \cdot\left(q_{x}-q_{x}^{*}\right)+g_{\varrho_{x}}\left(u, u^{*}\right)\left(\varrho_{x}-\varrho_{x}^{*}\right)+g_{v_{x}}\left(u, u^{*}\right) \cdot\left(v_{x}-v_{x}^{*}\right) \\
= & : g\left(x, t, u^{*}, D_{x} u^{*}\right)+g^{\prime}\left(u, u^{*}\right)\left(u-u^{*}\right) .
\end{aligned}
$$

Obviously, the latter expressions make sense only if $u, u^{*}$ both belong to $\mathcal{X}_{T, I}$, in which case the entire convex hull $\left\{\theta u+(1-\theta) u^{*}: \theta \in[0,1]\right\}$ is in $\mathcal{X}_{T, I}$. Following the same scheme as for (57), we write in short

$$
\begin{aligned}
g^{1}= & g^{1}\left(x, t, \hat{q}^{0}, \hat{\varrho}^{0}, \hat{v}^{0}, \hat{q}_{x}^{0}, \hat{\varrho}_{x}^{0}, \hat{v}_{x}^{0}\right)+g_{q}^{1}\left(u, \hat{u}^{0}\right) r+g_{\varrho}^{1}\left(u, \hat{u}^{0}\right) \sigma+g_{v}^{1}\left(u, \hat{u}^{0}\right) w \\
& +g_{q_{x}}^{1}\left(u, \hat{u}^{0}\right) r_{x}+g_{\varrho_{x}}^{1}\left(u, \hat{u}^{0}\right) \sigma_{x}+g_{v_{x}}^{1}\left(u, \hat{u}^{0}\right) w_{x} \\
= & : \hat{g}^{0}+\left(g^{1}\right)^{\prime}\left(u, \hat{u}^{0}\right) \bar{u} .
\end{aligned}
$$

Similar expressions are obtained for $h^{1}$ and $f^{1}$. In the case of $h^{1}$, note however that $\operatorname{div} \hat{h}^{0}=\operatorname{div}\left(h^{1}\left(x, t, \hat{q}^{0}, \hat{\varrho}^{0}, \hat{v}^{0}, \hat{q}_{x}^{0}, \hat{\varrho}_{x}^{0}, \hat{v}_{x}^{0}\right)=0\right.$ due to the construction (51) of $\hat{\zeta}^{0}$.

Now we construct the fixed-point map to solve (52), (53), (54) and (55). For a given vector $\left(r^{*}, w^{*}\right) \in{ }_{0} \mathcal{Y}_{T}$, we define $q^{*}:=\hat{q}^{0}+r^{*}$ and $v^{*}:=\hat{v}^{0}+w^{*}$. Then we define $\varrho^{*}$ to be the unique solution to

$$
\partial_{t} \varrho^{*}+\operatorname{div}\left(\varrho^{*} v^{*}\right)=0, \quad \varrho^{*}(x, 0)=\varrho^{0}(x) .
$$


We thus write $\varrho^{*}:=\mathscr{C}\left(v^{*}\right)$ where $\mathscr{C}$ is the solution operator to the continuity equation with initial data $\varrho_{0}$. We employ the abbreviation

$$
u^{*}:=\left(q^{*}, 1, \varrho^{*}, v^{*}\right)=\left(q^{*}, 1, \mathscr{C}\left(v^{*}\right), v^{*}\right) \in \mathcal{X}_{T} .
$$

For $\bar{u}:=(r, \chi, \sigma, w)$, we next consider the linear problem

$$
\begin{aligned}
R_{q}^{*} \partial_{t} r-\operatorname{div}\left(\tilde{M}^{*} \nabla r+A^{*} \nabla \chi\right) & =\hat{g}^{0}+\left(g^{1}\right)^{\prime}\left(u^{*}, \hat{u}^{0}\right) \bar{u}, \\
-\operatorname{div}\left(d^{*} \nabla \chi+A^{*} \nabla r-w\right) & =-\operatorname{div}\left(\left(h^{1}\right)^{\prime}\left(u^{*}, \hat{u}^{0}\right) \bar{u}\right), \\
\partial_{t} \sigma+\operatorname{div}\left(\sigma v^{*}\right) & =-\operatorname{div}\left(\hat{\varrho}_{0} w\right), \\
\mathscr{C}\left(v^{*}\right) \partial_{t} w-\operatorname{div} \mathbb{S}(\nabla w)+\nabla \chi & =\hat{f}^{0}+\left(f^{1}\right)^{\prime}\left(u^{*}, \hat{u}^{0}\right) \bar{u},
\end{aligned}
$$

with the boundary conditions $v \cdot \nabla r=0=v \cdot \nabla \chi$ on $S_{T}$ and $w=0$ on $S_{T}$, and with zero initial conditions for $r, \sigma$ and $w$. The superscript $*$ on a coefficient means evaluation at $\left(\mathscr{C}\left(v^{*}\right), q^{*}\right)$.

We will show that the solution map $\mathcal{T}^{1}:\left(r^{*}, w^{*}\right) \mapsto(r, w)$ is well defined from ${ }_{0} \mathcal{Y}_{T}$ into itself for $T>0$ arbitrary, provided that the distance of the initial data to an equilibrium solution is sufficiently small. As to the latter restriction, note that the expressions $\left(g^{1}\right)^{\prime}\left(u^{*}, \hat{u}^{0}\right)$ make sense only if the density components in both $u^{*}$ and $\hat{u}^{0}$ map into the interior of the critical interval, which cannot be expected globally for the solutions to (50) and (59). If $\bar{u}=(r, w)$ is a fixed point of $\mathcal{T}^{1}$, then we can show that $u:=\hat{u}^{0}+\bar{u}$ is a solution to $\tilde{\mathscr{A}}(u)=0$. This is verified exactly as in [5], Remark 6.1 .

\subsection{The self-mapping property}

Assume that the map $\mathcal{T}:\left(q^{*}, v^{*}\right) \mapsto(q, v)$ via the solution to (46), (47), (48), (49) is well defined in $\mathcal{Y}_{T}$, with image in $\mathcal{Y}_{\tilde{T}}$ for some $\tilde{T}=\tilde{T}\left(q^{*}, v^{*}\right)>0$. Then, we want to show that $\mathcal{T}$ maps some closed bounded set of $\mathcal{Y}_{T_{0}}$ into itself for a fixed $T_{0}>0$. Here, a major change occurs in comparison to the compressible case, since we do not expect that the linearised map $\mathcal{T}$ produces a solution defined globally up to $T$. This is due to the constraint $\varrho \in] \varrho_{\min }, \varrho_{\max }[$ which can by nature be enforced only locally for solutions to the continuity equation (46).

We shall rely on continuous estimates expressing the controlled growth of the solution in time. We will show that there is a parameter $a_{0}$ depending on the distance of the initial density to the singular values $\left\{\varrho_{\min }, \varrho_{\max }\right\}$ such that, whenever $t>0$ satisfies $t^{1-\frac{1}{p}}\left\|\left(q^{*}, v^{*}\right)\right\| \mathcal{Y}_{t}<a_{0}$, the pair $(q, v)=\mathcal{T}\left(q^{*}, v^{*}\right)$ is well defined in $\mathcal{Y}_{t}$ and satisfies the estimate

$$
\begin{aligned}
& \|(q, v)\|_{W_{p}^{2,1}\left(Q_{t} ; \mathbb{R}^{N-2}\right) \times W_{p}^{2,1}\left(Q_{t} ; \mathbb{R}^{3}\right)} \\
& \quad \leq \Psi\left(t, R_{0},\left\|\left(q^{*}, v^{*}\right)\right\|_{W_{p}^{2,1}\left(Q_{t} ; \mathbb{R}^{N-2}\right) \times W_{p}^{2,1}\left(Q_{t} ; \mathbb{R}^{3}\right)}\right) .
\end{aligned}
$$

Here $R_{0}$ stands for the magnitude of the initial data $q^{0}, \varrho_{0}$ and $v^{0}$, and of the external forces $b$ in their respective norms. The function $\Psi$ is continuous, increasing in all 
arguments, and finite for $t^{1-\frac{1}{p}}\left\|\left(q^{*}, v^{*}\right)\right\|_{\mathcal{Y}_{t}}<a_{0}$. Hence we obtain a self mapping property with the help of the following Lemma.

Lemma 9. Suppose that $R_{0}$ is fixed. Suppose that there is $a_{0}>0$ such that the inequality (65) is valid with a continuous function $\Psi=\Psi\left(t, R_{0}, \eta\right)$ satisfying the properties:

$-\Psi\left(\cdot, R_{0}, \cdot\right)$ is finite for all $t \geq 0$ and $\eta \geq 0$ satisfying $t^{1-\frac{1}{p}} \eta<a_{0}$;

- $t \mapsto \Psi\left(t, R_{0}, \eta\right)$ is nondecreasing for all $0 \leq \eta$, and $\eta \mapsto \Psi\left(t, R_{0}, \eta\right)$ is nondecreasing for all $t$ as long as $t^{1-\frac{1}{p}} \eta<a_{0}$;

- The value of $\Psi\left(0, R_{0}, \eta\right)=\Psi^{0}\left(R_{0}\right)>0$ is independent on $\eta$.

Then there is $t_{0}=t_{0}\left(R_{0}\right)>0$ such that the map $\mathcal{T}\left(q^{*}, v^{*}\right):=(q, v)$ maps a ball of $\mathcal{Y}_{t_{0}}$ into itself.

Proof. In $\left\{(t, \eta) \in\left[\mathbb{R}_{+}\right]^{2}: t^{1-1 / p} \eta<a_{0}\right\}$, the function $(t, \eta) \mapsto \Psi\left(t, R_{0}, \eta\right)$ is continuous and finite. Then, there is a first $t_{0}>0$ depending only on $R_{0}$ such that

$$
\left\{\eta>0: \Psi\left(t_{0}, R_{0}, \eta\right) \leq \eta \text { and } \eta<a_{0} t_{0}^{\frac{1}{p}-1}\right\} \neq \emptyset .
$$

Otherwise, for all $t>0$ and $\eta<a_{0} t^{1 / p-1}$, we would have that $\Psi\left(t, R_{0}, \eta\right)>\eta$. Thus, $\Psi\left(0, R_{0}, \eta\right)=\lim _{t \rightarrow 0} \Psi\left(t, R_{0}, \eta\right) \geq \eta$ for all $\eta>0$. Since $\Psi\left(0, R_{0}, \eta\right)=$ $\Psi^{0}\left(R_{0}\right)$ is strictly positive, every choice of $\eta>\Psi^{0}\left(R_{0}\right)$ then yields a contradiction.

We can further show that

$$
0<\eta_{0}:=\inf \left\{\eta>0: \Psi\left(t_{0}, R_{0}, \eta\right) \leq \eta \text { and } \eta<a_{0} t_{0}^{\frac{1}{p}-1}\right\} .
$$

Otherwise, there are positive $\left\{\eta_{k}\right\}_{k \in \mathbb{N}}, \eta_{k} \searrow 0$, such that $\Psi\left(t_{0}, R_{0}, \eta_{k}\right) \leq \eta_{k}$ for all $k$. Then $0 \geq \lim _{k \rightarrow \infty} \Psi\left(t_{0}, R_{0}, \eta_{k}\right)=\Psi\left(t_{0}, R_{0}, 0\right)$. Since $\Psi\left(t_{0}, R_{0}, 0\right) \geq$ $\Psi\left(0, R_{0}, 0\right)=\Psi^{0}\left(R_{0}\right)>0$, this is again a contradiction.

Consider $M:=\left\{\left(q^{*}, v^{*}\right) \in \mathcal{Y}_{t_{0}}:\left\|\left(q^{*}, v^{*}\right)\right\|_{\mathcal{Y}_{0}} \leq \eta_{0}\right\}$. Since $\eta_{0}<a_{0} t_{0}^{1 / p-1}$, it follows that $t_{0}^{1-1 / p}\left\|\left(q^{*}, v^{*}\right)\right\|_{\mathcal{Y}_{0}}<a_{0}$. The inequality (65) is valid by assumption and it yields $\|(q, v)\|_{\mathcal{Y}_{0}} \leq \Psi\left(t_{0}, R_{0}, \eta_{0}\right) \leq \eta_{0}$, hence $(q, v) \in M$.

In the case of the map $\mathcal{T}^{1}:\left(r^{*}, w^{*}\right) \mapsto(r, w)$ defined via solution to (59), (61),(62), (63), (64), we look for a fixed-point in the space ${ }_{0} \mathcal{Y}_{T}$. The solution can only be defined globally on $[0, T]$ if the solution to (59) remains inside of $] \varrho_{\min }, \varrho_{\max }[$ on the entire time-interval. We will show that this can be ensured if the starting perturbation $w^{*}$ satisfies an inequality of type

$$
\phi_{0}\left(T,\left\|w^{*}\right\|_{W_{p}^{2,1}\left(Q_{T}\right)}\right)\left\|w^{*}\right\|_{W_{p}^{2,1}\left(Q_{T}\right)} \leq a_{0},
$$

in which $a_{0}>0$ is a fixed number depending on the distance of the initial data to the critical values $\left\{\varrho_{\min }, \varrho_{\max }\right\}$, and $\phi_{0}$ is a continuous function on $\overline{\mathbb{R}}_{+}^{2}$, which increases in both arguments. We then prove a continuity estimate of the type

$$
\|(r, w)\|_{\mathcal{Y}_{T}} \leq \Psi\left(T, R_{0},\left\|\left(r^{*}, w^{*}\right)\right\|_{\mathcal{Y}_{T}}\right) R_{1} .
$$


Here $R_{0}$ stands for the magnitude of initial data $\left(q^{0}, \varrho_{0}\right.$ and $\left.v^{0}\right)$ and external forces $b$. The parameter $R_{1}$ expresses the distance of the initial data to a stationary/equilibrium solution (def. in (16), (17)). Defining $\eta_{0}$ to be the smallest positive solution to the equation $\phi_{0}\left(T, \eta_{0}\right) \eta_{0}=a_{0}$, we will show that $\mathcal{T}^{1}$ maps the ball of radius $\eta_{0}$ in ${ }_{0} \mathcal{Y}_{T}$ for initial data satisfying $R_{1} \leq \eta_{0} / \Psi\left(T, R_{0}, \eta_{0}\right)$. In order to apply the contraction principle and prove the theorems, we shall therefore prove the continuity estimate (65), (66). This is the main object of the next sections.

\section{Estimates of the linearised problems}

In this section, we present the estimates on which our main results in Theorem 1, 8 are footing. The preliminary work done in the paper [5] shall, in many points, allow to abridge the calculations. The main novelty is the inversion of the parabolic-elliptic subsystem, which shall be dealt with in all details.

To achieve more simplicity in the notation, we introduce both for a function or vector field $f \in W_{p}^{2,1}\left(Q_{T} ; \mathbb{R}^{k}\right)(k \in \mathbb{N})$ and $t \leq T$ the notation

$$
\mathscr{V}(t ; f):=\|f\|_{W_{p}^{2,1}\left(Q_{t} ; \mathbb{R}^{k}\right)}+\sup _{\tau \leq t}\|f(\cdot, \tau)\|_{W_{p}^{2-\frac{2}{p}}\left(\Omega ; \mathbb{R}^{k}\right)} .
$$

Recall that $W_{p}^{2-2 / p}(\Omega)$ is the trace space for $f \in W_{p}^{2,1}\left(Q_{T}\right), f \mapsto f(\cdot, t)$. Moreover we will need Hölder half-norms. For $\alpha, \beta \in[0,1]$ and $f$ scalar valued, we denote

$$
\begin{array}{r}
{[f]_{C^{\alpha}(\Omega)}:=\sup _{x \neq y \in \Omega} \frac{|f(x)-f(y)|}{|x-y|^{\alpha}}, \quad[f]_{C^{\alpha}(0, T)}:=\sup _{t \neq s \in[0, T]} \frac{|f(t)-f(s)|}{|t-s|^{\alpha}}} \\
{[f]_{C^{\alpha, \beta}\left(Q_{T}\right)}:=\sup _{t \in[0, T]}[f(\cdot, t)]_{C^{\alpha}(\Omega)}+\sup _{x \in \Omega}[f(x, \cdot)]_{C^{\beta}(0, T)} .}
\end{array}
$$

The corresponding Hölder norms $\|f\|_{C^{\alpha}(\Omega)},\|f\|_{C^{\alpha}(0, T)}$ and $f \in C^{\alpha, \beta}\left(Q_{T}\right)$ are defined by adding the corresponding $L^{\infty}$-norm to the half-norm.

\subsection{Estimates of a linearised problem for the variables $q$ and $\zeta$}

We first formulate some global assumptions and notations. Recall that $I=] \varrho_{\min }$, $\varrho_{\max }\left[\right.$. In this section, the maps $R_{q}, \tilde{M}: I \times \mathbb{R}^{N-2} \rightarrow \mathbb{R}^{(N-2) \times(N-2)}$ are assumed to be of class $C^{1}\left(I \times \mathbb{R}^{N-2}\right)$ into the set of symmetric, positive definite matrices. Furtheron, $A: I \times \mathbb{R}^{N-2} \rightarrow \mathbb{R}^{N-2}$, and $d: I \times \mathbb{R}^{N-2} \rightarrow \mathbb{R}_{+}$are of class $C^{1}$ too. We fix $p>3$, and we consider given $q^{*} \in W_{p}^{2,1}\left(Q_{T} ; \mathbb{R}^{N-2}\right)$ and $\varrho^{*} \in W_{p, \infty}^{1,1}\left(Q_{T}\right)$ such that $\left.\varrho^{*}(x, t) \in\right] \varrho_{\min }, \varrho_{\max }\left[\right.$ for all $(x, t) \in \overline{Q_{T}}$. We then denote $R_{q}^{*}:=R_{q}\left(\varrho^{*}, q^{*}\right)$, $\tilde{M}^{*}:=\tilde{M}\left(\varrho^{*}, q^{*}\right), A^{*}:=A\left(\varrho^{*}, q^{*}\right)$ and $d^{*}:=d\left(\varrho^{*}, q^{*}\right)$. For $t \leq T$, we introduce 
the positive functions

$$
\begin{aligned}
m^{*}(t) & :=m\left(\varrho^{*}, t\right):=\inf _{(x, \tau) \in Q_{t}} \min \left\{\frac{\varrho^{*}(x, \tau)}{\varrho_{\min }}-1,1-\frac{\varrho^{*}(x, \tau)}{\varrho_{\max }}\right\} \\
M^{*}(t) & :=M\left(\varrho^{*}, t\right):=\max \left\{\frac{1}{\inf _{(x, \tau) \in Q_{t}}\left(\frac{\varrho^{*}(x, \tau)}{\varrho_{\min }}-1\right)}, \frac{1}{\inf _{(x, \tau) \in Q_{t}}\left(1-\frac{\varrho^{*}(x, \tau)}{\varrho_{\max }}\right)}\right\} .
\end{aligned}
$$

We let $g \in L^{p}\left(Q_{T} ; \mathbb{R}^{N-2}\right), q^{0} \in W_{p}^{2-2 / p}\left(\Omega ; \mathbb{R}^{N-2}\right)$ such that $v \cdot \nabla q^{0}(x)=0$ on $\partial \Omega$ in the sense of traces, and $h \in W_{p}^{1,0}\left(Q_{T} ; \mathbb{R}^{3}\right)$.

For a pair $(q, \zeta): Q_{T} \rightarrow \mathbb{R}^{N-2} \times \mathbb{R}$ we consider the linear parabolic-elliptic auxiliary problem

$$
\begin{aligned}
& R_{q}^{*} \partial_{t} q-\operatorname{div}\left(\tilde{M}^{*} \nabla q+A^{*} \nabla \zeta\right)= \text { in } Q_{T}, v \cdot \nabla q=0 \text { on } S_{T}, \\
& q(x, 0)=q^{0}(x) \text { in } \Omega, \\
&-\operatorname{div}\left(d^{*} \nabla \zeta+A^{*} \nabla q\right)=-\operatorname{div} h \text { in } Q_{T}, v \cdot \nabla \zeta=0 \text { on } S_{T},
\end{aligned}
$$

and we want to obtain an estimate in the norm of $W_{p}^{2,1}\left(Q_{T} ; \mathbb{R}^{N-2}\right) \times W_{p}^{2,0}\left(Q_{T}\right)$ for the solution. To this aim we first show that (70), (71) can be equivalently reformulated as a system coupled only in the lower order.

Lemma 10. We adopt the general assumptions and notations formulated at the beginning of this section. A pair $(q, \zeta) \in W_{p}^{2,1}\left(Q_{T} ; \mathbb{R}^{N-2}\right) \times W_{p}^{2,0}\left(Q_{T}\right)$ is a solution to the problem (70), (71) if the identity (71) and the initial and boundary condition are satisfied, and if instead of (70) we have

$$
\begin{aligned}
& R_{q}^{*} \partial_{t} q-\operatorname{div}\left(\left[\tilde{M}^{*}-\frac{A^{*} \otimes A^{*}}{d^{*}}\right] \nabla q\right) \\
& \quad=g+\nabla \zeta \cdot\left[\nabla A^{*}-\frac{A^{*}}{d^{*}} \nabla d^{*}\right]+\nabla\left(\frac{A^{*}}{d^{*}}\right) \cdot \nabla q A^{*}+\frac{A^{*}}{d^{*}} \operatorname{div} h .
\end{aligned}
$$

Proof. Computing the derivatives in the elliptic equation (71), we obtain that

$$
-d^{*} \triangle \zeta=\nabla d^{*} \cdot \nabla \zeta+\operatorname{div}\left(A^{*} \nabla q\right)-\operatorname{div} h .
$$

Thus, under the side-condition (73), the parabolic equations (70) are equivalent to

$$
\begin{aligned}
& R_{q}^{*} \partial_{t} q-\operatorname{div}\left(\tilde{M}^{*} \nabla q\right)=g+A^{*} \Delta \zeta+\nabla A^{*} \cdot \nabla \zeta \\
& \quad=g+\nabla A^{*} \cdot \nabla \zeta-\frac{1}{d^{*}} A^{*}\left[\nabla d^{*} \cdot \nabla \zeta+\operatorname{div}\left(A^{*} \nabla q\right)-\operatorname{div} h\right]
\end{aligned}
$$

Use of $\frac{A^{*}}{d^{*}} \operatorname{div}\left(A^{*} \nabla q\right)=\operatorname{div}\left(\frac{A^{*} \otimes A^{*}}{d^{*}} \nabla q\right)-A^{*} \nabla q \cdot \nabla\left(\frac{A^{*}}{d^{*}}\right)$ yields the claim.

Using this lemma, we next prove an estimate for the solution to the linearised parabolic-elliptic problem. 
Proposition 11. Under the general assumptions and notations of this section, there is a unique pair $(q, \zeta) \in W_{p}^{2,1}\left(Q_{T} ; \mathbb{R}^{N-2}\right) \times W_{p}^{2,0}\left(Q_{T}\right)$, solution to the problem (70), (71), such that $\int_{\Omega} \zeta(x, t) d x=0$ for all $\left.t \in\right] 0, T[$. Moreover, there are a constant $C$ depending only on $\Omega$, and continuous functions $\Psi_{1}=\Psi_{1}\left(t, a_{1}, \ldots, a_{5}\right)$ and $\Phi=\Phi\left(t, a_{1}, \ldots, a_{5}\right)$ defined for all $t \geq 0$ and all numbers $a_{1}, \ldots, a_{5} \geq 0$, such that for all $t \leq T$ and for $0<\beta \leq 1$ arbitrary:

$$
\begin{gathered}
\mathscr{V}(t ; q)+\|\zeta\|_{W_{p}^{2,0}\left(Q_{t}\right)} \leq C \Psi_{1, t}\left(1+\left[\varrho^{*}\right]_{C^{\beta, \frac{\beta}{2}}\left(Q_{t}\right)}\right)^{\frac{2}{\beta}}\left(\left\|q^{0}\right\|_{W_{p}^{2-\frac{2}{p}}(\Omega)}+\|g\|_{L^{p}\left(Q_{t}\right)}\right. \\
\left.+\|h\|_{W_{p}^{1,0}\left(Q_{t}\right)}\right)+C \Phi_{t}\|h\|_{L^{p}\left(Q_{t}\right)}, \\
\Psi_{1, t}=\Psi_{1}\left(t, M^{*}(t),\left\|q^{*}(0)\right\|_{C^{\beta}(\Omega)}, \mathscr{V}\left(t ; q^{*}\right),\left[\varrho^{*}\right]_{C^{\beta, \frac{\beta}{2}}\left(Q_{t}\right)},\left\|\nabla \varrho^{*}\right\|_{L^{p, \infty}\left(Q_{t}\right)}\right), \\
\Phi_{t}=\Phi\left(t, M^{*}(t),\left\|q^{*}(0)\right\|_{C^{\beta}(\Omega)}, \mathscr{V}\left(t ; q^{*}\right),\left[\varrho^{*}\right]_{C^{\beta, \frac{\beta}{2}}\left(Q_{t}\right)},\left\|\nabla \varrho^{*}\right\|_{L^{p, \infty}\left(Q_{t}\right)}\right) .
\end{gathered}
$$

The function $\Psi_{1}$ possesses moreover the following two properties: It is increasing in all arguments, and the value of $\Psi_{1}\left(0, a_{1}, \ldots, a_{5}\right)=\Psi_{1}^{0}\left(a_{1}, a_{2}\right)$ is a function independent on the three last arguments. The function $\Phi$ is increasing in all arguments.

Proof. The existence and uniqueness can be easily obtained by means of the uniform estimates. We thus suppose first that $(q, \zeta) \in W_{p}^{2,1}\left(Q_{T} ; \mathbb{R}^{N-2}\right) \times W_{p}^{2,0}\left(Q_{T}\right)$ is a given solution, and we prove the claimed estimate. In order to simplify the discussion, we adopt the following convention: When computing the derivative of a coefficient, like $\nabla d^{*}=d_{\varrho}^{*} \nabla \varrho^{*}+d_{q}^{*} \nabla q^{*}$, there occur different functions $d_{\varrho}^{*}:=d_{\varrho}\left(\varrho^{*}, q^{*}\right)$ or $d_{q_{j}}^{*}=d_{q_{j}}\left(\varrho^{*}, q^{*}\right)$ of the variables $\varrho^{*}, q^{*}$. We denote $c_{1}^{*}=c_{1}\left(M^{*}(t),\left\|q^{*}\right\|_{L^{\infty}\left(Q_{t}\right)}\right)$ a generic continuous function depending only on $M^{*}(t)$ and $\left\|q^{*}\right\|_{L^{\infty}\left(Q_{t}\right)}$, and increasing in these arguments. We then bound the $L^{\infty}\left(Q_{T}\right)$ norms of all nonlinear functions depending on $\varrho^{*}, q^{*}$ by this generic $c_{1}^{*}$.

Step 1: First estimate for the variable $\zeta$.

For almost all $s \leq t$, the function $\zeta$ satisfies the weak Neumann problem

$$
\int_{\Omega} d^{*} \nabla \zeta(x, s) \cdot \nabla \phi(x) d x=\int_{\Omega}\left(-A^{*} \nabla q+h\right)(x, s) \cdot \nabla \phi(x) d x .
$$

By well-known weak elliptic theory, there is a unique solution $\zeta(s) \in W^{1, p}(\Omega)$ with $\int_{\Omega} \zeta(x, s) d x=0$. Moreover, for all $0<\beta<1$, perturbation techniques shortly recalled in Appendix, Lemma 28 yield the estimate

$$
\begin{aligned}
& \|\nabla \zeta(s)\|_{L^{p}(\Omega)} \leq c\left(\Omega, p, \inf _{\Omega} d^{*}(s), \sup _{\Omega} d^{*}(s)\right)\left(1+\left[d^{*}(s)\right]_{C^{\beta}(\Omega)}\right)^{\frac{1}{\beta}} \times \\
& \quad \times\left(\left\|A^{*} \nabla q(s)\right\|_{L^{p}(\Omega)}+\|h(s)\|_{L^{p}(\Omega)}\right) \\
& \leq c_{1}^{*}\left(1+\left[d^{*}(s)\right]_{C^{\beta}(\Omega)}\right)^{\frac{1}{\beta}}\left(\left\|A^{*}(s)\right\|_{L^{\infty}(\Omega)}\|\nabla q(s)\|_{L^{p}(\Omega)}+\|h(s)\|_{L^{p}(\Omega)}\right) .
\end{aligned}
$$

We define $\phi_{t}^{*}:=\sup _{s \leq t}\left(1+\left[d^{*}(s)\right]_{C^{\beta}(\Omega)}\right)^{\frac{1}{\beta}}$. We bound $\sup _{s \leq t}\left\|A^{*}(s)\right\|_{L^{\infty}(\Omega)}$ with a generic $c_{1}^{*}$, and it follows that

$$
\|\zeta\|_{W_{p}^{1,0}\left(Q_{t}\right)} \leq c_{1}^{*} \phi_{t}^{*}\left(\|\nabla q\|_{L^{p}\left(Q_{t}\right)}+\|h\|_{L^{p}\left(Q_{t}\right)}\right)
$$


Step 2: First bound for the variable $q$.

We start from $(72)$, and we define $K(\varrho, q):=\tilde{M}(\varrho, q)-A(\varrho, q) \otimes A(\varrho, q) / d(\varrho, q)$. In view of the definitions (22), (23), (24), $K \in \mathbb{R}^{(N-2) \times(N-2)}$ is obviously symmetric, and obeys

$$
K=\Pi^{\top} M \Pi-\frac{\Pi^{\top} M \bar{V} \otimes \Pi^{\top} M \bar{V}}{M \bar{V} \cdot \bar{V}} .
$$

For all $y \in \mathbb{R}^{N-2}, K y \cdot y=M \Pi y \cdot \Pi y-(M \bar{V} \cdot \Pi y)^{2} / M \bar{V} \cdot \bar{V} \geq 0$, because $M$ is positive semi-definite. By the Cauchy-Schwarz inequality, $K y \cdot y=0$ is possible only if either $\Pi y$ and $\bar{V}$ are parallel, or if $\Pi y$ and $1^{N}$ are parallel. Recall in this place that $\Pi y=\sum_{k=1}^{N-2} y_{k} \xi^{k}$. By the choice of the $\xi^{k}$ s, we know that $\left\{\xi^{1}, \ldots, \xi^{N-2}, \bar{V}, 1^{N}\right\}$ is a basis of $\mathbb{R}^{N}$. Thus, $\Pi y=\lambda \bar{V}$ or $\Pi y=\lambda 1^{N}$ both would imply that $y=0$. This shows that $K y \cdot y>0$ unless $y=0$, hence $K$ is positive definite.

Defining $K^{*}:=K\left(\varrho^{*}, q^{*}\right)$, we rephrase (72) as

$$
R_{q}^{*} \partial_{t} q-\operatorname{div}\left(K^{*} \nabla q\right)=g+\tilde{g}
$$

in which $\tilde{g}:=\nabla \zeta \cdot\left[\nabla A^{*}-\frac{A^{*}}{d^{*}} \nabla d^{*}\right]+\nabla\left(\frac{A^{*}}{d^{*}}\right) \cdot \nabla q A^{*}+\frac{A^{*}}{d^{*}} \operatorname{div} h$ is bounded via

$$
|\tilde{g}| \leq c_{1}^{*}\left(\left|\nabla \zeta \cdot \nabla \varrho^{*}\right|+\left|\nabla \zeta \cdot \nabla q^{*}\right|+\left|\nabla q \cdot \nabla \varrho^{*}\right|+\left|\nabla q \cdot \nabla q^{*}\right|+|\nabla h|\right) .
$$

We now apply Appendix, Lemma 26, which basically recalls the result of [5], Prop. 7.1 for a similar parabolic system. With $D_{0}(t):=\left(1+\left[\varrho^{*}\right]_{C^{\beta, \beta / 2}\left(Q_{t}\right)}\right)^{2 / \beta}\left\|q^{0}\right\|_{W_{p}^{2-2 / p}(\Omega)}+$ $\|g\|_{L^{p}\left(Q_{t}\right)}$, and using (77) to bound the norm of $\tilde{g}$, we obtain for the solution to (76)

$$
\begin{aligned}
\mathscr{V}(t ; q) \leq & C \bar{\Psi}_{1, t}\left[D_{0}(t)+c_{1}^{*}\|\nabla h\|_{L^{p}\left(Q_{t}\right)}+c_{1}^{*}\left(\left\|\nabla \zeta \cdot \nabla \varrho^{*}\right\|_{L^{p}\left(Q_{t}\right)}+\left\|\nabla \zeta \cdot \nabla q^{*}\right\|_{L^{p}\left(Q_{t}\right)}\right.\right. \\
& \left.\left.+\left\|\nabla q \cdot \nabla \varrho^{*}\right\|_{L^{p}\left(Q_{t}\right)}+\left\|\nabla q \cdot \nabla q^{*}\right\|_{L^{p}\left(Q_{t}\right)}\right)\right]
\end{aligned}
$$

where $\bar{\Psi}_{1, t}=\bar{\Psi}_{1}\left(t, M^{*}(t),\left\|q^{*}(0)\right\|_{C^{\beta}(\Omega)}, \mathscr{V}\left(t ; q^{*}\right),\left[\varrho^{*}\right]_{C^{\beta, \beta / 2}\left(Q_{t}\right)},\left\|\nabla \varrho^{*}\right\|_{L^{p, \infty}\left(Q_{t}\right)}\right)$, and the function $\bar{\Psi}_{1}$ fulfills all structural assumptions stated for $\Psi_{1}$.

Step 3: Main estimate for the variable $\zeta$.

Since $\zeta \in W_{p}^{2,0}\left(Q_{T}\right)$, we can employ the pointwise identity (73). Since $\zeta$ has mean-value zero for all times, the full $W^{2, p}$ norm can be estimated by the NeumannLaplacian, and we obtain that

$$
\begin{aligned}
\|\zeta\|_{W_{p}^{2,0}\left(Q_{t}\right)} & \leq c(\Omega, p)\|-\Delta \zeta\|_{L^{p}\left(Q_{t}\right)} \\
& =c(\Omega, p)\left\|\left(d^{*}\right)^{-1}\left(\nabla d^{*} \cdot \nabla \zeta+\operatorname{div}\left(A^{*} \nabla q-h\right)\right)\right\|_{L^{p}\left(Q_{t}\right)} \\
& \leq c \frac{1}{\inf _{(x, s) \in Q_{t}} d^{*}(x, s)}\left(\left\|\nabla d^{*} \cdot \nabla \zeta\right\|_{L^{p}\left(Q_{t}\right)}+\left\|\operatorname{div}\left(A^{*} \nabla q-h\right)\right\|_{L^{p}\left(Q_{t}\right)}\right) .
\end{aligned}
$$


Computing the derivatives of the coefficients, and using the same conventions as above, we derive from (79) the inequality

$$
\begin{aligned}
& \|\zeta\|_{W_{p}^{2,0}\left(Q_{t}\right)} \leq c_{1}^{*}\left(\|\Delta q\|_{L^{p}\left(Q_{t}\right)}+\|\operatorname{div} h\|_{L^{p}\left(Q_{t}\right)}\right) \\
& \quad+c_{1}^{*}\left(\left\|\nabla \varrho^{*} \cdot \nabla \zeta\right\|_{L^{p}\left(Q_{t}\right)}+\left\|\nabla q^{*} \cdot \nabla \zeta\right\|_{L^{p}\left(Q_{t}\right)}+\left\|\nabla \varrho^{*} \cdot \nabla q\right\|_{L^{p}\left(Q_{t}\right)}\right. \\
& \left.\quad+\left\|\nabla q^{*} \cdot \nabla q\right\|_{L^{p}\left(Q_{t}\right)}\right)
\end{aligned}
$$

We estimate $\|\triangle q\|_{L^{p}\left(Q_{t}\right)} \leq \mathscr{V}(t ; q)$, then we employ the inequality (78) to see that

$$
\begin{aligned}
\|\zeta\|_{W_{p}^{2,0}\left(Q_{t}\right) \leq} & C \bar{\Psi}_{1, t} D_{0}(t)+c_{1}^{*}\left(1+C \bar{\Psi}_{1, t}\right)\|\nabla h\|_{L^{p}\left(Q_{t}\right)} \\
& +c_{1}^{*}\left(1+C \bar{\Psi}_{1, t}\right)\left(\left\|\nabla \varrho^{*} \cdot \nabla \zeta\right\|_{L^{p}\left(Q_{t}\right)}+\left\|\nabla q^{*} \cdot \nabla \zeta\right\|_{L^{p}\left(Q_{t}\right)}\right) \\
& +c_{1}^{*}\left(1+C \bar{\Psi}_{1, t}\right)\left(\left\|\nabla \varrho^{*} \cdot \nabla q\right\|_{L^{p}\left(Q_{t}\right)}+\left\|\nabla q^{*} \cdot \nabla q\right\|_{L^{p}\left(Q_{t}\right)}\right)
\end{aligned}
$$

Step 4: Combined estimates.

We add (78) to (80) to obtain that

$$
\begin{aligned}
\mathscr{V}(t ; q)+\|\zeta\|_{W_{p}^{2,0}\left(Q_{t}\right) \leq} & 2 C \bar{\Psi}_{1, t} D_{0}(t)+c_{1}^{*}\left(1+2 C \bar{\Psi}_{1, t}\right)\|\nabla h\|_{L^{p}\left(Q_{t}\right)} \\
& +c_{1}^{*}\left(1+2 C \bar{\Psi}_{1, t}\right)\left(\left\|\nabla \varrho^{*} \cdot \nabla \zeta\right\|_{L^{p}\left(Q_{t}\right)}+\left\|\nabla q^{*} \cdot \nabla \zeta\right\|_{L^{p}\left(Q_{t}\right)}\right) \\
& +c_{1}^{*}\left(1+2 C \bar{\Psi}_{1, t}\right)\left(\left\|\nabla \varrho^{*} \cdot \nabla q\right\|_{L^{p}\left(Q_{t}\right)}+\left\|\nabla q^{*} \cdot \nabla q\right\|_{L^{p}\left(Q_{t}\right)}\right)
\end{aligned}
$$

In order to control the factors on the right-hand, we first apply (33) to find that $\|\nabla \zeta(s)\|_{L^{\infty}(\Omega)} \leq C_{1}\left\|D^{2} \zeta(s)\right\|_{L^{p}(\Omega)}^{\alpha}\|\zeta(s)\|_{L^{p}(\Omega)}^{1-\alpha}+C_{2}\|\zeta(s)\|_{L^{p}(\Omega)}, \quad \alpha:=\frac{1}{2}+\frac{3}{2 p}$, with $C_{i}=C_{i}(\Omega), i=1,2$. We can bound $a b \leq \epsilon a^{1 / \alpha}+c_{\alpha} \epsilon^{-\alpha /(1-\alpha)} b^{1 /(1-\alpha)}$ (Young's inequality), for all $\epsilon>0$ and $a, b>0$. By these means, it follows that

$$
\begin{aligned}
& \left\|\nabla \varrho^{*} \cdot \nabla \zeta\right\|_{L^{p}\left(Q_{t}\right)}^{p} \leq \int_{0}^{t}\left|\nabla \varrho^{*}(s)\right|_{p}^{p}|\nabla \zeta(s)|_{\infty}^{p} d s \\
& \quad \leq C_{1} \int_{0}^{t}\left|\nabla \varrho^{*}(s)\right|_{p}^{p}\left|D^{2} \zeta(s)\right|_{p}^{p \alpha}|\zeta(s)|_{p}^{p(1-\alpha)} d s+C_{2} \int_{0}^{t}\left|\nabla \varrho^{*}(s)\right|_{p}^{p}|\zeta(s)|_{p}^{p} d s \\
& \quad \leq \epsilon \int_{0}^{t}\left|D^{2} \zeta(s)\right|_{p}^{p} d s+\left.c_{\alpha} \epsilon^{-\frac{\alpha}{1-\alpha}} \int_{0}^{t}\left|\nabla \varrho^{*}(s)\right|\right|_{p} ^{\frac{p}{1-\alpha}}|\zeta(s)|_{p}^{p} d s+C_{2} \int_{0}^{t}\left|\nabla \varrho^{*}(s)\right|_{p}^{p}|\zeta(s)|_{p}^{p} d s \\
& \quad \leq \epsilon \int_{0}^{t}\left|D^{2} \zeta(s)\right|_{p}^{p} d s+\int_{0}^{t}|\zeta(s)|_{p}^{p}\left(c_{\alpha} \epsilon^{-\frac{\alpha}{1-\alpha}}\left|\nabla \varrho^{*}(s)\right|_{p}^{\frac{p}{1-\alpha}}+C_{2}\left|\nabla \varrho^{*}(s)\right|_{p}^{p}\right) d s .
\end{aligned}
$$

Here we use the abbreviation $|\cdot|_{r}$ for $\|\cdot\|_{L^{r}(\Omega)}$. Just in the same way, we show that

$$
\begin{aligned}
& \left\|\nabla q^{*} \cdot \nabla \zeta\right\|_{L^{p}\left(Q_{t}\right)}^{p} \\
& \quad \leq \epsilon \int_{0}^{t}\left|D^{2} \zeta(s)\right|_{p}^{p} d s+\int_{0}^{t}|\zeta(s)|_{p}^{p}\left(c_{\alpha} \epsilon^{-\frac{\alpha}{1-\alpha}}\left|\nabla q^{*}(s)\right|_{p}^{\frac{p}{1-\alpha}}+C_{2}\left|\nabla q^{*}(s)\right|_{p}^{p}\right) d s .
\end{aligned}
$$


We let

$F^{*}(t):=\sup _{s \leq t}\left(\left|\nabla q^{*}(s)\right|_{L^{p}}^{p}+\left|\nabla \varrho^{*}(s)\right|_{L^{p}}^{p}\right), \quad X^{*}(t ; \zeta):=\left\|\nabla \varrho^{*} \cdot \nabla \zeta\right\|_{L^{p}\left(Q_{t}\right)}^{p}+\left\|\nabla q^{*} \cdot \nabla \zeta\right\|_{L^{p}\left(Q_{t}\right)}^{p}$.

With the help of (82) and of (83), it follows that

$$
X^{*}(t ; \zeta) \leq 2 \epsilon\left\|D^{2} \zeta(s)\right\|_{L^{p}\left(Q_{t}\right)}^{p}+\left[c_{\alpha} \epsilon^{-\frac{\alpha}{1-\alpha}}\left(F^{*}(t)\right)^{\frac{1}{1-\alpha}}+C_{2} F^{*}(t)\right]\|\zeta\|_{L^{p}\left(Q_{t}\right)}^{p} .
$$

We choose $\epsilon=2^{-2-1 / p}\left(c_{1}^{*}\left(1+2 C \bar{\Psi}_{1, t}\right)\right)^{-p}$, where $c_{1}^{*}, C$ and $\bar{\Psi}_{1, t}$ are the numbers occurring in the relation (81). Then

$$
\begin{aligned}
& \left(c_{1}^{*}\left(1+2 C \bar{\Psi}_{1, t}\right)\right)^{p} X^{*}(t ; \zeta) \leq \frac{1}{2^{1+1 / p}}\|\zeta\|_{W_{p}^{2,0}\left(Q_{t}\right)}^{p} \\
& \quad+\left(c_{1}^{*}\left(1+2 C \bar{\Psi}_{1, t}\right)\right)^{p}\left[c_{\alpha}\left(2^{2+1 / p}\left(c_{1}^{*}\left(1+2 C \bar{\Psi}_{1, t}\right)\right)^{p}\right)^{\frac{\alpha}{1-\alpha}}\left(F^{*}(t)\right)^{\frac{1}{1-\alpha}}+C_{2} F^{*}(t)\right]\|\zeta\|_{L^{p}}^{p}\left(Q_{t}\right)
\end{aligned}
$$

Due to our conventions, we can bound every power of $c_{1}^{*}$ and the maximum of 1 and $c_{1}^{*}$ again by another such function. Introducing a factor

$$
\begin{aligned}
\left(\Phi_{1, t}^{*}\right)^{p}:= & c_{1}^{*}\left(1+2 C \bar{\Psi}_{1, t}\right)^{p} \max \left\{c_{\alpha}\left(2^{2+1 / p}\left(1+2 C \bar{\Psi}_{1, t}\right)^{p}\right)^{\frac{\alpha}{1-\alpha}}, C_{2}\right\} \\
& \times\left\{\left(\mathscr{V}^{p}\left(t ; q^{*}\right)+\left\|\nabla \varrho^{*}\right\|_{L^{p, \infty}\left(Q_{t}\right)}^{p}\right)^{\frac{1}{1-\alpha}}+\left(\mathscr{V}^{p}\left(t ; q^{*}\right)+\left\|\nabla \varrho^{*}\right\|_{L^{p, \infty}\left(Q_{t}\right)}^{p}\right),\right.
\end{aligned}
$$

we can rephrase (84) as

$$
\left(c_{1}^{*}\left(1+2 C \bar{\Psi}_{1, t}\right)\right)^{p} X^{*}(t ; \zeta) \leq \frac{1}{2^{1+1 / p}}\|\zeta\|_{W_{p}^{2,0}\left(Q_{t}\right)}^{p}+\left(\Phi_{1, t}^{*}\right)^{p}\|\zeta\|_{L^{p}\left(Q_{t}\right)}^{p} .
$$

By means of (75), we bound $\|\zeta\|_{L^{p}\left(Q_{t}\right)} \leq c_{1}^{*} \phi_{t}^{*}\left(\|\nabla q\|_{L^{p}\left(Q_{t}\right)}+\|h\|_{L^{p}\left(Q_{t}\right)}\right)$. Raising (85) to the power $1 / p$, we show that

$$
\begin{aligned}
c_{1}^{*}(1 & \left.+2 C \bar{\Psi}_{1, t}\right)\left(\left\|\nabla \varrho^{*} \cdot \nabla \zeta\right\|_{L^{p}\left(Q_{t}\right)}+\left\|\nabla q^{*} \cdot \nabla \zeta\right\|_{L^{p}\left(Q_{t}\right)}\right) \\
& \leq \frac{1}{2}\|\zeta\|_{W_{p}^{2,0}\left(Q_{t}\right)}+C_{p} c_{1}^{*} \phi_{t}^{*} \Phi_{1, t}^{*}\left(\|\nabla q\|_{L^{p}\left(Q_{t}\right)}+\|h\|_{L^{p}\left(Q_{t}\right)} .\right.
\end{aligned}
$$

We insert the latter result into (81), obtaining

$$
\begin{aligned}
\mathscr{V}(t ; q)+ & \frac{1}{2}\|\zeta\|_{W_{p}^{2,0}\left(Q_{t}\right)} \leq 2 C \bar{\Psi}_{1, t} D_{0}(t) \\
& +c_{1}^{*}\left(1+2 C \bar{\Psi}_{1, t}\right)\|\nabla h\|_{L^{p}\left(Q_{t}\right)}+C_{p} c_{1}^{*} \phi_{t}^{*} \Phi_{1, t}^{*}\|h\|_{L^{p}\left(Q_{t}\right)} \\
& +c_{1}^{*}\left(1+2 C \bar{\Psi}_{1, t}\right)\left(\left\|\nabla \varrho^{*} \cdot \nabla q\right\|_{L^{p}\left(Q_{t}\right)}+\left\|\nabla q^{*} \cdot \nabla q\right\|_{L^{p}\left(Q_{t}\right)}\right)+C_{p} c_{1}^{*} \phi_{t}^{*} \Phi_{1, t}^{*}\|\nabla q\|_{L^{p}\left(Q_{t}\right)} .
\end{aligned}
$$

In order to estimate $X^{*}(t, q)$, we apply the same steps as for $X^{*}(t, \zeta)$ (cf. (85)). Hence

$$
\left(c_{1}^{*}\left(1+2 C \bar{\Psi}_{1, t}\right)\right)^{p} X^{*}(t ; q) \leq \frac{1}{2^{1+1 / p}}\|q\|_{W_{p}^{2,0}\left(Q_{t}\right)}^{p}+\left(\Phi_{1, t}^{*}\right)^{p}\|q\|_{L^{p}\left(Q_{t}\right)}^{p},
$$


which, after raising to the power $1 / p$, yields $c_{1}^{*}\left(1+2 C \bar{\Psi}_{1, t}\right)\left(\left\|\nabla \varrho^{*} \cdot \nabla q\right\|_{L^{p}\left(Q_{t}\right)}+\left\|\nabla q^{*} \cdot \nabla q\right\|_{L^{p}\left(Q_{t}\right)}\right) \leq \frac{1}{2}\|q\|_{W_{p}^{2,0}\left(Q_{t}\right)}+C_{p} \Phi_{1, t}^{*}\|q\|_{L^{p}\left(Q_{t}\right)}$.

Since $\|q\|_{W_{p}^{2,0}\left(Q_{t}\right)} \leq \mathscr{V}(t ; q)$, the latter and (86) imply that

$$
\begin{aligned}
& \frac{1}{2}\left(\mathscr{V}(t ; q)+\|\zeta\|_{W_{p}^{2,0}\left(Q_{t}\right)}\right) \leq C_{p} \Phi_{1, t}^{*}\left(1+c_{1}^{*} \phi_{t}^{*}\right)\|q\|_{W_{p}^{1,0}\left(Q_{t}\right)} \\
& \quad+2 C \bar{\Psi}_{1, t} D_{0}(t)+c_{1}^{*}\left(1+2 C \bar{\Psi}_{1, t}\right)\|\nabla h\|_{L^{p}\left(Q_{t}\right)}+C_{p} c_{1}^{*} \phi_{t}^{*} \Phi_{1, t}^{*}\|h\|_{L^{p}\left(Q_{t}\right)} .
\end{aligned}
$$

In order to finally get rid of the factors with $q$ on the right-hand side, we introduce

$$
[A(t)]^{\frac{1}{p}}:=2 C \bar{\Psi}_{1, t} D_{0}(t)+c_{1}^{*}\left(1+2 C \bar{\Psi}_{1, t}\right)\|\nabla h\|_{L^{p}\left(Q_{t}\right)}+C_{p} c_{1}^{*} \phi_{t}^{*} \Phi_{1, t}^{*}\|h\|_{L^{p}\left(Q_{t}\right)},
$$

and

$$
[B(t)]^{\frac{1}{p}}:=C_{p} \Phi_{1, t}^{*}\left(1+c_{1}^{*} \phi_{t}^{*}\right), \quad f(t):=\sup _{\tau \leq t}\|q(\tau)\|_{W_{p}^{2-\frac{2}{p}}(\Omega)}^{p} .
$$

We raise (87) to the $p^{\text {th }}$ power. We use $f(t) \leq \mathscr{V}^{p}(t ; q)$ and $\|q\|_{W_{p}^{1,0}\left(Q_{t}\right)}^{p} \leq \int_{0}^{t} f(\tau) d \tau$. In this way, we obtain the inequality $f(t) \leq 2^{p} A(t)+2^{p} B(t) \int_{0}^{t} f(\tau) d \tau$. Using that $A$ and $B$ are monotone increasing by construction, the Gronwall Lemma yields $f(t) \leq 2^{p} A(t) \exp \left(2^{p} t B(t)\right)$. In particular, we conclude that

$$
\|q\|_{W_{p}^{1,0}\left(Q_{t}\right)} \leq[f(t) t]^{\frac{1}{p}} \leq c_{p} t^{\frac{1}{p}}[A(t)]^{\frac{1}{p}} \exp \left(\frac{2^{p}}{p} t B(t)\right) .
$$

Combining the latter with (87), it follows that

$$
\begin{aligned}
& \mathscr{V}(t ; q)+\|\zeta\|_{W_{p}^{2,0}\left(Q_{t}\right)} \leq 2\left\{1+\tilde{c}_{p} t^{\frac{1}{p}} \exp \left(\frac{2^{p}}{p} t B(t)\right)[B(t)]^{\frac{1}{p}}\right\} \\
& \quad \times\left\{2 C \bar{\Psi}_{1, t} D_{0}(t)+c_{1}^{*}\left(1+2 C \bar{\Psi}_{1, t}\right)\|\nabla h\|_{L^{p}\left(Q_{t}\right)}+C_{p} c_{1}^{*} \phi_{t}^{*} \Phi_{1, t}^{*}\|h\|_{L^{p}\left(Q_{t}\right)}\right\} .
\end{aligned}
$$

In order to verify that the factors occurring in the latter inequality possess the structure as claimed in the statement, we note that occurrences of $B(t)$ in (88) are multiplied by a power of $t$, so that they do not occur at $t=0$. Moreover, the factor $\bar{\Psi}_{1, t}$ possesses the structure required for $\Psi_{1, t}$ in the statement. In order to estimate the dependence of $\left\|q^{*}\right\|_{L^{\infty}\left(Q_{t}\right)}$ on the coefficients $c_{1}^{*}$, we apply the same strategy as in the section 7 of [5]: $\left\|q^{*}\right\|_{L^{\infty}\left(Q_{t}\right)} \leq\left\|q^{0}\right\|_{L^{\infty}(\Omega)}+t^{\gamma} \mathscr{V}\left(t ; q^{*}\right)$ (Lemma 27). Setting $\Phi_{t}:=C_{p} c_{1}^{*} \phi_{t}^{*} \Phi_{1, t}^{*}$, we are done.

\subsection{Estimates for linearised problems for the variables $v$ and $\varrho$}

First we state the estimate for the linearised momentum equation. The proof follows the lines of the corresponding result in [5]. (Since we can assume $\varrho^{*} \in\left[\varrho_{\min }, \varrho_{\max }\right]$, the proof is actually simpler.) 
Proposition 12. Assume that $\varrho^{*} \in C^{\alpha, 0}\left(Q_{t}\right)(0<\alpha \leq 1)$ attains values in $] \varrho_{\text {min }}$, $\varrho_{\max }\left[\right.$, that $f \in L^{p}\left(Q_{T} ; \mathbb{R}^{3}\right)$, and that $v^{0} \in W_{p}^{2-2 / p}\left(\Omega ; \mathbb{R}^{3}\right)$ is such that $v^{0}=0$ on $\partial \Omega$. Then, there is a unique solution $v \in W_{p}^{2,1}\left(Q_{T} ; \mathbb{R}^{3}\right)$ to $\varrho^{*} \partial_{t} v-\operatorname{div} \mathbb{S}(\nabla v)=f$ in $Q_{T}$ with the boundary conditions $v=0$ on $S_{T}$ and $v(x, 0)=v^{0}(x)$ in $\Omega$. Moreover, there is $C$ independent on $t, \varrho^{*}, v^{0}, f$ and $v$ such that

$$
\mathscr{V}(t ; v) \leq C \Psi_{2}\left(t, \sup _{\tau \leq t}\left[\varrho^{*}(\tau)\right]_{C^{\alpha}(\Omega)}\right)\left(1+\sup _{\tau \leq t}\left[\varrho^{*}(\tau)\right]_{C^{\alpha}(\Omega)}\right)^{\frac{2}{\alpha}}\left(\|f\|_{L^{p}\left(Q_{t}\right)}+\left\|v^{0}\right\|_{W_{p}^{2-2 / p}(\Omega)}\right) .
$$

The function $\Psi_{2}$ is continuous and increasing in both arguments, and it can be chosen such that $\Psi_{2}(0, a)=\left(\min \left\{1, \varrho_{\min }\right\}\right)^{-\frac{2}{\alpha}}\left(\varrho_{\max } / \varrho_{\min }\right)^{\frac{p+1}{p}}$ is independent of a.

For the linearised continuity equation, we must acknowledge the main difference with respect to the analysis of the compressible models.

Proposition 13. Assume that $v^{*} \in W_{p}^{2,1}\left(Q_{T} ; \mathbb{R}^{3}\right)$ and that $\varrho_{0} \in W^{1, p}(\Omega)$ satisfies $\varrho_{\min }<\varrho_{0}(x)<\varrho_{\max }$ in $\bar{\Omega}$. We define $M_{0}=M\left(\varrho_{0}, 0\right):=\left[\inf _{x \in \Omega}\left\{\varrho_{0}(x) / \varrho_{\min }-\right.\right.$ $\left.\left.1,1-\varrho_{0}(x) / \varrho_{\max }\right\}\right]^{-1}$. Then the problem $\partial_{t} \varrho+\operatorname{div}\left(\varrho v^{*}\right)=0$ in $Q_{T}$ with $\varrho(x, 0)=$ $\varrho_{0}(x)$ in $\Omega$ possesses a unique strictly positive solution of class $W_{p, \infty}^{1,1}\left(Q_{T}\right)$. Define also $M(t):=M(\varrho, t)(c f$. (69)). Then, we can find a constant $c$ depending only on $\Omega$ and a function $\Psi_{3}=\Psi_{3}\left(t, a_{1}, a_{2}\right)$ continuous and finite in the set

$$
\left\{t, a_{1}, a_{2} \geq 0: c a_{1} t^{1-\frac{1}{p}} a_{2} e^{c t^{1-\frac{1}{p}} a_{2}}<1\right\},
$$

such that $M(t) \leq \Psi_{3}\left(t, M_{0}, \mathscr{V}\left(t ; v^{*}\right)\right)$. Moreover, for $\beta=1-3 / p$, there are $\Psi_{4}, \Psi_{5}$ depending on $t,\left\|\nabla \varrho_{0}\right\|_{L^{p}(\Omega)}$ and $\mathscr{V}\left(t ; v^{*}\right)$ such that

$\|\nabla \varrho\|_{L^{p, \infty}\left(Q_{t}\right)} \leq \Psi_{4}\left(t,\left\|\nabla \varrho_{0}\right\|_{L^{p}(\Omega)}, \mathscr{V}\left(t ; v^{*}\right)\right), \quad[\varrho]_{C^{\beta, \frac{\beta}{2}}\left(Q_{t}\right)} \leq \Psi_{5}\left(t,\left\|\nabla \varrho_{0}\right\|_{L^{p}(\Omega)}, \mathscr{V}\left(t ; v^{*}\right)\right)$.

For $i=3,4,5, \Psi_{i}$ is continuous and increasing in all variables, and $\Psi_{i}\left(0, a_{1}, a_{2}\right)=$ $\Psi_{i}^{0}\left(a_{1}\right)$ is independent on the last variable. The identity $\Psi_{4}\left(0, a_{1}, a_{2}\right)=a_{1}$, and the inequality $\Psi_{5}\left(0, a_{1}, a_{2}\right) \leq C a_{1}$, are also valid.

Proof. The existence statement as well as the construction of the functions $\Psi_{4}$ and $\Psi_{5}$ is proved in [5], Corollary 7.8. The critical point is the construction of the function $\Psi_{3}$. We start from the well-known representation of the solution to the continuity equation (see a. o. [29])

$$
\varrho(x, t):=\varrho_{0}(y(0 ; x, t)) \exp \left(-\int_{0}^{t} \operatorname{div} v^{*}(y(\tau ; x, t), \tau) d \tau\right),
$$

where $y(\tau ; x, t)$ is the characteristic curve with speed $v^{*}$ through $(x, t)$. Therefore,

$$
\begin{aligned}
\varrho_{\max }-\varrho & =\varrho_{\max }-\varrho_{0}(y(0 ; x, t))+\varrho_{0}(y(0 ; x, t))\left(1-\exp \left(-\int_{0}^{t} \operatorname{div} v^{*}(y(\tau ; x, t), \tau) d \tau\right)\right) \\
& \geq \varrho_{\max }\left(\frac{1}{M_{0}}-\left|1-\exp \left(-\int_{0}^{t} \operatorname{div} v^{*}(y(\tau ; x, t), \tau) d \tau\right)\right|\right) .
\end{aligned}
$$


Use of $\left|1-e^{b}\right| \leq e^{|b|}|b|$ allows to bound $\left|1-\exp \left(-\int_{0}^{t} \operatorname{div} v^{*}(y(\tau ; \cdot), \tau) d \tau\right)\right| \leq \exp \left(\left\|\operatorname{div} v^{*}\right\|_{L^{\infty, 1}\left(Q_{t}\right)}\right)\left\|\operatorname{div} v^{*}\right\|_{L^{\infty, 1}\left(Q_{t}\right)}$. Owing to the continuity of $W^{1, p}(\Omega) \subset L^{\infty}(\Omega)$ and Hölder's inequality

$$
\left\|\operatorname{div} v^{*}\right\|_{L^{\infty, 1}\left(Q_{t}\right)} \leq c_{\Omega} \int_{0}^{t}\left\|\operatorname{div} v^{*}(\tau)\right\|_{W^{1, p}(\Omega)} d \tau \leq c_{\Omega} t^{1-\frac{1}{p}}\left\|v^{*}\right\|_{W_{p}^{2,0}\left(Q_{t}\right)} .
$$

Thus $1-\varrho / \varrho_{\max } \geq 1 / M_{0}-c_{\Omega} t^{1-\frac{1}{p}} \mathscr{V}\left(t ; v^{*}\right) \exp \left(c_{\Omega} t^{1-\frac{1}{p}} \mathscr{V}\left(t ; v^{*}\right)\right)$. Thanks to a similar argument applied to $\varrho_{\min }-\varrho$, we find that

$$
M(t) \leq \frac{M_{0}}{1-c_{\Omega} M_{0} t^{1-\frac{1}{p}} \mathscr{V}\left(t ; v^{*}\right) e^{c_{\Omega} t^{1-\frac{1}{p}} \mathscr{V}\left(t ; v^{*}\right)}}
$$

and define the function $\Psi_{3}$ to be the right-hand of the latter relation.

\section{The continuity estimate for $\mathcal{T}$}

We now want to combine the Propositions 11 and 12 with the linearisation of the continuity equation in Proposition 13 to study the fixed point map $\mathcal{T}$ described at the beginning of Sect. 6 and defined by the equations (46), (47), (48), (49) for given $v^{*} \in W_{p}^{2,1}\left(Q_{T} ; \mathbb{R}^{3}\right)$ and $q^{*} \in W_{p}^{2,1}\left(Q_{T} ; \mathbb{R}^{N-2}\right)$. We define $\mathscr{V}^{*}(t):=\mathscr{V}\left(t ; q^{*}\right)+$ $\mathscr{V}\left(t ; v^{*}\right)$. At first we state estimates for the lower-order nonlinearities (43), (45).

Lemma 14. For $u^{*}=\left(q^{*}, \zeta^{*}, \varrho^{*}, v^{*}\right) \in \mathcal{X}_{T, I}$, define $g^{*}:=g\left(x, t, u^{*}, D_{x}^{1} u^{*}\right)$ and, similarly, $f^{*}:=f\left(x, t, u^{*}, D_{x}^{1} u^{*}\right)$ via (43) and (45). There are continuous $\Psi_{g}, \Psi_{f}=\Psi\left(t, a_{1}, \ldots, a_{4}\right)$ defined for all $t \geq 0$ and $a_{1}, \ldots, a_{4} \geq 0$ such that

$$
\begin{aligned}
& \left\|g^{*}\right\|_{L^{p}\left(Q_{t}\right)} \leq \Psi_{g}\left(t, M^{*}(t),\left\|\left(q^{*}(0), v^{*}(0)\right)\right\|_{W_{p}^{2-2 / p}(\Omega)},\left\|\nabla \varrho^{*}\right\|_{L^{p, \infty}\left(Q_{t}\right)}, \mathscr{V}^{*}(t)\right), \\
& \left\|f^{*}\right\|_{L^{p}\left(Q_{t}\right)} \leq \Psi_{f}\left(t, M^{*}(t),\left\|\left(q^{*}(0), v^{*}(0)\right)\right\|_{W_{p}^{2-2 / p}(\Omega)},\left\|\nabla \varrho^{*}\right\|_{L^{p, \infty}\left(Q_{t}\right)}, \mathscr{V}^{*}(t)\right) .
\end{aligned}
$$

$\Psi_{g}$ and $\Psi_{f}$ are increasing in all arguments with $\Psi_{g}\left(0, a_{1}, \ldots, a_{4}\right)=0=\Psi_{f}\left(0, a_{1}\right.$, $\left.\ldots, a_{4}\right)$.

These estimates were proved in [5] for the case that the nonlinear coefficients $R, \tilde{M}$ are defined for $\varrho^{*}$ taking values in $] 0,+\infty\left[\right.$. The proof is exactly the same for $\varrho^{*}$ taking values in $I$, provided that we adapt the definition of $m^{*}(t), M^{*}(t)$ via (69). Moreover, the arguments are very similar to the ones used to bound the right-hand vector field $h$. This statement, that we next prove in detail, might serve as an illustration.

Lemma 15. Consider $u^{*}=\left(q^{*}, \zeta^{*}, \varrho^{*}, v^{*}\right) \in \mathcal{X}_{T, I}$. Define $h^{*}:=h\left(x, t, u^{*}\right)$ via (44). Then there is a continuous function $\Psi_{h}=\Psi_{h}\left(t, a_{1}, \ldots, a_{4}\right)$ defined for all $t \geq 0$ and $a_{1}, \ldots, a_{4} \geq 0$ such that

$$
\left\|h^{*}\right\|_{W_{p}^{1,0}\left(Q_{t}\right)} \leq \Psi_{h}\left(t, M^{*}(t),\left\|q^{*}(0)\right\|_{W_{p}^{2-2 / p}(\Omega)},\left\|\nabla \varrho^{*}\right\|_{L^{p, \infty}\left(Q_{t}\right)}, \mathscr{V}^{*}(t)\right) .
$$

The function $\Psi_{h}$ is increasing in all arguments. Moreover $\Psi_{h}\left(0, a_{1}, \ldots, a_{4}\right)=0$. 
Proof. Recall that $h:=A\left(\varrho^{*}, q^{*}\right) \tilde{b}(x, t)+d\left(\varrho^{*}, q^{*}\right) \hat{b}(x, t)$. With $c_{1}^{*}$ as in the proof of Prop. 11, we bound $\left|d\left(\varrho^{*}, q^{*}\right) \hat{b}\right| \leq c_{1}^{*}|\hat{b}|$ and $\left|A\left(\varrho^{*}, q^{*}\right) \tilde{b}\right| \leq c_{1}^{*}|\tilde{b}|$. Hence $\|h\|_{L^{p}\left(Q_{t}\right)} \leq c_{1}^{*}\left(\|\tilde{b}\|_{L^{p}\left(Q_{t}\right)}+\|\hat{b}\|_{L^{p}\left(Q_{t}\right)}\right)$. Lemma 27 allows to bound $\left\|q^{*}\right\|_{L^{\infty}\left(Q_{t}\right)} \leq$ $\left\|q^{0}\right\|_{L^{\infty}(\Omega)}+t^{\gamma} \mathscr{V}^{*}(t)$ and, evidently, $\left\|q^{0}\right\|_{L^{\infty}(\Omega)} \leq C\left\|q^{0}\right\|_{W_{p}^{2-2 / p}(\Omega)}$.

We then define a function $\Psi_{h}^{1}\left(t, a_{1}, \ldots, a_{4}\right):=\left(\|\tilde{b}\|_{L^{p}\left(Q_{t}\right)}+\|\hat{b}\|_{L^{p}\left(Q_{t}\right)}\right) c_{1}\left(a_{1}, a_{2}+\right.$ $\left.t^{\gamma} a_{4}\right)$. We see that $\Psi_{h}^{1}$ satisfies $\Psi_{h}^{1}\left(0, a_{1}, \ldots, a_{4}\right)=0$, and $\|h\|_{L^{p}\left(Q_{t}\right)} \leq \Psi_{h, t}^{1}$.

We compute $h_{x}^{*}$, and readily show a bound $\left|h_{x}^{*}\right| \leq c_{1}^{*}\left(\left(\left|\varrho_{x}^{*}\right|+\left|q_{x}^{*}\right|\right)(|\tilde{b}|+|\hat{b}|)+\right.$ $\left.\left|\tilde{b}_{x}\right|+\left|\hat{b}_{x}\right|\right)$. Hence

$$
\begin{aligned}
& \left\|h_{x}^{*}\right\|_{L^{p}\left(Q_{t}\right)} \\
& \leq c_{1}^{*}\left(\left\|\varrho_{x}^{*}\right\|_{L^{p, \infty}\left(Q_{t}\right)}+\left\|q_{x}^{*}\right\|_{L^{p, \infty}\left(Q_{t}\right)}\right)\left(\|\tilde{b}\|_{L^{\infty, p}\left(Q_{t}\right)}+\|\hat{b}\|_{L^{\infty, p}\left(Q_{t}\right)}\right) \\
& +c_{1}^{*}\left(\left\|\tilde{b}_{x}\right\|_{L^{p}\left(Q_{t}\right)}+\left\|\hat{b}_{x}\right\|_{L^{p}\left(Q_{t}\right)}\right) \\
& \leq c_{1}^{*}\left[\left(\|\tilde{b}\|_{L^{\infty, p}\left(Q_{t}\right)}+\|\hat{b}\|_{L^{\infty, p}\left(Q_{t}\right)}\right)\left(\left\|\varrho_{x}^{*}\right\|_{L^{p, \infty}\left(Q_{t}\right)}+\mathscr{V}^{*}(t)\right)+\left\|\tilde{b}_{x}\right\|_{L^{p}\left(Q_{t}\right)}\right. \\
& \left.\quad+\left\|\hat{b}_{x}\right\|_{L^{p}\left(Q_{t}\right)}\right]=: \Psi_{h}^{2} .
\end{aligned}
$$

We use again Lemma 27 to control $c_{1}^{*}$, seeing thus that the function $\Psi_{h}^{2}$ also possesses the desired structure $\left(\Psi_{h}^{2}=0\right)$.

We are now ready to establish the final estimate that allows to obtain the selfmapping property.

Proposition 16. For $\left(q^{*}, v^{*}\right) \in \mathcal{Y}_{T}$, the solution $(q, v)=\mathcal{T}\left(q^{*}, v^{*}\right)$ to the equations (46), (47), (48), (49) exists and is unique in the class $\mathcal{Y}_{t}$ for all $t$ subject to

$$
c M_{0} t^{1-\frac{1}{p}} \mathscr{V}^{*}(t) e^{c t^{1-\frac{1}{p}} \mathscr{V}^{*}(t)}<1
$$

where $c=c(\Omega)$ and $M_{0}$ are the same as in Prop. 13. There is a continuous function $\Psi_{6}=\Psi_{6}\left(t, a_{1}, \ldots, a_{4}\right)$ defined for all $t \geq 0$ and $a_{1} \ldots a_{4} \geq 0$ subject to the restriction

$$
c a_{1} t^{1-\frac{1}{p}} a_{4} e^{c t^{1-\frac{1}{p}} a_{4}}<1
$$

such that $\mathscr{V}(t ; q)+\mathscr{V}(t ; v) \leq \Psi_{6}\left(t, M_{0},\left\|\left(q^{0}, v^{0}\right)\right\|_{W_{p}^{2-2 / p}(\Omega)},\left\|\nabla \varrho_{0}\right\|_{L^{p}(\Omega)}, \mathscr{V}^{*}(t)\right)$. The function $\Psi_{6}$ is increasing in all arguments and

$$
\begin{aligned}
\Psi_{6}\left(0, M_{0},\left\|\left(q^{0}, v^{0}\right)\right\|_{W_{p}^{2-2 / p}(\Omega)}, \|\right. & \left.\nabla \varrho_{0} \|_{L^{p}(\Omega)}, \eta\right) \\
& =\Psi_{6}^{0}\left(M_{0},\left\|\left(q^{0}, v^{0}\right)\right\|_{W_{p}^{2-2 / p}(\Omega)},\left\|\nabla \varrho_{0}\right\|_{L^{p}(\Omega)}\right)
\end{aligned}
$$

for all $\eta>0$.

Proof. Applying Prop. 13, we first find the global solution $\varrho$ to the continuity equation (46) with data $v^{*}$ on $[0, T]$. The number $M(t)$ expressing the distance of the solution $\varrho$ to the thresholds $\left\{\varrho_{\min }, \varrho_{\max }\right\}$ remains finite for all $t$ subject to the restriction (90) (see Prop. 13). On this time interval, we can therefore insert $\left(\varrho, q^{*}\right)$ into the coefficients 
of the system (47), (48). Applying Prop. 11, we find a unique solution $(q, \zeta) \in$ $W_{p}^{2,1}\left(Q_{t} ; \mathbb{R}^{N-2}\right) \times W_{p}^{2,0}\left(Q_{t}\right)$. We then use $\left(\varrho, q^{*}\right)$ and $\zeta$ as data of the system (49). Applying Proposition 12, we obtain a solution $v \in W_{p}^{2,1}\left(Q_{t} ; \mathbb{R}^{3}\right)$ for all $t$ subject to (90). This shows that $(q, v):=\mathcal{T}\left(q^{*}, v^{*}\right)$ is well defined in $\mathcal{Y}_{t}$ for all $t$ subject to (90).

In order to verify the estimates, we first recall the outcome of Proposition 11 applied with $\varrho^{*}=\varrho$. It follows that

$$
\begin{aligned}
& \mathscr{V}(t ; q)+\|\zeta\|_{W_{p}^{2,0}\left(Q_{t}\right)} \\
& \leq C \Psi_{1}\left(t, M(t),\left\|q^{*}(0)\right\|_{C^{\beta}(\Omega)}, \mathscr{V}\left(t ; q^{*}\right),[\varrho]_{C^{\beta, \frac{\beta}{2}}\left(Q_{t}\right)},\|\nabla \varrho\|_{L^{p, \infty}\left(Q_{t}\right)}\right) \times \\
& \quad \times\left(1+[\varrho]_{C^{\beta, \frac{\beta}{2}}\left(Q_{t}\right)}\right)^{\frac{2}{\beta}}\left(\left\|q^{0}\right\|_{W_{p}^{2-2 / p}(\Omega)}+\left\|g^{*}\right\|_{L^{p}\left(Q_{t}\right)}+\left\|h^{*}+v^{*}\right\|_{W_{p}^{1,0}\left(Q_{t}\right)}\right) \\
& +C \Phi\left(t, M(t),\left\|q^{*}(0)\right\|_{C^{\beta}(\Omega)}, \mathscr{V}\left(t ; q^{*}\right),[\varrho]_{C^{\beta, \frac{\beta}{2}}\left(Q_{t}\right)},\|\nabla \varrho\|_{L^{p, \infty}\left(Q_{t}\right)}\left\|h^{*}+v^{*}\right\|_{L^{p}\left(Q_{t}\right)} .\right.
\end{aligned}
$$

Evidently $\left\|v^{*}\right\|_{W_{p}^{1,0}\left(Q_{t}\right)} \leq t^{\frac{1}{p}} \sup _{\tau \leq t}\|v(\tau)\|_{W^{1, p}(\Omega)} \leq t^{\frac{1}{p}} \mathscr{V}\left(t ; v^{*}\right)$. For the choices $\varrho^{*}=\varrho$ and $\beta:=1-3 / p$, Proposition 13 yields

$$
\begin{aligned}
M(t) & \leq \Psi_{3}\left(t, M_{0}, \mathscr{V}\left(t ; v^{*}\right)\right)=: \Psi_{3}(t, \ldots), \\
\|\nabla \varrho\|_{L^{p, \infty}\left(Q_{t}\right)} & \leq \Psi_{4}\left(t,\left\|\nabla \varrho_{0}\right\|_{L^{p}(\Omega)}, \mathscr{V}\left(t ; v^{*}\right)\right)=: \Psi_{4}(t, \ldots), \\
{[\varrho]_{C^{\beta, \frac{\beta}{2}}\left(Q_{t}\right)} } & \leq \Psi_{5}\left(t,\left\|\nabla \varrho_{0}\right\|_{L^{p}(\Omega)}, \mathscr{V}\left(t ; v^{*}\right)\right)=: \Psi_{5}(t, \ldots) .
\end{aligned}
$$

Moreover, due to the Lemma 14 and due to Lemma 15,

$$
\begin{aligned}
\left\|g^{*}\right\|_{L^{p}\left(Q_{t}\right)} & \leq \Psi_{g}\left(t, \Psi_{3}(t, \ldots),\left\|\left(q^{0}, v^{0}\right)\right\|_{W_{p}^{2-2 / p}(\Omega)}, \Psi_{4}(t, \ldots), \mathscr{V}^{*}(t)\right)=: \Psi_{g}(t, \ldots), \\
\left\|h^{*}\right\|_{W_{p}^{1,0}\left(Q_{t}\right)} & \leq \Psi_{h}\left(t, \Psi_{3}(t, \ldots),\left\|q^{0}\right\|_{W_{p}^{2-2 / p}(\Omega)}, \Psi_{4}(t, \ldots), \mathscr{V}^{*}(t)\right)=: \Psi_{h}(t, \ldots) .
\end{aligned}
$$

Combining all these estimates we can bound the quantity $\mathscr{V}(t ; q)+\|\zeta\|_{W_{p}^{2,0}\left(Q_{t}\right)}$ by some independent constant times the function

$$
\begin{aligned}
\Psi_{6}^{1}:= & \Psi_{1}\left(t, \Psi_{3}(t, \ldots),\left\|q^{0}\right\|_{C^{\beta}(\Omega)}, \mathscr{V}\left(t ; q^{*}\right), \Psi_{5}(t, \ldots), \Psi_{4}(t, \ldots)\right) \times \\
& \times\left(1+\Psi_{5}(t, \ldots)\right)^{\frac{2}{\beta}}\left(\left\|q^{0}\right\|_{W_{p}^{2-2 / p}(\Omega)}+\Psi_{g}(t, \ldots)+\Psi_{h}(t, \ldots)+t^{\frac{1}{p}} \mathscr{V}\left(t ; v^{*}\right)\right) \\
& +\Phi\left(t, \Psi_{3}(t, \ldots),\left\|q^{0}\right\|_{C^{\beta}(\Omega)}, \mathscr{V}\left(t ; q^{*}\right), \Psi_{5}(t, \ldots), \Psi_{4}(t, \ldots)\right)\left(\Psi_{h}(t, \ldots)+t^{\frac{1}{p}} \mathscr{V}\left(t ; v^{*}\right)\right) .
\end{aligned}
$$

Applying the inequalities $\mathscr{V}\left(t ; v^{*}\right), \mathscr{V}\left(t ; q^{*}\right) \leq \mathscr{V}^{*}(t)$, and $\left\|q^{0}\right\|_{C^{\beta}(\Omega)}$ $\leq c\left\|q^{0}\right\|_{W_{p}^{2-2 / p}(\Omega)}$, we reinterpret the latter expression as a function $\Psi_{6}^{1}$ of the arguments $t, M_{0},\left\|\left(q^{0}, v^{0}\right)\right\|_{W_{p}^{2-2 / p}(\Omega)},\left\|\nabla \varrho_{0}\right\|_{L^{p}(\Omega)}$ and $\mathscr{V}^{*}(t)$.

At $t=0$, we can use the estimates proved in the Propositions 11, 12 and the Prop. 13. Recall in particular that $\Psi_{1}\left(0, a_{1}, \ldots, a_{4}\right)=\Psi_{1}^{0}\left(a_{1}, a_{2}\right)$. Moreover, $\Psi_{3}\left(0, M_{0}, a_{4}\right)=$ 
$M_{0}$ (cf. (89)). Thus, since $\Psi_{5}\left(0, a_{1}, a_{4}\right) \leq C a_{1}$ is bounded independently of $a_{4}$, since $\Psi_{g}(0, \ldots)=0=\Psi_{h}(0, \ldots)$ (see Lemma 14,15$)$, we can compute that

$$
\begin{aligned}
& \Psi_{6}^{1}\left(0, M_{0},\left\|\left(q^{0}, v^{0}\right)\right\|_{W_{p}^{2-2 / p}(\Omega)},\left\|\nabla \varrho_{0}\right\|_{L^{p}(\Omega)}, \mathscr{V}^{*}(t)\right) \\
& \quad=\Psi_{1}^{0}\left(M_{0},\left\|q^{0}\right\|_{C^{1-3 / p}(\Omega)}\right)\left(1+\left\|\nabla \varrho_{0}\right\|_{L^{p}(\Omega)}\right)^{\frac{2 p}{p-3}}\left\|q^{0}\right\|_{W_{p}^{2-2 / p}(\Omega)} .
\end{aligned}
$$

We next apply Proposition 12 with $\varrho^{*}=\varrho$ and $f=f^{*}$ to obtain

$$
\begin{aligned}
\mathscr{V}(t ; v) & \leq C \Psi_{2}\left(t, \sup _{\tau \leq t}[\varrho(\tau)]_{C^{\alpha}(\Omega)}\right)\left(1+\sup _{\tau \leq t}[\varrho(\tau)]_{C^{\alpha}(\Omega)}\right)^{\frac{2}{\alpha}}\left(\left\|v^{0}\right\|_{W_{p}^{2-2 / p}(\Omega)}\right. \\
& \left.+\left\|f^{*}\right\|_{L^{p}\left(Q_{t}\right)}+\|\nabla \zeta\|_{L^{p}\left(Q_{t}\right)}\right) .
\end{aligned}
$$

For $\alpha=1-3 / p$, the norm $\mathscr{V}(t ; v)$ is estimated above by the quantity

$$
\Psi_{2}\left(t, \Psi_{5}(t, \ldots)\right)\left(1+\Psi_{5}(t, \ldots)\right)^{\frac{2}{\alpha}}\left(\left\|v^{0}\right\|_{W_{p}^{2-2 / p}(\Omega)}+\Psi_{f}(t, \ldots)+\|\nabla \zeta\|_{L^{p}\left(Q_{t}\right)}\right) .
$$

Recalling that (92) and the subsequent arguments also provide an estimate for $\|\nabla \zeta\|_{L^{p}\left(Q_{t}\right)}$ by $\Psi_{6}^{1}$, we reinterpret the latter function as a $\Psi_{6}^{2}$ of the same arguments, and we note that

$$
\begin{aligned}
& \Psi_{6}^{2}\left(0, M_{0},\left\|\left(q^{0}, v^{0}\right)\right\|_{W_{p}^{2-2 / p}(\Omega)},\left\|\nabla \varrho_{0}\right\|_{L^{p}(\Omega)}, \mathscr{V}^{*}(t)\right) \\
& =\Psi_{2}^{0} \times\left(1+\left\|\nabla \varrho_{0}\right\|_{L^{p}(\Omega)}\right)^{\frac{2 p}{p-3}}\left(\left\|v^{0}\right\|_{W_{p}^{2-2 / p}(\Omega)}+\Psi_{6}^{1}(0, \ldots)\right) \\
& =\left(\frac{1}{\min \left\{1, \varrho_{\min }\right\}}\right)^{\frac{2 p}{p-3}}\left(\frac{\varrho_{\max }}{\varrho_{\min }}\right)^{\frac{p+1}{p}}\left(1+\left\|\nabla \varrho_{0}\right\|_{L^{p}(\Omega)}\right)^{\frac{2 p}{p-3}}\left(\left\|v^{0}\right\|_{W_{p}^{2-2 / p}(\Omega)}+\Psi_{6}^{1}(0, \ldots)\right) .
\end{aligned}
$$

The value of $\Psi_{6}^{1}(0, \ldots)$ is given in (93). We define $\Psi_{6}:=\Psi_{6}^{1}+\Psi_{6}^{2}$. Due to Proposition 13, the function $\Psi_{3}\left(t, M_{0}, \mathscr{V}\left(t ; v^{*}\right)\right)$ is finite for all arguments satisfying (91), and therefore $\Psi_{6}$ is finite under the same condition. The claim follows.

We sum up the continuity estimates in the following statement.

Proposition 17. We adopt the assumptions of Theorem 8. Given $\left(q^{*}, v^{*}\right) \in \mathcal{Y}_{T}$, we define a map $\mathcal{T}\left(q^{*}, v^{*}\right)=(q, v)$ via solution to the equations (46), (47), (48), (49) with homogeneous boundary conditions (31), (30) and initial conditions $\left(q^{0}, \varrho_{0}, v^{0}\right)$. Then, there are $0<T_{0} \leq T$ and $\eta_{0}>0$ depending on the data $R_{0}:=\left(M_{0}, \|\left(q^{0}, v^{0}\right)\right.$ $\left.\left\|_{W_{p}^{2-2 / p}(\Omega)},\right\| \nabla \varrho_{0} \|_{L^{p}(\Omega)}\right)$ such that $\mathcal{T}$ maps the ball with radius $\eta_{0}$ in $\mathcal{Y}_{T_{0}}$ into itself.

Proof. We define $a_{0}>0$ to be the solution to the equation $c M_{0} x e^{c x}=1$ associated with the numbers in (91). We apply the Lemma 9 with $\Psi\left(t, R_{0}, \eta\right):=\Psi_{6}\left(t, R_{0}, \eta\right)$ from Prop. 16, and the claim follows. 


\section{Proof of the theorem on short-time well-posedness}

\subsection{Existence and uniqueness}

We choose $T_{0}, \eta_{0}>0$ according to Proposition 17. Starting from $\left(q^{1}, v^{1}\right)=0$, we consider a fixed point iteration $\left(q^{n+1}, v^{n+1}\right):=\mathcal{T}\left(q^{n}, v^{n}\right)$ for $n \in \mathbb{N}$. Recalling (67), we define $\mathscr{V}^{n+1}(t):=\mathscr{V}\left(t ; q^{n+1}\right)+\mathscr{V}\left(t ; v^{n+1}\right)$. Since obviously $\mathscr{V}^{1}(t) \equiv 0$, Proposition 17 guarantees that

$$
\sup _{n \in \mathbb{N}} \mathscr{V}^{n}\left(T_{0}\right) \leq \eta_{0}, \quad \sup _{n \in \mathbb{N}}\left\|\varrho^{n}\right\|_{W_{p, \infty}^{1,1}\left(Q_{T_{0}}\right)}+\left\|\zeta^{n}\right\|_{W_{p}^{2,0}\left(Q_{T_{0}}\right)}<+\infty .
$$

From Lemma 18 hereafter, we infer that the fixed-point iteration therefore yields strongly convergent subsequences in $L^{2}\left(Q_{T_{0}}\right)$ for the components of $q^{n}, \zeta_{n}, \varrho_{n}$ and $v^{n}$ and for the gradients $q_{x}^{n}, \zeta_{x}^{n}$ and $v_{x}^{n}$. The passage to the limit in the approximation scheme is then a straightforward exercise, since we can rely on a uniform bound in $\mathcal{X}_{T_{0}}$. The proofs are almost identical with the fixed-point iteration in [5]. We leave the minor changes to the interested reader, and state without proof the following iteration lemma.

Lemma 18. For $n \in \mathbb{N}$, we define

$$
r^{n+1}:=q^{n+1}-q^{n}, \quad \chi^{n+1}:=\zeta^{n+1}-\zeta^{n}, \quad \sigma^{n+1}:=\varrho^{n+1}-\varrho^{n}, \quad w^{n+1}:=v^{n+1}-v^{n} .
$$

Then there are $k_{0}, p_{0}>0$ and $0<t_{1} \leq T_{0}$ such that for all $t \in\left[0, T_{0}-t_{1}\right]$, the quantity

$$
\begin{aligned}
E^{n+1}(t):= & k_{0} \sup _{\tau \in\left[t, t+t_{1}\right]}\left(\left\|r^{n+1}(\tau)\right\|_{L^{2}(\Omega)}^{2}+\left\|w^{n+1}(\tau)\right\|_{L^{2}(\Omega)}^{2}+\left\|\sigma_{n+1}\right\|_{L^{2}(\Omega)}^{2}\right) \\
& +p_{0} \int_{Q_{t, t+t_{1}}}\left(\left|\nabla r^{n+1}\right|^{2}+\left|\nabla \chi^{n+1}\right|^{2}+\left|\nabla w^{n+1}\right|^{2}\right) d x d \tau
\end{aligned}
$$

satisfies $E^{n+1}(t) \leq \frac{1}{2} E^{n}(t)$ for all $n \in \mathbb{N}$.

\subsection{Verification of continuation criteria}

In order to complete the proof of the Theorems 1, 2 it remains to investigate the claimed characterisations of the maximal existence interval.

Lemma 19. Suppose that $u=(q, \zeta, \varrho, v) \in \mathcal{X}_{t}$ is a solution to $\tilde{\mathscr{A}}(u)=0$ and $u(0)=u_{0}$ for all $0<t<T^{*}$. Then the two following statements are valid:

(1) If $\mathscr{N}(t):=\|q\|_{C^{\alpha, \alpha / 2}\left(Q_{t}\right)}+\|\nabla q\|_{L^{\infty, p}\left(Q_{t}\right)}+\|v\|_{L^{z} p, p}\left(Q_{t}\right)+\int_{0}^{t}[\nabla v(\tau)]_{C^{\alpha}(\Omega)} d \tau$ with $\alpha>0$ arbitrary and $z=z(p)$ defined in Theorem 1 , and $M(\varrho, t)(c f .(69))$ are finite for $t \nearrow T^{*}$, then it is possible to extend the solution to a larger time interval. 
(2) If the tensor $M$ occurring in (4) satisfies the additional conditions stated in Theorem 2 , and if $\mathcal{K}(t):=\|q\|_{W_{p}^{2,1}\left(Q_{t} ; \mathbb{R}^{N-2}\right)}+\|\zeta\|_{W_{p}^{2,0}\left(Q_{t}\right)}+\|v\|_{W_{p}^{2,1}\left(Q_{t} ; \mathbb{R}^{3}\right)}$ remains finite for $t \nearrow T^{*}$, then the solution can be extended without additional condition concerning $M(\varrho, t)$.

Proof. First criterion (1). We must show that the quantity $\mathscr{V}(t ; q)+\mathscr{V}(t ; v)$ is bounded by a continuous function of $t, M(\varrho, t), \mathcal{N}(t)$. We will only sketch this point, which relies on going carefully through the proofs of the estimates in the Propositions 11,12 in the spirit of [5].

To begin with, we notice that the components of $v_{x}$ have all spatial mean-value zero over $\Omega$ due to the boundary condition (30). Hence, for $\alpha>0$, inequalities $\left\|v_{x}(\tau)\right\|_{L^{\infty}(\Omega)} \leq c_{\Omega}\left[v_{x}(\tau)\right]_{C^{\alpha}(\Omega)}$ and $\left\|v_{x}\right\|_{L^{\infty, 1}\left(Q_{t}\right)} \leq c_{\Omega} \int_{0}^{t}\left[v_{x}(\tau)\right]_{C^{\alpha}(\Omega)} d \tau$ are available. For the solution to the continuity equation, Theorem 2 of [29] (see also Proposition 7.7 in [5]) implies that $\sup _{\tau<t}[\varrho(\tau)]_{C^{\alpha}(\Omega)}$ is bounded by a function of $\int_{0}^{t}\left[v_{x}(\tau)\right]_{C^{\alpha}(\Omega)} d \tau$, thus also by a function of $\mathcal{N}(t)$. Moreover, as in the same references, we show for all $t \geq 0$ that

$$
\begin{aligned}
\left\|\varrho_{x}(t)\right\|_{L^{p}(\Omega)} & \leq \phi\left(R_{0},\left\|v_{x}\right\|_{L^{\infty, 1}\left(Q_{t}\right)}\right)\left(1+\int_{0}^{t}\left\|v_{x, x}(\tau)\right\|_{L^{p}(\Omega)} d \tau\right) \\
& \leq \phi\left(R_{0}, \mathcal{N}(t)\right)(1+\mathscr{V}(t ; v)) .
\end{aligned}
$$

Here and throughout the proof, we denote by $\phi$ some generic continuous function increasing in its arguments, and $R_{0}$ stands for the initial data and the external forces.

We next exploit the momentum balance equation for $v$. We apply Proposition 12, hence $\mathscr{V}(t ; v) \leq \phi(t, \mathcal{N}(t))\left(\|f\|_{L^{p}\left(Q_{t}\right)}+\|\nabla \zeta\|_{L^{p}\left(Q_{t}\right)}+\left\|v^{0}\right\|_{W_{p}^{2-2 / p}(\Omega)}\right)$. The function $f$ obeys (45) and therefore

$$
\begin{aligned}
|f(x, t)| \leq & |\nabla \varrho(x, t)| \sup _{Q_{t}}\left|P_{\varrho}(\varrho, q)\right|+|\nabla q(x, t)| \sup _{Q_{t}}\left|P_{q}(\varrho, q)\right| \\
& +c(|v(x, t)||\nabla v(x, t)|+|\bar{b}(x, t)|+|\tilde{b}(x, t)|) \sup _{Q_{t}} \varrho+|\hat{b}(x, t)| .
\end{aligned}
$$

Coefficients depending on $\varrho$ and $q$ can in general be bounded following the example of

$$
\sup _{Q_{t}}\left|P_{\varrho}(\varrho, q)\right| \leq \phi\left(M(\varrho, t),\|q\|_{L^{\infty}\left(Q_{t}\right)} \leq \phi(M(\varrho, t), \mathcal{N}(t)) .\right.
$$

Therefore, we show that

$$
\begin{aligned}
\|f\|_{L^{p}\left(Q_{t}\right)}^{p} \leq \phi(M(\varrho, t), \mathcal{N}(t))( & \|\nabla \varrho\|_{L^{p}\left(Q_{t}\right)}^{p}+\|\nabla q\|_{L^{p}\left(Q_{t}\right)}^{p}+\|v \nabla v\|_{L^{p}\left(Q_{t}\right)}^{p} \\
& \left.+\|\tilde{b}\|_{L^{p}\left(Q_{t}\right)}^{p}+\|\bar{b}\|_{L^{p}\left(Q_{t}\right)}^{p}+\|\hat{b}\|_{L^{p}\left(Q_{t}\right)}^{p}\right) .
\end{aligned}
$$

We define $A_{0}(t):=\|\tilde{b}\|_{L^{p}\left(Q_{t}\right)}^{p}+\|\bar{b}\|_{L^{p}\left(Q_{t}\right)}^{p}+\|\hat{b}\|_{L^{p}\left(Q_{t}\right)}^{p}+\left\|v^{0}\right\|_{W_{p}^{2-2 / p}(\Omega)}$, hence

$$
\begin{gathered}
\mathscr{V}^{p}(t ; v) \leq \phi(M(\varrho, t), \mathcal{N}(t))\left(\|\nabla \zeta\|_{L^{p}\left(Q_{t}\right)}^{p}+\|\nabla \varrho\|_{L^{p}\left(Q_{t}\right)}^{p}+\|v \nabla v\|_{L^{p}\left(Q_{t}\right)}^{p}\right. \\
\left.+\|\nabla q\|_{L^{p}\left(Q_{t}\right)}^{p}+A_{0}(t)\right) .
\end{gathered}
$$


As shown, $\|\nabla \varrho\|_{L^{p}\left(Q_{t}\right)}^{p} \leq \phi\left(R_{0},\left\|v_{x}\right\|_{L^{\infty, 1}\left(Q_{t}\right)}\right) \int_{0}^{t}(1+\mathscr{V}(\tau ; v))^{p} d \tau$, and $\zeta$ satisfies the weak Neumann problem (48), hence

$$
\begin{aligned}
\|\nabla \zeta\|_{L^{p}\left(Q_{t}\right) \leq} & \phi(M(\varrho, t), \mathcal{N}(t))\left(\|\nabla q\|_{L^{p}\left(Q_{t}\right)}+\|v\|_{L^{p}\left(Q_{t}\right)}\right. \\
& \left.+\|\tilde{b}\|_{L^{p}\left(Q_{t}\right)}+\|\hat{b}\|_{L^{p}\left(Q_{t}\right)}\right) .
\end{aligned}
$$

We define $z=\frac{3}{p-2}$ if $3<p<5, z>1$ arbitrary if $p=5$ and $z=1$ if $p>5$. Recalling the continuity of the embedding $W_{p}^{1-2 / p} \subset L^{3 p /(5-p)^{+}}$, we show by means of Hölder's inequality that $\left\|v v_{x}\right\|_{L^{p}\left(Q_{t}\right)}^{p} \leq c_{\Omega} \int_{0}^{t}\|v(\tau)\|_{L^{z} p}^{p} \mathscr{V}^{p}(\tau ; v) d \tau$. Therefore, combining the latter bounds yields

$$
\begin{aligned}
& \mathscr{V}^{p}(t ; v) \leq \phi(t, M(\varrho, t), \mathcal{N}(t)) \\
& \quad \times\left(\int_{0}^{t}\left(1+\|v(\tau)\|_{L^{z} p}^{p}\right) \mathscr{V}^{p}(\tau ; v) d \tau+\|\nabla q\|_{L^{p}\left(Q_{t}\right)}^{p}+A_{0}(t)\right) .
\end{aligned}
$$

We invoke the Gronwall Lemma, hence $\mathscr{V}^{p}(t ; v) \leq \phi(t, M(\varrho, t)$, $\mathcal{N}(t))\left(\|\nabla q\|_{L^{p}\left(Q_{t}\right)}^{p}+A_{0}(t)\right)$. Since $\|\nabla q\|_{L^{p}\left(Q_{t}\right)}$ is also controlled by a function of $t$ and $\mathcal{N}(t)$, so does $\mathscr{V}^{p}(t ; v)$. It follows that $\|\nabla \varrho\|_{L^{p, \infty}\left(Q_{t}\right)}^{p} \leq \phi\left(t, R_{0}, \mathcal{N}(t)\right)$. For $\beta=1-3 / p$, the Proposition 13 yields that $\|\varrho\|_{C^{\beta, \beta / 2}\left(Q_{t}\right)} \leq \phi\left(t, R_{0}, \mathcal{N}(t)\right)$. Recalling that $q$ satisfies (76), we can now finish the proof as in [5], Lemma 9.2.

Second criterion (2). The more interesting point is to get rid of the dependence on the distance $M(\varrho, t)$ to the density thresholds in the estimates. First we note that the relation (28) implies for the gradient of the pressure

$$
\begin{aligned}
\nabla P(\varrho, q)=F:= & -\nabla \zeta-\varrho\left(\partial_{t} v+(v \cdot \nabla) v\right)+\operatorname{div} \mathbb{S}(\nabla v) \\
& +R(\varrho, q) \cdot \tilde{b}+\hat{b}+\varrho \bar{b} .
\end{aligned}
$$

Clearly, $\|F\|_{L^{p}\left(Q_{t}\right)}$ is bounded by a function of $b$ and the norms of $\zeta$ and $v$ occurring in the quantity $\mathcal{K}(t)$. We notice in particular that this function is independent on $M(\varrho, t)$.

In order to obtain a bound on the entire pressure gradient, we employ the continuity equation (27). We compute that

$$
\begin{aligned}
\partial_{t} P(\varrho, q) & =P_{\varrho}(\varrho, q) \partial_{t} \varrho+P_{q}(\varrho, q) \partial_{t} q \\
& =P_{\varrho}(\varrho, q)(-v \cdot \nabla \varrho-\varrho \operatorname{div} v)+P_{q}(\varrho, q) \partial_{t} q .
\end{aligned}
$$

Define $m(\varrho, t):=\min _{Q_{t}}\left\{1-\varrho / \varrho_{\max }, \varrho / \varrho_{\min }-1\right\}$. Thanks to Lemma 24, the properties of the pressure function guarantee that $\left|P_{\varrho}(\varrho, q)\right| \leq c_{4} m(\varrho, t)^{-1}$ in $Q_{t}$. Since $\left|P_{\varrho}(\varrho, q)\right||\nabla \varrho|=\left|\nabla P(\varrho, q)-P_{q}(\varrho, q) \nabla q\right|$, the same Lemma 24 also implies that

$$
\left.c_{3} m(\varrho, t)^{-1}|\nabla \varrho| \leq|\nabla P(\varrho, q)|+c_{5}|\nabla q|\right) \text {. }
$$

By these means, the time derivative of pressure is bounded via

$$
\begin{aligned}
\left|\partial_{t} P(\varrho, q)\right| & \leq c_{4} m^{-1}(|v||\nabla \varrho|+|\varrho||\operatorname{div} v|)+\left|P_{q}\right|\left|\partial_{t} q\right| \\
& \leq c_{4} \frac{1+c_{5}}{c_{3}}|v|(|\nabla P(\varrho, q)|+|\nabla q|)+c_{4} \varrho_{\max } m^{-1}|\operatorname{div} v|+c_{5}\left|\partial_{t} q\right| .
\end{aligned}
$$


We want to obtain a control on $m(\varrho, t)^{-1}|\operatorname{div} v|$. To this aim, we recall the relation (26), which allows us to compute

$$
\begin{aligned}
\operatorname{div} v & =\operatorname{div}(d(\varrho, q)(\nabla \zeta-\hat{b})+A(\varrho, q)(\nabla q-\tilde{b})) \\
= & d(\varrho, q) \operatorname{div}(\nabla \zeta-\hat{b})+A(\varrho, q) \operatorname{div}(\nabla q-\tilde{b})+(\nabla \zeta-\hat{b}) \cdot \nabla d(\varrho, q) \\
& +\nabla A(\varrho, q) \cdot(\nabla q-\tilde{b}) \\
= & d \operatorname{div}(\nabla \zeta-\hat{b})+A \operatorname{div}(\nabla q-\tilde{b})+\left[(\nabla \zeta-\hat{b}) d_{q}+(\nabla q-\tilde{b}) A_{q}\right] \nabla q \\
& +\nabla \varrho\left[d_{\varrho}(\nabla \zeta-\hat{b})+A_{\varrho}(\nabla q-\tilde{b})\right] .
\end{aligned}
$$

We recall Lemma 24, which shows for a constant $c_{1}$, depending only on the kinetic matrix $M$ and the free energy function $k$, that

$$
|d(\varrho, q)|+|A(\varrho, q)|+\left|d_{q}(\varrho, q)\right|+\left|A_{q}(\varrho, q)\right| \leq c_{1} m(\varrho)
$$

and, moreover, that $\left|d_{\varrho}(\varrho, q)\right|+\left|A_{\varrho}(\varrho, q)\right| \leq c_{2}$. Applying these estimates to (97), we obtain that

$$
\begin{aligned}
\frac{1}{m(\varrho, t)}|\operatorname{div} v| \leq & c_{1} \underbrace{\left[\left|D^{2} \zeta\right|+\left|D^{2} q\right|+\left|\tilde{b}_{x}\right|+\left|\hat{b}_{x}\right|+|\nabla q|(|\nabla \zeta|+|\nabla q|+|\tilde{b}|+|\hat{b}|)\right]}_{=: G} \\
& +c_{2} \frac{|\nabla \varrho|}{m(\varrho, t)}(|\nabla \zeta|+|\nabla q|+|\tilde{b}|+|\hat{b}|) .
\end{aligned}
$$

Recalling again that $m(\varrho)^{-1}|\nabla \varrho| \leq c(|\nabla P(\varrho, q)|+|\nabla q|)$, we get the bound

$$
\frac{1}{m(\varrho, t)}|\operatorname{div} v| \leq|G|+c(|\nabla P(\varrho, q)|+|\nabla q|)(|\nabla \zeta|+|\nabla q|+|\tilde{b}|+|\hat{b}|) .
$$

It is readily verified that $G$ is continuously bounded in $L^{p}\left(Q_{t}\right)$ by the quantity $\mathcal{K}(t)$, independently of $M(\varrho, t)$. Since $\left|\zeta_{x}\right|+\left|q_{x}\right|+|\tilde{b}|+|\hat{b}|$ is bounded in $L^{\infty, p}\left(Q_{t}\right)$, we recall (95) to finally obtain

$$
\left\|m(\varrho, t)^{-1} \operatorname{div} v\right\|_{L^{p, \frac{p}{2}}\left(Q_{t}\right)} \leq \Psi(t, \mathcal{K}(t)) .
$$

By means of (98), (95), (96) we see that also $\left\|\partial_{t} P(\varrho, q)\right\|_{L^{p, p / 2}(Q)}$ is bounded by a function of $t$ and $\mathcal{K}(t)$, independently of $M(\varrho, t)$. Overall we have $\left\|\partial_{x} P\right\|_{L^{p}\left(Q_{t}\right)}+$ $\left\|\partial_{t} P\right\|_{L^{p, p / 2}\left(Q_{t}\right)} \leq \Psi$. For $p>5$, we can show that this implies a bound $\|P\|_{L^{\infty}\left(Q_{t}\right)} \leq$ $C(t) \Psi$, where $C(t)$ is the embedding constant of an anisotropic Sobolev space into $L^{\infty}\left(Q_{t}\right)$. It remains to recall that for the choice (7), the function $P$ satisfies (cf. [14], Proposition 5.3)

$$
|P(\varrho, q)| \geq c \ln \max \left\{\frac{1}{\varrho_{\max }-\varrho}, \frac{1}{\varrho-\varrho_{\min }}\right\}-C(1+|q|) .
$$

This implies that $M(\varrho, t) \leq C_{1} e^{C_{2}\left(\|P(\varrho, q)\|_{L^{\infty}\left(Q_{t}\right)}+\|q\|_{L^{\infty}\left(Q_{t}\right)}\right)}$, and the claim follows. 


\section{Global well-posedness}

\subsection{The map $\mathcal{T}^{1}$ is well defined}

We consider the equations (59), (61), (62), (63), (64) characteristic of the definition of the map $\mathcal{T}^{1}$. We recall that these equations are obtained by comparing a solution to some suitable extension $\left(\hat{q}^{0}, \hat{v}^{0}\right) \in \mathcal{Y}_{T}$, to be constructed here below, of the initial data. The initial density $\varrho_{0}$ is extended by a function $\hat{\varrho}^{0}$ obtained via the solution of (50). We moreover introduce the function $\hat{\zeta}^{0}$, solution to (51).

In order to define $\mathcal{T}^{1}$ we must make sense of the linear operators $\left(g^{1}\right)^{\prime}\left(u^{*}, \hat{u}^{0}\right)$, $\left(h^{1}\right)^{\prime}\left(u^{*}, \hat{u}^{0}\right)$ and $\left(f^{1}\right)^{\prime}\left(u^{*}, \hat{u}^{0}\right)$. The density components in the vectors $\hat{u}^{0}=\left(\hat{q}^{0}, 1\right.$, $\hat{\varrho}^{0}, \hat{v}^{0}$ ) and $u^{*}$ (def. in (60)) must therefore assume values in $I$ up to time $T>0$ ! This property is to be expected if the initial data are close enough to an equilibrium solution $\left(\rho^{\mathrm{eq}}, p^{\mathrm{eq}}, v^{\mathrm{eq}}\right)$ defined by the relations (16), (17). The distance of the initial data to this solution is expressed by the number

$$
R_{1}:=\left\|q^{0}-q^{\mathrm{eq}}\right\|_{W_{p}^{2-2 / p}\left(\Omega ; \mathbb{R}^{N-2}\right)}+\left\|v^{0}-v^{\mathrm{eq}}\right\|_{W_{p}^{2-2 / p}\left(\Omega ; \mathbb{R}^{3}\right)}+\left\|\varrho^{0}-\varrho^{\mathrm{eq}}\right\|_{W^{1, p}(\Omega)},
$$

in which $\varrho^{\mathrm{eq}}:=\sum_{i=1}^{N} \rho_{i}^{\mathrm{eq}}$ and $q_{\ell}^{\mathrm{eq}}=\eta^{\ell} \cdot \nabla_{\rho} k\left(\rho^{\mathrm{eq}}\right)$ for $\ell=1, \ldots, N-2$. Throughout this section, we moreover employ the abbreviation

$$
\left.R_{0}:=\left\|\hat{u}^{0}\right\| \mathcal{X}_{T}+\left\|\hat{\varrho}^{0}\right\|_{W_{p}^{2,0}\left(Q_{T}\right)}+\|\tilde{b}\|_{W_{p}^{1,0}\left(Q_{T}\right)}+\|\hat{b}\|_{W_{p}^{1,0}\left(Q_{T}\right)}+\|\bar{b}\|_{L^{p}\left(Q_{T}\right)}\right) .
$$

Observe the occurrence of the higher-order $W_{p}^{2,0}$-norm of $\hat{\varrho}^{0}$ in the definition of $R_{0}$.

To commence with, we recall a result of [5] for estimating the gradient of solutions to a perturbed continuity equation. The proof in [5] is given for zero initial conditions, but the extension to the nonzero case is completely straightforward.

Lemma 20. Assume that $\sigma \in W_{p, \infty}^{1,1}\left(Q_{T}\right)$ satisfies $\partial_{t} \sigma+\operatorname{div}(\sigma v)=-\operatorname{div}\left(\hat{\varrho}^{0} w\right)$ with $\hat{\varrho}^{0} \in W_{p, \infty}^{1,1}\left(Q_{T}\right) \cap W_{p}^{2,0}\left(Q_{T}\right)$ and $v, w \in W_{p}^{2,1}\left(Q_{T} ; \mathbb{R}^{3}\right)$. Then there are constants $C, c$, depending only on $\Omega$, such that

$$
\begin{aligned}
\|\sigma(t)\|_{W^{1, p}(\Omega)}^{p} \leq & C \exp \left(c \int_{0}^{t}\left[\left\|v_{x}(\tau)\right\|_{L^{\infty}(\Omega)}+\left\|v_{x, x}(\tau)\right\|_{L^{p}(\Omega)}+1\right] d \tau\right) \times \\
\times & \left(\|\sigma(0)\|_{W^{1, p}(\Omega)}^{p}+\left\|\hat{\varrho}^{0}\right\|_{W_{p}^{2,0}\left(Q_{t}\right)}^{p}\|w\|_{L^{\infty}\left(Q_{t}\right)}^{p}\right. \\
& \left.+\left\|\hat{\varrho}^{0}\right\|_{W_{p, \infty}^{1,1}\left(Q_{t}\right)}^{p}\|w\|_{W_{p}^{2,0}\left(Q_{t}\right)}^{p}\right)
\end{aligned}
$$

for all $t \leq T$.

Construction of global extensions. Under the assumptions of Theorem 3 , the trivial extensions $q^{\mathrm{eq}}(x, t):=q^{\mathrm{eq}}(x)$ and $v^{\mathrm{eq}}(x, t):=v^{\mathrm{eq}}(x)$ are such that $q^{\mathrm{eq}} \in W_{p, \infty}^{2, \infty}$ 
and $v^{\mathrm{eq}} \in W_{p, \infty}^{3, \infty}\left(Q_{T}\right)$. Introduce on $\Omega$ the differences $q^{1}(x):=q^{0}(x)-q^{\mathrm{eq}}(x)$ and $v^{1}(x):=v^{0}(x)-v^{\mathrm{eq}}(x)$. We extend $q^{0}$ and $v^{0}$ via

$$
\hat{v}^{0}(x, t):=v^{\mathrm{eq}}(x)+\underbrace{\mathcal{E}\left(v^{1}\right)(x, t)}_{=: \hat{v}^{1}(x, t)}, \quad \hat{q}^{0}(x, t):=q^{\mathrm{eq}}(x)+\underbrace{\mathcal{E}\left(q^{1}\right)(x, t)}_{=: \hat{q}^{1}(x, t)},
$$

in which $\mathcal{E}: W_{p}^{2-2 / p}(\Omega) \rightarrow W_{p}^{2,1}\left(Q_{T}\right)$ is a linear, bounded extension operator. Typically, the components of $q^{1}, v^{1}$ defined in $\Omega$ are first extended to elements of $W_{p}^{2-2 / p}\left(\mathbb{R}^{3}\right)$ with bounded support. Then, we solve Cauchy-problems for the heat equation to extend the functions into $\mathbb{R}^{3} \times[0, T]$ or even $\mathbb{R}^{4}$. As the assumptions in Theorem 3 moreover guarantee that $v^{1} \in W^{2, p}(\Omega)$, this procedure even yields the additional regularity $\hat{v}^{1} \in W_{p}^{4,2}\left(Q_{T} ; \mathbb{R}^{3}\right)$ (cf. [21], Ch. 4, Par. 3, inequality (3.3)).

Then, the extensions defined in (101) satisfy

$$
\begin{aligned}
\left\|\hat{q}^{0}-q^{\mathrm{eq}}\right\|_{W_{p}^{2,1}\left(Q_{T}\right)}+\left\|\hat{v}^{0}-v^{\mathrm{eq}}\right\|_{W_{p}^{2,1}\left(Q_{T}\right)} & \leq C_{\mathcal{E}}\left(\left\|q^{1}\right\|_{W_{p}^{2-2 / p}(\Omega)}+\left\|v^{1}\right\|_{W_{p}^{2-2 / p}(\Omega)}\right) \\
& \leq C_{\mathcal{E}} R_{1}, \\
\left\|\hat{v}^{0}\right\|_{W_{p}^{3,0}\left(Q_{T}\right)} & \leq C\left(\left\|v^{\mathrm{eq}}\right\|_{W^{3, p}(\Omega)}+\left\|v^{0}\right\|_{W^{2, p}(\Omega)}\right) .
\end{aligned}
$$

In order to extend $\varrho^{0}$, we solve $\partial_{t} \hat{\varrho}^{0}+\operatorname{div}\left(\hat{\varrho}^{0} \hat{v}^{0}\right)=0$ with initial condition $\hat{\varrho}^{0}=\varrho^{0}$. By these means, $\hat{\varrho}^{0} \in W_{p, \infty}^{1,1}\left(Q_{T}\right)$. Due to (103), we can even show that $\hat{\varrho}^{0} \in W_{p}^{2,0}\left(Q_{T}\right)$. We next extend the equilibrium solution via $\varrho^{\mathrm{eq}}(x, t):=\varrho^{\mathrm{eq}}(x) \in W_{p, \infty}^{2, \infty}\left(Q_{T}\right)$. Then, by definition, $\operatorname{div}\left(\hat{\varrho}^{\mathrm{eq}} \hat{v}^{\mathrm{eq}}\right)=0$ in $Q_{T}(\mathrm{cp} .(16))$, and $\partial_{t} \hat{\varrho}^{\mathrm{eq}}=0$. Thus, the difference $\hat{\varrho}^{1}:=\hat{\varrho}^{0}-\varrho^{\text {eq }}$ is a solution to

$$
\partial_{t} \hat{\varrho}^{1}+\operatorname{div}\left(\hat{\varrho}^{1} \hat{v}^{0}\right)=-\operatorname{div}\left(\hat{\varrho}^{0} \hat{v}^{1}\right), \quad \hat{\varrho}^{1}(x, 0)=\varrho^{1}(x):=\varrho^{0}(x)-\varrho^{\mathrm{eq}}(x) .
$$

Since $\hat{\varrho}^{0} \in W_{p, \infty}^{1,1}\left(Q_{T}\right) \cap W_{p}^{2,0}\left(Q_{T}\right)$ by construction, the estimate of Lemma 20 applies (with the choices $\sigma=\hat{\varrho}^{1}, v=\hat{v}^{0}$ and $w:=\hat{v}^{1}$ ). Hence, invoking also (102),

$$
\begin{aligned}
\left\|\hat{\varrho}^{1}\right\|_{W_{p, \infty}^{1,1}\left(Q_{T}\right)}^{p} \leq & C \exp \left(c \int_{0}^{T}\left[\left\|\hat{v}_{x}^{0}(\tau)\right\|_{L^{\infty}(\Omega)}+\left\|\hat{v}_{x, x}^{0}(\tau)\right\|_{L^{p}(\Omega)}+1\right] d \tau\right) \times \\
& \times\left[\left\|\varrho^{1}\right\|_{W^{1, p}(\Omega)}^{p}+\left\|\hat{\varrho}^{0}\right\|_{W_{p}^{2,0}\left(Q_{T}\right)}^{p}\left\|\hat{v}^{1}\right\|_{L^{\infty}\left(Q_{T}\right)}^{p}\right. \\
& \left.+\left\|\hat{\varrho}^{0}\right\|_{W_{p, \infty}^{1,1}\left(Q_{T}\right)}^{p}\left\|\hat{v}^{1}\right\|_{W_{p}^{2,0}\left(Q_{T}\right)}^{p}\right] \\
\leq & C_{T}\left(\left\|\varrho^{1}\right\|_{W^{1, p}(\Omega)}^{p}+\left\|\hat{v}^{1}\right\|_{W_{p}^{2,1}\left(Q_{T}\right)}^{p}\right) \leq C\left(R_{0}, T\right) R_{1}^{p} .
\end{aligned}
$$

The latter and (102) now entail

$$
\left\|\hat{q}^{0}-q^{\mathrm{eq}}\right\|_{W_{p}^{2,1}\left(Q_{T}\right)}+\left\|\hat{v}^{0}-v^{\mathrm{eq}}\right\|_{W_{p}^{2,1}\left(Q_{T}\right)}+\left\|\hat{\varrho}^{0}-\varrho^{\mathrm{eq}}\right\|_{W_{p, \infty}^{1,1}\left(Q_{T}\right)} \leq C R_{1} .
$$

Thus, it also follows that $\left\|\hat{\varrho}^{0}-\varrho^{\mathrm{eq}}\right\|_{L^{\infty}\left(Q_{T}\right)} \leq C R_{1}$. Therefore

$$
\varrho_{\max }-\hat{\varrho}^{0}(x, t) \geq \varrho_{\max }-\varrho^{\mathrm{eq}}(x)-C R_{1}, \quad \hat{\varrho}^{0}(x, t)-\varrho_{\min } \geq \varrho^{\mathrm{eq}}(x)-\varrho_{\min }-C R_{1} .
$$


By definition, the equilibrium density remains in the thresholds, that is, $M\left(\varrho^{\mathrm{eq}}, 0\right)<$ $+\infty$ (see (69)). If $R_{1}$ is small enough, for instance if it satisfies the condition

$$
R_{1} \leq \frac{1}{2 C} \min _{x \in \bar{\Omega}}\left\{\varrho_{\max }-\varrho^{\mathrm{eq}}(x), \varrho^{\mathrm{eq}}(x)-\varrho_{\min }\right\},
$$

we can show that

$$
M\left(\hat{\varrho}^{0}, T\right)=\operatorname{esssup}_{Q_{T}} \max \left\{\frac{1}{\varrho_{\max }-\hat{\varrho}^{0}}, \frac{1}{\hat{\varrho}^{0}-\varrho_{\min }}\right\} \leq 2 M\left(\varrho^{\mathrm{eq}}, 0\right)<+\infty .
$$

We define $\zeta^{\mathrm{eq}}(x):=\eta^{N-1} \cdot \nabla_{\rho} k\left(\rho^{\mathrm{eq}}(x)\right)$. Multiplying (16) with $\bar{V}$, we see that $\zeta^{\text {eq }}$ satisfies

$$
\operatorname{div}\left(v^{\mathrm{eq}}-d\left(\varrho^{\mathrm{eq}}, q^{\mathrm{eq}}\right)\left(\nabla \zeta^{\mathrm{eq}}-\hat{b}(x)\right)+A\left(\varrho^{\mathrm{eq}}, q^{\mathrm{eq}}\right)\left(\nabla q^{\mathrm{eq}}-\tilde{b}(x)\right)\right)=0 .
$$

Since $\hat{\zeta}^{0}$ is constructed solving (51), the difference $y:=\hat{\zeta}^{0}-\zeta^{\mathrm{eq}}$ satisfies

$$
\begin{aligned}
-\operatorname{div}\left(d^{0} \nabla y\right)= & -\operatorname{div}\left(\hat{v}^{0}-v^{\mathrm{eq}}+\left(d^{0}-d^{\mathrm{eq}}\right)\left(\hat{b}(x)-\nabla \zeta^{\mathrm{eq}}\right)\right) \\
& -\operatorname{div}\left(A^{\mathrm{eq}} \cdot\left(\nabla q^{\mathrm{eq}}-\tilde{b}(x)\right)-A^{0}\left(\nabla \hat{q}^{0}-\tilde{b}(x)\right)\right)
\end{aligned}
$$

where zero superscript of a coefficient means evaluation at $\left(\hat{\varrho}^{0}, \hat{q}^{0}\right)$, while eq superscript means evaluation at $\left(\hat{\varrho}^{\mathrm{eq}}, \hat{q}^{\mathrm{eq}}\right)$. Thus, elementary calculations show that also

$$
\begin{aligned}
\left\|\hat{\zeta}^{0}-\zeta^{\mathrm{eq}}\right\|_{W_{p}^{2,0}\left(Q_{T}\right)} & \leq C\left(\left\|\hat{q}^{0}-q^{\mathrm{eq}}\right\|_{W_{p}^{2,1}\left(Q_{T}\right)}+\left\|\hat{v}^{0}-v^{\mathrm{eq}}\right\|_{W_{p}^{2,1}\left(Q_{T}\right)}+\left\|\hat{\varrho}^{0}-\varrho^{\mathrm{eq}}\right\|_{W_{p, \infty}^{1,1}\left(Q_{T}\right)}\right) \\
& \leq C R_{1} .
\end{aligned}
$$

The nonlinear map. Consider now $\left(r^{*}, w^{*}\right)$ given in ${ }_{0} \mathcal{Y}_{T}$. We define $q^{*}:=\hat{q}^{0}+r^{*}$ and $v^{*}=\hat{v}^{0}+w^{*}$. Following (59), we introduce $\varrho^{*}:=\mathscr{C}\left(v^{*}\right)$. Then, the difference $\sigma^{*}:=\varrho^{*}-\hat{\varrho}^{0}$ is a solution to

$$
\partial_{t} \sigma^{*}+\operatorname{div}\left(\sigma^{*} v^{*}\right)=-\operatorname{div}\left(\hat{\varrho}^{0} w^{*}\right), \quad \sigma^{*}(x, 0)=0 .
$$

Making use of Lemma $20\left(\sigma^{*}=\sigma\right.$ and $v^{*}=v, w^{*}=w$ therein), we get

$$
\begin{aligned}
\left\|\varrho^{*}-\hat{\varrho}^{0}\right\|_{W_{p, \infty}^{1,1}\left(Q_{T}\right)}^{p} \leq & C \exp \left(c \int_{0}^{T}\left[\left\|v_{x}^{*}(\tau)\right\|_{L^{\infty}(\Omega)}+\left\|v_{x, x}^{*}(\tau)\right\|_{L^{p}(\Omega)}+1\right] d \tau\right) \times \\
& \times\left(\left\|\hat{\varrho}^{0}\right\|_{W_{p}^{2,0}\left(Q_{T}\right)}^{p}\left\|w^{*}\right\|_{L^{\infty}\left(Q_{T}\right)}^{p}+\left\|\hat{\varrho}^{0}\right\|_{W_{p, \infty}^{1,1}\left(Q_{T}\right)}^{p}\left\|w^{*}\right\|_{W_{p}^{2,0}\left(Q_{T}\right)}^{p}\right) \\
\leq & \phi_{0}\left(T, R_{0},\left\|w^{*}\right\|_{W_{p}^{2,1}\left(Q_{T}\right)}\right)\left\|w^{*}\right\|_{W_{p}^{2,1}\left(Q_{T}\right)}^{p},
\end{aligned}
$$

with a certain continuous function $\phi_{0}$ increasing of its arguments. Hence, use of the continuous embedding $W_{p, \infty}^{1,1} \subset L^{\infty}$ yields $\left\|\varrho^{*}-\hat{\varrho}^{0}\right\|_{L^{\infty}\left(Q_{T}\right)} \leq \phi_{0}\left(T, R_{0}\right.$, $\left.\left\|w^{*}\right\|_{W_{p}^{2,1}\left(Q_{T}\right)}\right)\left\|w^{*}\right\|_{W_{p}^{2,1}\left(Q_{T}\right)}$. We recall (106) to show that, under the condition $\phi_{0}\left(T, R_{0}, \mathscr{V}\left(T ; w^{*}\right)\right) \mathscr{V}\left(T ; w^{*}\right) \leq \frac{1}{4} \min _{x \in \bar{\Omega}}\left\{\varrho_{\max }-\varrho^{\mathrm{eq}}(x), \varrho^{\mathrm{eq}}(x)-\varrho_{\min }\right\}=: a_{0}$, 
we can guarantee that $M\left(\varrho^{*}, T\right)<+\infty$ globally.

The vector $\hat{u}_{0}:=\left(\hat{q}^{0}, 1, \hat{\varrho}^{0}, \hat{v}^{0}\right)$ is in $\mathcal{X}_{T, I}$ under the condition (105). Given $\left(r^{*}, w^{*}\right) \in{ }_{0} \mathcal{Y}_{T}$ satisfying (108), we define $u^{*}:=\left(\hat{q}^{0}+r^{*}, 1, \mathscr{C}\left(\hat{v}^{0}+w^{*}\right), \hat{v}^{0}+w^{*}\right)$ (cp. (60)), and we see by the latter arguments that $u^{*} \in \mathcal{X}_{T, I}$ too. Thus, we can make sense of the operators $\left(g^{1}\right)^{\prime}\left(u^{*}, \hat{u}^{0}\right),\left(h^{1}\right)^{\prime}\left(u^{*}, \hat{u}^{0}\right)$ and $\left(f^{1}\right)^{\prime}\left(u^{*}, \hat{u}^{0}\right)$ in the right-hand of the equations (61), (62), (64) on the entire interval [0,T].

If we can solve the linear system (61), (62), (63), (64) for $(r, \chi, \zeta, w)$, we obtain a globally defined solution in ${ }_{0} \mathcal{X}_{T}$, and we can meaningfully define $\mathcal{T}^{1}\left(r^{*}, w^{*}\right):=$ $(r, w)$. We shall prove the solvability by linear continuation on the base of the continuity estimates, that we are in the position to prove next.

\subsection{Continuity estimates}

We need at first an estimate for the operators $\left(g^{1}\right)^{\prime},\left(h^{1}\right)^{\prime}$ and $\left(f^{1}\right)^{\prime}$. We shall prove it for general body forces $b=b(x, t)$, even if the statement of Theorem 3 requires only $b=b(x)$.

Lemma 21. Assume that the initial data satisfy (105). Consider $\hat{u}^{0}:=\left(\hat{q}^{0}, 1, \hat{\varrho}^{0}, \hat{v}^{0}\right)$ $\in \mathcal{X}_{T, I}$ with $\hat{\varrho}^{0} \in W_{p}^{2,0}\left(Q_{T}\right)$ constructed in Sect. 10.1. For a given $\left(r^{*}, w^{*}\right) \in{ }_{0} \mathcal{Y}_{T}$ satisfying (108), we define $u^{*}:=\left(\hat{q}^{0}+r^{*}, 1, \mathscr{C}\left(\hat{v}^{0}+w^{*}\right), \hat{v}^{0}+w^{*}\right) \in \mathcal{X}_{T, I}$ (cf. (60), Sect. $r m$ 10.1). We further consider $(r, w) \in 0_{0} \mathcal{Y}_{T}$, and we denote by $\sigma$ the function obtained via solution of (63). We define $\bar{u}:=(r, 1, \sigma, w) \in{ }_{0} \mathcal{X}_{T}$. Then the operators $\left(g^{1}\right)^{\prime},\left(h^{1}\right)^{\prime}$ and $\left(f^{1}\right)^{\prime}$ on the right-hand side of $(61),(62),(63)$ satisfy

$$
\begin{aligned}
& \left\|\left(g^{1}\right)^{\prime}\left(u^{*}, \hat{u}^{0}\right) \bar{u}\right\|_{L^{p}\left(Q_{t}\right)}^{p}+\left\|\left(h^{1}\right)^{\prime}\left(u^{*}, \hat{u}^{0}\right) \bar{u}\right\|_{W_{p}^{1,0}\left(Q_{t}\right)}^{p}+\left\|\left(f^{1}\right)^{\prime}\left(u^{*}, \hat{u}^{0}\right) \bar{u}\right\|_{L^{p}\left(Q_{t}\right)}^{p} \\
& \quad \leq K_{2}^{*}(t) \int_{0}^{t} \mathscr{V}^{p}(s) K_{1}^{*}(s) d s
\end{aligned}
$$

with functions $K_{1}^{*} \in L^{1}(0, T)$ and $K_{2}^{*} \in L^{\infty}(0, T)$. There is a continuous function $\Phi^{*}\left(t, a_{1}, a_{2}\right)$ defined for all $t, a_{1}, a_{2} \geq 0$, such that

$$
\left\|K_{1}^{*}\right\|_{L^{1}(0, t)},\left\|K_{2}^{*}\right\|_{L^{\infty}(0, t)} \leq \Phi^{*}\left(t, \mathscr{V}^{*}(t), R_{0}\right)
$$

for all $t \leq T$, where $\mathscr{V}(t):=\mathscr{V}(t ; r)+\mathscr{V}(t ; w), \mathscr{V}^{*}(t):=\mathscr{V}\left(t ; r^{*}\right)+\mathscr{V}\left(t ; w^{*}\right)$ and $R_{0}$ is defined in (100).

Proof. The estimates of $\left(g^{1}\right)^{\prime},\left(f^{1}\right)^{\prime}$ were performed in [5] for the corresponding norms. They can be translated one to one to the present context. In adapting the proof, recall also that the numbers $M\left(\varrho^{*}, T\right)$ and $M\left(\hat{\varrho}^{0}, T\right)$ are finite by construction. We consider here the factor $\left(h^{1}\right)^{\prime}$, which is treated with similar arguments. We recall that

$$
\begin{aligned}
h^{1} & =h^{1}(x, t, q, \varrho) \\
& =d(\varrho, q)\left(\hat{b}(x, t)-\nabla \hat{\zeta}^{0}(x, t)\right)+A(\varrho, q)\left(\tilde{b}(x, t)-\nabla \hat{q}^{0}(x, t)\right),
\end{aligned}
$$


where $\hat{\zeta}^{0}$ is constructed solving (51). The derivatives of $h^{1}$ are given by the following expressions:

$$
h_{q}^{1}=d_{q}\left(\hat{b}-\nabla \hat{\zeta}^{0}\right)+A_{q}\left(\tilde{b}-\nabla \hat{q}^{0}\right), \quad h_{\varrho}^{1}=d_{\varrho}\left(\hat{b}-\nabla \hat{\zeta}^{0}\right)+A_{\varrho}\left(\tilde{b}-\nabla \hat{q}^{0}\right),
$$

while the gradients in $x$ obey

$$
\begin{aligned}
\nabla h_{q}^{1}= & \left(d_{q, q} \nabla q+d_{q, \varrho} \nabla \varrho\right)\left(\hat{b}-\nabla \hat{\zeta}^{0}\right) \\
& +\left(A_{q, q} \nabla q+A_{q, \varrho} \nabla \varrho\right)\left(\tilde{b}-\nabla \hat{q}^{0}\right)+d_{q}\left(\nabla \hat{b}-D^{2} \hat{\zeta}^{0}\right)+A_{q}\left(\nabla \tilde{b}-D^{2} \hat{q}^{0}\right), \\
\nabla h_{\varrho}^{1}= & \left(d_{\varrho, q} \nabla q+d_{\varrho, \varrho} \nabla \varrho\right)\left(\hat{b}-\nabla \hat{\zeta}^{0}\right) \\
& +\left(A_{\varrho, q} \nabla q+A_{\varrho, \varrho} \nabla \varrho\right)\left(\tilde{b}-\nabla \hat{q}^{0}\right)+d_{\varrho}\left(\nabla \hat{b}-D^{2} \hat{\zeta}^{0}\right)+A_{\varrho}\left(\nabla \tilde{b}-D^{2} \hat{q}^{0}\right) .
\end{aligned}
$$

Denote by $c_{1}^{*}$ a generic function depending on $M(\varrho, T)$ and $\|q\|_{L^{\infty}\left(Q_{T}\right)}$. Then the following estimates are obviously valid:

$$
\begin{aligned}
&\left|h_{q}^{1}\right|+\left|h_{\varrho}^{1}\right| \leq c_{1}^{*}\left(|\hat{b}|+|\tilde{b}|+\left|\nabla \hat{\zeta}^{0}\right|+\left|\nabla \hat{q}^{0}\right|\right) \\
&\left|\nabla h_{q}^{1}\right|+\left|\nabla h_{\varrho}^{1}\right| \leq c_{1}^{*}(|\nabla q|+|\nabla \varrho|)\left(|\hat{b}|+|\tilde{b}|+\left|\nabla \hat{\zeta}^{0}\right|+\left|\nabla \hat{q}^{0}\right|\right) \\
& \quad+c_{1}^{*}\left(\left|\hat{b}_{x}\right|+\left|\tilde{b}_{x}\right|+\left|D_{x, x}^{2} \hat{\zeta}^{0}\right|+\left|D_{x, x}^{2} \hat{q}^{0}\right|\right) .
\end{aligned}
$$

Using that $W^{1, p}(\Omega) \subset L^{\infty}(\Omega)$, it follows that

$$
\begin{aligned}
& \left\|h_{q}^{1}\right\|_{L^{\infty, p}\left(Q_{t}\right)}+\left\|h_{\varrho}^{1}\right\|_{L^{\infty, p}\left(Q_{t}\right)} \leq c_{1}^{*}\left(\|\hat{b}\|_{W_{p}^{1,0}\left(Q_{t}\right)}\right. \\
& \left.\quad+\|\tilde{b}\|_{W_{p}^{1,0}\left(Q_{t}\right)}+\left\|\hat{\zeta}^{0}\right\|_{W_{p}^{2,0}\left(Q_{t}\right)}+\left\|\hat{q}^{0}\right\|_{W_{p}^{2,0}\left(Q_{t}\right)}\right), \\
& \left\|\nabla h_{q}^{1}\right\|_{L^{p}\left(Q_{t}\right)}+\left\|\nabla h_{\varrho}^{1}\right\|_{L^{p}\left(Q_{t}\right)} \leq c_{1}^{*}\left(\|\nabla q\|_{L^{p, \infty}\left(Q_{t}\right)}+\|\nabla \varrho\|_{L^{p, \infty}\left(Q_{t}\right)}+1\right) \times \\
& \quad \times\left(\|\hat{b}\|_{W_{p}^{1,0}\left(Q_{t}\right)}+\|\tilde{b}\|_{W_{p}^{1,0}\left(Q_{t}\right)}+\left\|\hat{\zeta}^{0}\right\|_{W_{p}^{2,0}\left(Q_{t}\right)}+\left\|\hat{q}^{0}\right\|_{W_{p}^{2,0}\left(Q_{t}\right)}\right) .
\end{aligned}
$$

Next we turn to estimate $\left(h^{1}\right)^{\prime}\left(u^{*}, \hat{u}^{0}\right) \bar{u}$ in $W_{p}^{1,0}\left(Q_{t}\right)$. At first we notice that $\left(h^{1}\right)^{\prime}\left(u^{*}, \hat{u}^{0}\right) \bar{u}=h_{q}^{1}\left(u^{*}, \hat{u}^{0}\right) r+h_{\varrho}^{1}\left(u^{*}, \hat{u}^{0}\right) \sigma$. Thus

$$
\begin{aligned}
\left\|\left(h^{1}\right)^{\prime}\left(u^{*}, \hat{u}^{0}\right) \bar{u}\right\|_{L^{p}\left(Q_{t}\right)}^{p} & \leq \int_{0}^{t}\left(\left|h_{q}^{1}\left(u^{*}, \hat{u}^{0}\right)\right|_{L^{\infty}}^{p}+\left|h_{\varrho}^{1}\left(u^{*}, \hat{u}^{0}\right)\right|_{L^{\infty}}^{p}\right)\left(|r|_{L^{p}}^{p}+|\sigma|_{L^{p}}^{p}\right) d \tau \\
& \leq c_{1}^{*} \int_{0}^{t} K_{1}^{*}(\tau)\left(|r|_{L^{p}}^{p}+|\sigma|_{L^{p}}^{p}\right) d \tau
\end{aligned}
$$

with $c_{1}^{*}=c_{1}\left(M\left(\varrho^{*}, T\right), M\left(\hat{\varrho}^{0}, T\right),\left\|q^{*}\right\|_{L^{\infty}\left(Q_{T}\right)},\left\|\hat{q}^{0}\right\|_{L^{\infty}\left(Q_{T}\right)}\right)$, and

$$
K_{1}^{*}(\tau):=\|\hat{b}(\tau)\|_{W^{1, p}(\Omega)}+\|\tilde{b}(\tau)\|_{W^{1, p}(\Omega)}+\left\|\hat{\zeta}^{0}(\tau)\right\|_{W^{2, p}(\Omega)}+\left\|\hat{q}^{0}(\tau)\right\|_{W^{2, p}(\Omega)} .
$$

The function $K_{1}^{*}$ is integrable on $(0, t)$ with norm bounded by a function $\Phi_{t}^{*}$ of the required structure. Estimating $\left\|q^{*}\right\|_{L^{\infty}\left(Q_{t}\right)} \leq\left\|\hat{q}^{0}\right\|_{L^{\infty}\left(Q_{t}\right)}+C t^{\gamma} \mathscr{V}\left(r^{*} ; t\right)$, we see 
that

$$
\begin{aligned}
& \left\|\left(h^{1}\right)^{\prime}\left(u^{*}, \hat{u}^{0}\right) \bar{u}\right\|_{L^{p}\left(Q_{t}\right)}^{p} \\
& \quad \leq \phi\left(t, \mathscr{V}^{*}(t), R_{0}\right) \int_{0}^{t} K_{1}^{*}(\tau)\left(\|r(\tau)\|_{L^{p}(\Omega)}^{p}+\|\sigma(\tau)\|_{L^{p}(\Omega)}^{p}\right) d \tau \\
& \quad \leq \phi\left(t, \mathscr{V}^{*}(t), R_{0}\right) \int_{0}^{t} K_{1}^{*}(\tau)\left(\|r(\tau)\|_{L^{p}(\Omega)}^{p}+c_{0}\left\|\sigma_{x}(\tau)\right\|_{L^{p}(\Omega)}^{p}\right) d \tau .
\end{aligned}
$$

For the terms containing $\sigma_{x}$, we use the result of Lemma 20. It yields for $\tau \leq t$ that, in particular,

$$
\|\sigma(\tau)\|_{W^{1, p}(\Omega)} \leq K_{3}(\tau)\|w\|_{L^{\infty}\left(Q_{\tau}\right)}^{p}+K_{4}(\tau)\|w\|_{W_{p}^{2,0}\left(Q_{\tau}\right)}^{p}
$$

with

$$
\begin{aligned}
& K_{3}(\tau):=C e^{c \int_{0}^{\tau}\left[\left\|v_{x}^{*}\right\|_{L^{\infty}(\Omega)}+\left\|v_{x, x}^{*}\right\|_{L}{ }_{(\Omega)}+1\right] d s}\left\|\hat{\varrho}^{0}\right\|_{W_{p}^{2,0}\left(Q_{\tau}\right)}^{p}, \\
& K_{4}(\tau):=C e^{c \int_{0}^{\tau}\left[\left\|v_{x}^{*}\right\|_{L^{\infty}(\Omega)}+\left\|v_{x, x}^{*}\right\|_{L^{p}(\Omega)}+1\right] d s}\left\|\hat{\varrho}^{0}\right\|_{W_{p, \infty}^{1,1}\left(Q_{\tau}\right)}^{p} .
\end{aligned}
$$

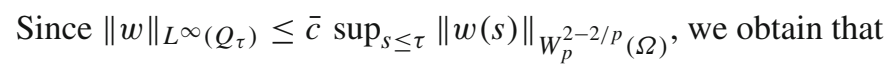

$$
\begin{aligned}
& \int_{0}^{t} K_{1}^{*}(\tau)\left\|\sigma_{x}(\tau)\right\|_{L^{p}(\Omega)}^{p} d \tau \\
& \quad \leq \max \left\{K_{3}(t), K_{4}(t)\right\} \int_{0}^{t} K_{1}^{*}(\tau)\left[\|w\|_{L^{\infty}\left(Q_{\tau}\right)}^{p}+\|w\|_{W_{p}^{2,0}\left(Q_{\tau}\right)}^{p}\right] d \tau \\
& \quad \leq \max \left\{K_{3}(t), K_{4}(t)\right\}(1+\bar{c})^{p} \int_{0}^{t} K_{1}^{*}(\tau) \mathscr{V}^{p}(w ; \tau) d \tau .
\end{aligned}
$$

Thus for $K_{2}^{*}(t):=C \phi\left(t, \mathscr{V}^{*}(t), R_{0}\right) \max \left\{K_{3}(t), K_{4}(t), 1\right\}$, it follows that

$$
\begin{aligned}
& \left\|\left(h^{1}\right)^{\prime}\left(u^{*}, \hat{u}^{0}\right) \bar{u}\right\|_{L^{p}\left(Q_{t}\right)}^{p} \leq \phi\left(t, \mathscr{V}^{*}(t), R_{0}\right) \int_{0}^{t} K_{1}^{*}(\tau)\|r(\tau)\|_{L^{p}(\Omega)}^{p} d \tau \\
& +\phi\left(t, \mathscr{V}^{*}(t), R_{0}\right) c_{0} \max \left\{K_{3}(t), K_{4}(t)\right\}(1+\bar{c})^{p} \int_{0}^{t} K_{1}^{*}(\tau) \mathscr{V}^{p}(w ; \tau) d \tau \\
& \left\|\left(h^{1}\right)^{\prime}\left(u^{*}, \hat{u}^{0}\right) \bar{u}\right\|_{L^{p}\left(Q_{t}\right)}^{p} \leq K_{2}^{*}(t) \int_{0}^{t} K_{1}^{*}(\tau) \mathscr{V}^{p}(\tau) d \tau
\end{aligned}
$$

We can prove a similar estimate for $\left\|\nabla\left(\left(h^{1}\right)^{\prime}\left(u^{*}, \hat{u}^{0}\right) \bar{u}\right)\right\|_{L^{p}\left(Q_{t}\right)}^{p}$. First we notice that

$$
\begin{aligned}
\nabla\left(\left(h^{1}\right)^{\prime}\left(u^{*}, \hat{u}^{0}\right) \bar{u}\right)= & \nabla\left(h_{q}^{1}\left(u^{*}, \hat{u}^{0}\right) r+h_{\varrho}^{1}\left(u^{*}, \hat{u}^{0}\right) \sigma\right) \\
= & \nabla h_{q}^{1}\left(u^{*}, \hat{u}^{0}\right) r+\nabla h_{\varrho}^{1}\left(u^{*}, \hat{u}^{0}\right) \sigma+h_{q}^{1}\left(u^{*}, \hat{u}^{0}\right) \nabla r \\
& \quad+h_{\varrho}^{1}\left(u^{*}, \hat{u}^{0}\right) \nabla \sigma .
\end{aligned}
$$

As before (see (111)), $\left\|h_{q}^{1} \nabla r+h_{\varrho}^{1} \nabla \sigma\right\|_{L^{p}\left(Q_{t}\right)}^{p} \leq c_{1}^{*} \int_{0}^{t} K_{1}^{*}(\tau)\left(\left\|r_{x}\right\|_{L^{p}(\Omega)}^{p}+\right.$ $\left.\left\|\sigma_{x}\right\|_{L^{p}(\Omega)}^{p}\right) d \tau$. Treating $\sigma_{x}$ as in (112), we obtain that $\left\|h_{q}^{1} \nabla r+h_{\varrho}^{1} \nabla \sigma\right\|_{L^{p}\left(Q_{t}\right)}^{p} \leq$ $K_{2}^{*}(t) \int_{0}^{t} K_{1}^{*}(\tau) \mathscr{V}^{p}(\tau) d \tau$. 
On the other hand, (110) yields

$$
\begin{aligned}
\| & \nabla h_{q}^{1}\left(u^{*}, \hat{u}^{0}\right) r+\nabla h_{\varrho}^{1}\left(u^{*}, \hat{u}^{0}\right) \sigma \|_{L^{p}\left(Q_{t}\right)}^{p} \\
\leq & c_{1}^{*}\left(\left\|\nabla q^{*}\right\|_{L^{p, \infty}\left(Q_{t}\right)}+\left\|\nabla \hat{q}^{0}\right\|_{L^{p, \infty}\left(Q_{t}\right)}+\left\|\nabla \varrho^{*}\right\|_{L^{p, \infty}\left(Q_{t}\right)}+\left\|\nabla \hat{\varrho}^{0}\right\|_{L^{p, \infty}\left(Q_{t}\right)}+1\right)^{p} \times \\
\quad & \times \int_{0}^{t}\left(\|\hat{b}\|_{W^{1, p}(\Omega)}+\|\tilde{b}\|_{W^{1, p}(\Omega)}+\left\|\hat{\zeta}^{0}\right\|_{W^{2, p}(\Omega)}+\left\|\hat{q}^{0}\right\|_{W^{2, p}(\Omega)}\right) \\
\quad & \times\left(\|r\|_{L^{\infty}(\Omega)}^{p}+\|\sigma\|_{L^{\infty}(\Omega)}^{p}\right) d \tau .
\end{aligned}
$$

This implies that

$$
\left\|\nabla h_{q}^{1} r+\nabla h_{\varrho}^{1} \sigma\right\|_{L^{p}\left(Q_{t}\right)}^{p} \leq \tilde{K}_{2}^{*}(t) \int_{0}^{t} \tilde{K}_{1}^{*}(\tau)\left(\|r(\tau)\|_{L^{\infty}(\Omega)}^{p}+c_{0}\left\|\sigma_{x}(\tau)\right\|_{L^{p}(\Omega)}^{p}\right) d \tau .
$$

Again, we treat $\sigma_{x}$ by means of (112). The claim follows.

Next we prove the main continuity estimate. We apply Proposition 11 to (61), (62). Making use of the fact that $r(0, x)=0$ in $\Omega$, we get the estimate

$$
\begin{aligned}
& \mathscr{V}(t ; r)+\|\chi\|_{W_{p}^{2,0}\left(Q_{t}\right)} \\
& \leq C \tilde{\Psi}_{1, T}\left(\left\|g^{1}\right\|_{L^{p}\left(Q_{t}\right)}+\left\|\left(h^{1}\right)^{\prime}\left(u^{*}, \hat{u}^{0}\right) \bar{u}\right\|_{W_{p}^{1,0}\left(Q_{t}\right)}+\|w\|_{W_{p}^{1,0}\left(Q_{t}\right)}\right) \\
& \leq C \tilde{\Psi}_{1, T}\left(\left\|\hat{g}^{0}\right\|_{L^{p}\left(Q_{t}\right)}+\|w\|_{W_{p}^{1,0}\left(Q_{t}\right)}\right) \\
& \quad+C \tilde{\Psi}_{1, T}\left(\left\|\left(g^{1}\right)^{\prime}\left(u^{*}, \hat{u}^{0}\right) \bar{u}\right\|_{L^{p}\left(Q_{t}\right)}+\left\|\left(h^{1}\right)^{\prime}\left(u^{*}, \hat{u}^{0}\right) \bar{u}\right\|_{W_{p}^{1,0}\left(Q_{t}\right)}\right) .
\end{aligned}
$$

Here $\tilde{\Psi}_{1, T}=\max \left\{\Psi_{1, T}, \Phi_{T}\right\}$ depends continuously on the data. We then apply Proposition 12 to (64) and obtain

$$
\begin{aligned}
\mathscr{V}(T ; w) & \leq C \tilde{\Psi}_{2, T}\left(\left\|f^{1}\right\|_{L^{p}\left(Q_{t}\right)}+\|\nabla \chi\|_{L^{p}\left(Q_{t}\right)}\right) \\
& \leq C \tilde{\Psi}_{2, T}\left(\left\|\hat{f}^{0}\right\|_{L^{p}\left(Q_{t}\right)}+\|\nabla \chi\|_{L^{p}\left(Q_{t}\right)}+\left\|\left(f^{1}\right)^{\prime}\left(u^{*}, \hat{u}^{0}\right) \bar{u}\right\|_{L^{p}\left(Q_{t}\right)}\right),
\end{aligned}
$$

again with some $\tilde{\Psi}_{2, T}$ depending on $T$ and $\left.\sup _{s \leq t}\left[\varrho^{*}(s)\right]_{C^{\alpha}(\Omega)}\right)$. We estimate $\|\nabla \chi\|_{L^{p}\left(Q_{t}\right)}$ by means of (113). We next raise both (113) and (114) to the $p^{\text {th }}$ power, add both inequalities, and get for the function $\mathscr{V}(t):=\mathscr{V}(t ; r)+\mathscr{V}(t ; w)+\|\chi\|_{W_{p}^{2,0}\left(Q_{t}\right)}$ the inequality

$$
\begin{aligned}
\mathscr{V}^{p}(t) \leq & C\left(\tilde{\Psi}_{1, T}^{p}+\tilde{\Psi}_{2, T}^{p}\right)\left(\left\|\hat{g}^{0}\right\|_{L^{p}\left(Q_{T}\right)}^{p}+\left\|\hat{f}^{0}\right\|_{L^{p}\left(Q_{T}\right)}^{p}+\|w\|_{W_{p}^{1,0}\left(Q_{t}\right)}^{p}\right. \\
& +\left\|\left(g^{1}\right)^{\prime}\left(u^{*}, \hat{u}^{0}\right) \bar{u}\right\|_{L^{p}\left(Q_{t}\right)}^{p}+\left\|\left(f^{1}\right)^{\prime}\left(u^{*}, \hat{u}^{0}\right) \bar{u}\right\|_{L^{p}\left(Q_{t}\right)}^{p} \\
& \left.+\left\|\left(h^{1}\right)^{\prime}\left(u^{*}, \hat{u}^{0}\right) \bar{u}\right\|_{W_{p}^{1,0}\left(Q_{t}\right)}^{p}\right) .
\end{aligned}
$$

Then we make use of $\|w\|_{W_{p}^{1,0}\left(Q_{t}\right)}^{p} \leq \int_{0}^{t} \mathscr{V}^{p}(s) d s$, and we apply the Lemma 21 to find that

$$
\mathscr{V}^{p}(t) \leq C\left(\tilde{\Psi}_{1, T}^{p}+\tilde{\Psi}_{2, T}^{p}\right)\left(\left\|\hat{g}^{0}\right\|_{L^{p}\left(Q_{T}\right)}^{p}+\left\|\hat{f}^{0}\right\|_{L^{p}\left(Q_{T}\right)}^{p}+K_{2}^{*}(t) \int_{0}^{t} K_{1}^{*}(s) \mathscr{V}^{p}(s) d s\right) .
$$


The Gronwall inequality implies that

$$
\begin{aligned}
\mathscr{V}^{p}(t) \leq & C\left(\tilde{\Psi}_{1, T}^{p}+\tilde{\Psi}_{2, T}^{p}\right) \exp \left[C\left(\tilde{\Psi}_{1, T}^{p}+\tilde{\Psi}_{2, T}^{p}\right) K_{2}^{*}(t) \int_{0}^{t} K_{1}^{*}(s) d s\right] \\
& \times\left(\left\|\hat{g}^{0}\right\|_{L^{p}\left(Q_{T}\right)}^{p}+\left\|\hat{f}^{0}\right\|_{L^{p}\left(Q_{T}\right)}^{p}\right) .
\end{aligned}
$$

We thus have proved the following continuity estimate.

Proposition 22. We define $R_{0}$ via (100). Suppose that $\left(r^{*}, w^{*}\right) \in{ }_{0} \mathcal{Y}_{T}$ satisfy the condition (108). Then $(r, w)=\mathcal{T}^{1}\left(r^{*}, w^{*}\right)$ is well defined in ${ }_{0} \mathcal{Y}_{T}$. Moreover, there is a continuous function $\Psi_{7}=\Psi_{7}\left(T, R_{0}, \eta\right)$, increasing of all arguments and finite for all $\phi_{0}\left(T, R_{0}, \eta\right) \eta<\frac{1}{4 M\left(\varrho^{e q}, 0\right)}$ such that

$$
\mathscr{V}(T) \leq \Psi_{7}\left(T, R_{0}, \mathscr{V}^{*}(T)\right)\left(\left\|\hat{g}^{0}\right\|_{L^{p}\left(Q_{T}\right)}+\left\|\hat{f}^{0}\right\|_{L^{p}\left(Q_{T}\right)}\right)
$$

10.3. Existence of a unique fixed-point of $\mathcal{T}^{1}$

We are now in the position to prove a self-mapping property for sufficiently small data. We recall the definitions (99), (100) of the critical norms $R_{0}, R_{1}$. We denote $u^{\mathrm{eq}}=\left(q^{\mathrm{eq}}, \zeta^{\mathrm{eq}}, \varrho^{\mathrm{eq}}, v^{\mathrm{eq}}\right)$ and let $\hat{u}^{0}:=\left(\hat{q}^{0}, \hat{\zeta}^{0}, \hat{\varrho}^{0}, \hat{v}^{0}\right)$ and $\hat{u}^{1}:=u^{\mathrm{eq}}-\hat{u}^{0}$. In (104), (107), we just proved that $\left\|\hat{u}^{1}\right\|_{\mathcal{X}_{T}} \leq C R_{1}$. Recalling that the operator $\tilde{\mathscr{A}}$ is continuously differentiable into the space $\mathcal{Z}_{T}$ defined in (38), and that $\widetilde{A}\left(u^{\mathrm{eq}}\right)=0$ by the definition of an equilibrium solution, we can verify that

$$
\tilde{\mathscr{A}}\left(\hat{u}^{0}\right)=\tilde{\mathscr{A}}\left(\hat{u}^{\mathrm{eq}}+\hat{u}^{1}\right)=\tilde{\mathscr{A}}\left(\hat{u}^{\mathrm{eq}}+\hat{u}^{1}\right)-\tilde{\mathscr{A}}\left(\hat{u}^{\mathrm{eq}}\right)=\int_{0}^{1} \tilde{\mathscr{A}}^{\prime}\left(\hat{u}^{\mathrm{eq}}+\theta \hat{u}^{1}\right) d \theta \hat{u}^{1} .
$$

Thus $\left\|\tilde{\mathscr{A}}\left(\hat{u}^{0}\right)\right\|_{Z_{T}} \leq C R_{1}$. The definitions of $\hat{g}^{0}$ and $\hat{f}^{0}$ in (58) show that

$$
\left\|\hat{g}^{0}\right\|_{L^{p}\left(Q_{T}\right)}+\left\|\hat{f}^{0}\right\|_{L^{p}\left(Q_{T}\right)}=\left\|\widetilde{\mathscr{A}}^{1}\left(\hat{u}^{0}\right)\right\|_{L^{p}\left(Q_{T}\right)}+\left\|\tilde{\mathscr{A}}^{4}\left(\hat{u}^{0}\right)\right\|_{L^{p}\left(Q_{T}\right)} \leq \bar{C} R_{1} .
$$

These considerations allow to state and prove the main properties of $\mathcal{T}^{1}$.

Lemma 23. We define $R_{0}$ via (100) and $R_{1}$ via (99). For $\phi_{0}$ and $a_{0}$ defined in (108), we define $\eta_{0}>0$ as the smallest positive number such that $\phi_{0}\left(T, R_{0}, \eta_{0}\right) \eta_{0}=$ $a_{0}$. We define $\bar{R}_{1}=\min \left\{1 /\left(2 C M\left(\varrho^{e q}, 0\right)\right), \eta_{0} /\left(\bar{C} \Psi_{7}\left(T, R_{0}, \eta_{0}\right)\right)\right\}$ with $\Psi_{7}$ from Proposition 22, C from (105), and $\bar{C}$ from (116). If $R_{1} \leq \bar{R}_{1}$, the map $\mathcal{T}^{1}$ is well defined and possesses a unique fixed-point.

Proof. If $w^{*}$ satisfies (108) and if $R_{1}$ satisfies (105), $\mathcal{T}^{1}\left(r^{*}, w^{*}\right)$ is well defined in $\mathcal{Y}_{T}$. We apply Proposition 22, use (116) and obtain

$$
\left\|\mathcal{T}^{1}\left(r^{*}, w^{*}\right)\right\| \mathcal{Y}_{T} \leq \Psi_{7}\left(T, R_{0},\left\|\left(r^{*}, w^{*}\right)\right\| \mathcal{Y}_{T}\right)\left(\left\|\hat{g}^{0}\right\|_{L^{p}\left(Q_{T}\right)}+\left\|\hat{f}^{0}\right\|_{L^{p}\left(Q_{T}\right)}\right) \leq \eta_{0} .
$$

We consider the iteration $\bar{u}^{n+1}:=\mathcal{T}^{1}\left(\bar{u}^{n}\right)$, starting at zero. The sequence $\left(q^{n}, \zeta^{n}\right.$, $\left.\varrho^{n}, v^{n}\right)$ is then uniformly bounded in $\mathcal{X}_{T}$. We show the contraction property with 
respect to the same lower-order norm than in Lemma 18 . There are $k_{0}, p_{0}>0$ such that the quantities

$$
\begin{aligned}
& E^{n}(t):=p_{0} \int_{t}^{t+t_{1}}\left\{\left|\nabla\left(r^{n}-r^{n-1}\right)\right|^{2}+\left|\nabla\left(\chi^{n}-\chi^{n-1}\right)\right|^{2}+\left|\nabla\left(w^{n}-w^{n-1}\right)\right|^{2}\right\} d x d s \\
& \quad+k_{0} \sup _{\tau \in\left[t, t+t_{1}\right]}\left\{\left\|\left(r^{n}-r^{n-1}\right)(\tau)\right\|_{L^{2}(\Omega)}^{2}+\left\|\left(\sigma^{n}-\sigma^{n-1}\right)(\tau)\right\|_{L^{2}(\Omega)}^{2}\right. \\
& \left.\quad+\left\|\left(w^{n}-w^{n-1}\right)(\tau)\right\|_{L^{2}(\Omega)}^{2}\right\}
\end{aligned}
$$

satisfy $E^{n+1}(t) \leq \frac{1}{2} E^{n}(t)$ for some fixed $t_{1}>0$ and every $t \in\left[0, T-t_{1}\right]$.

Funding Open Access funding enabled and organized by Projekt DEAL.

Open Access. This article is licensed under a Creative Commons Attribution 4.0 International License, which permits use, sharing, adaptation, distribution and reproduction in any medium or format, as long as you give appropriate credit to the original author(s) and the source, provide a link to the Creative Commons licence, and indicate if changes were made. The images or other third party material in this article are included in the article's Creative Commons licence, unless indicated otherwise in a credit line to the material. If material is not included in the article's Creative Commons licence and your intended use is not permitted by statutory regulation or exceeds the permitted use, you will need to obtain permission directly from the copyright holder. To view a copy of this licence, visit http://creativecommons.org/licenses/ by $/ 4.0 /$.

Publisher's Note Springer Nature remains neutral with regard to jurisdictional claims in published maps and institutional affiliations.

\section{A. Properties of the free energy density}

In this section we prove the statements of Sect. 3 devoted to the convex conjugate of the free energy density: Lemmas 4, 5 and 6.

We assume that $k$ satisfies the assumptions of Lemma 4. Notice that requiring $k$ essentially smooth on $S_{1}$, while positive homogeneous, induces that $k$ is also essentially smooth on $S_{\bar{V}}$. To see this, we consider any sequence $\left\{r^{m}\right\} \subset S_{\bar{V}}$ such that $r^{m} \rightarrow \bar{r}$ for $m \rightarrow \infty$, and $\bar{r}$ belongs to the relative boundary of $S_{\bar{V}}$, which means that there is $i \in\{1, \ldots, N\}$ such that $\bar{r}_{i}=0$. Then we define $y^{m}:=\bar{r}_{m} / \sum_{i=1}^{N} r_{i}^{m}$ which belongs to $S_{1}$ for all $m$, and satisfies $y_{i}^{m} \rightarrow 0$ for $m \rightarrow \infty$. Since $k$ is positively homogeneous, we have $\nabla_{\rho} k\left(r^{m}\right)=\nabla_{\rho} k\left(y^{m}\right)$. Thus, by the assumptions of Lemma 4 , we see that $\left|\nabla_{\rho} k\left(r^{m}\right)\right| \rightarrow+\infty$, which is the essential smoothness on $S_{\bar{V}}$.

Consider now $\mu \in \mathbb{R}^{N}$ arbitrary. Then we claim first that there exists a unique $\bar{r} \in S_{\bar{V}}$ such that

$$
f(\mu)=\sup _{r \in S_{\bar{V}}}\{\mu \cdot r-k(r)\}=\mu \cdot \bar{r}-k(\bar{r}) .
$$

Since $S_{\bar{V}}$ is bounded, we first notice that $\sup _{r \in S_{\bar{V}}}\{\mu \cdot r-k(r)\}=\max _{r \in \overline{S_{\bar{V}}}}\{\mu \cdot r-k(r)\}$. Thus, there is $\bar{r} \in \overline{S_{\bar{V}}}$ such that $\sup _{r \in S_{\bar{V}}}\{\mu \cdot r-k(r)\}=\mu \cdot \bar{r}-k(\bar{r})$. We want to show 
that $\bar{r}$ is an interior point. Since $S_{\bar{V}}$ is a convex set, we can find for every $a \in S_{\bar{V}}$ a $h>0$ such that $\bar{r}+h(a-\bar{r}) \in S_{\bar{V}}$. Due to the choice of $\bar{r}$

$$
\mu \cdot(\bar{r}+h(a-\bar{r}))-k(\bar{r}+h(a-\bar{r})) \leq \mu \cdot \bar{r}-k(\bar{r}),
$$

which yields $k(\bar{r}+h(a-\bar{r}))-k(\bar{r}) \geq h \mu \cdot(a-\bar{r})$ and $\lim _{h \searrow 0} \frac{k(\bar{r}+h(a-\bar{r}))-k(\bar{r})}{h}>-\infty$. The latter however contradicts the fact that $k$ is essentially smooth on $S_{\bar{V}}$ (cf. [28], Lemma 26.2). Thus, $\bar{r} \in S_{\bar{V}}$ is an interior point.

The uniqueness of $\bar{r}$ follows from the strict convexity of $k$ on $S_{\bar{V}}$.

Since $k$ is differentiable, and since $r \mapsto \mu \cdot r-k(r)$ attains its maximum in $\bar{r}$, we must have $(\nabla k(\bar{r})-\mu) \cdot \xi=0$ for every tangential vector $\xi \in \mathbb{R}^{N}$ such that $\xi \cdot \bar{V}=0$. Thus, there is $p \in \mathbb{R}$ such that $\mu=\nabla_{\rho} k(\bar{r})+p \bar{V}$. Multiplying with $\bar{r}$, use of the homogeneity of degree one implies that $\bar{r} \cdot \nabla_{\rho} k(\bar{r})=k(\bar{r})$, hence

$$
\sup _{r \in S_{\bar{V}}}\{\mu \cdot r-k(r)\}=\mu \cdot \bar{r}-k(\bar{r})=p \bar{r} \cdot \bar{V}=p,
$$

showing that $p=f(\mu)$. Due to the structure $f(\mu)=\mu \cdot \bar{r}-k(\bar{r})=\max _{r \in S_{\bar{V}}}\{\mu$. $r-k(r)\}$, we easily show that $f$ is differentiable in $\mu$ with $\nabla_{\mu} f(\mu)=\bar{r}$. In order to show the differentiability of higher order, we can exploit the identities

$$
\mu-f(\mu) \bar{V}=\nabla_{\rho} k\left(\nabla_{\mu} f(\mu)\right), \quad \bar{V} \cdot \nabla_{\mu} f(\mu)=1 .
$$

For a system of orthonormal vectors $\xi^{1}, \ldots, \xi^{N-1}$ for $\{\bar{V}\}^{\perp}$, and $\xi^{N}:=\bar{V} /|\bar{V}|$, we then have

$$
\begin{aligned}
\mu \cdot \xi^{j} & =\xi^{j} \cdot \nabla_{\rho} k\left(\sum_{i=1}^{N-1} \xi^{i} \cdot \nabla_{\mu} f(\mu) \xi^{i}+\frac{\xi^{N}}{|\bar{V}|}\right) \text { for } j=1, \ldots, N-1, \\
\frac{1}{|\bar{V}|} & =\xi^{N} \cdot \nabla_{\mu} f(\mu) .
\end{aligned}
$$

The latter can be viewed as an algebraic system of the form $F(X)=\left(\mu \cdot \xi^{1}, \ldots, \mu\right.$. $\left.\xi^{N-1}, \frac{1}{|\bar{V}|}\right)$ for the unknowns $X:=\left(\xi^{1} \cdot \nabla_{\mu} f(\mu), \ldots, \xi^{N} \cdot \nabla_{\mu} f(\mu)\right) \in \mathbb{R}^{N}$. The Jacobian of this system obeys

$$
\frac{\partial F_{j}}{\partial X_{i}}= \begin{cases}D^{2} k \xi^{i} \cdot \xi^{j} & \text { for } i=1, \ldots, N-1, j=1, \ldots, N-1, \\ 0 & \text { for } i=N, j=1, \ldots, N-1, \\ \delta_{i, N} & \text { for } i=1, \ldots, N, j=N\end{cases}
$$

where $D^{2} k$ is evaluated at $\nabla_{\mu} f(\mu)$. We can easily verify that $\left\{D^{2} k\left(\nabla_{\mu} f(\mu)\right) \xi^{i}\right.$. $\left.\xi^{j}\right\}_{i, j=1, \ldots, N-1}$ is strictly positive definite: A vector of the form $\sum_{j=1}^{N-1} \xi^{j} a_{j}, a \neq 0$ can never be parallel to $\nabla_{\mu} f(\mu)$, since multiplying with $\bar{V}$ yields a contradiction. On the other hand, the properties of $k$ guarantee that the kernel of $D^{2} k\left(\nabla_{\mu} f(\mu)\right)$ is the one-dimensional span of $\nabla_{\mu} f(\mu)$. 
Thus, the equations $F(X(\mu))=\left(\mu \cdot \xi^{1}, \ldots, \mu \cdot \xi^{N-1}, \frac{1}{|\bar{V}|}\right)$ define implicitly a map $\mu \mapsto X(\mu)$ of class $C^{1}\left(\mathbb{R}^{N}\right)$. This clearly implies that $f \in C^{2}\left(\mathbb{R}^{N}\right)$, and we obtain the formula

$$
D_{\mu_{k}, \mu_{i}}^{2} f(\mu)=\sum_{j=1}^{N} \frac{\partial X_{j}}{\partial \mu_{k}} \xi_{i}^{j} .
$$

If $k \in C^{3}\left(\mathbb{R}_{+}^{N}\right)$, we then differentiate again to obtain that $f$ is $C^{3}\left(\mathbb{R}^{N}\right)$. This proves the claims of Lemma 4 . The claims of Lemma 5 and 6 are also readily established (use (118) and (117)).

\section{B. Auxiliary statements}

For the proof of the following Lemma, we need the variable transformation in Sect. 4.1.

Lemma 24. We adopt the assumptions of Theorem 1 for the tensor $M: \mathbb{R}_{+}^{N} \rightarrow$ $\mathbb{R}^{N \times N}$, and we assume that $k: \mathbb{R}_{+}^{N} \rightarrow \mathbb{R}$ is given by (7). We assume moreover that there is a continuous function $C=C(|\rho|)$, bounded on compact subsets of $\overline{\mathbb{R}}^{N} \backslash\{0\}$, such that $B_{i, j}(\rho):=M_{i, j}(\rho) / \rho_{j}$, with entries belonging to $C^{1}\left(\mathbb{R}_{+}^{N}\right)$, satisfies for all $\rho \in \mathbb{R}_{+}^{N}$ the conditions

$$
\left|B_{i, j}(\rho)\right|+\rho_{k}\left|B_{i, j, \rho_{k}}(\rho)\right| \leq C(|\varrho|) \text { for all } i, j, k \in\{1, \ldots, N\}
$$

For $\varrho \in I$ and $q \in \mathbb{R}^{N-2}$, we denote $M(\varrho, q):=M\left(\sum_{\ell=1}^{N-2} R_{\ell}(\varrho, q) \eta^{\ell}+\varrho \eta^{N}\right)$, and recall the definitions (22), (23), (24) of the objects $\tilde{M}(\varrho, q), A(\varrho, q), d(\varrho, q)$ and the definition (21) of the nonlinear part $P(\varrho, q)$ of the pressure. For $\varrho \in I$, we define $m(\varrho):=\min \left\{1-\varrho / \varrho_{\max }, \varrho / \varrho_{\min }-1\right\}$. Then the following statements are valid: For all $\varrho \in I$ and $q \in \mathbb{R}^{N-2}$

- $|d(\varrho, q)|+|A(\varrho, q)|+\left|d_{q}(\varrho, q)\right|+\left|A_{q}(\varrho, q)\right| \leq c_{1} m(\varrho)$;

- $\left|d_{\varrho}(\varrho, q)\right|+\left|A_{\varrho}(\varrho, q)\right| \leq c_{2}$;

- The function $P_{\varrho}$ is positive and $c_{3}(m(\varrho))^{-1} \leq P_{\varrho}(\varrho, q) \leq c_{4}(m(\varrho))^{-1}$ with $c_{3}>0$; Moreover $\left|P_{q}(\varrho, q)\right| \leq c_{5}$.

Proof. For $\rho \in S_{\bar{V}}$ we consider the vector $u_{j}=-\rho_{j}\left(\bar{V}_{j}-1 / \varrho_{\min }\right)$ for $j=1, \ldots, N$. By the definition of $\varrho_{\min }$, all components of $u$ are positive. Moreover $\sum_{j=1}^{N} u_{j}=$ $\varrho / \varrho_{\min }-1$ by the definition of $S_{\bar{V}}$. Since $M 1^{N}=0$, the identity $M(\rho) \bar{V}=M(\rho)(\bar{V}-$ $\left.1^{N} / \varrho_{\min }\right)=-B(\rho) u$ holds. By assumption $|B(\rho)| \leq C(|\rho|)$, and therefore

$$
|M(\rho) \bar{V}| \leq C(|\rho|)|u| \leq C_{0}\left(\frac{\varrho}{\varrho_{\min }}-1\right) .
$$

Analogously, considering next $u_{j}:=\rho_{j}\left(\bar{V}_{j}-1 / \varrho_{\max }\right)$, we obtain that $|M(\rho) \bar{V}| \leq$ $C_{1}\left(1-\varrho / \varrho_{\max }\right)$, and overall that $|M(\rho) \bar{V}| \leq C m(\varrho)$. 
We next investigate the derivatives. To do so, we recall two properties of the map $\mathscr{R}(\varrho, q)$ (Sect. 4.1, (20)). For $\ell=1, \ldots, N-2$ direct computations yield for $i=$ $1, \ldots, N$

$$
\begin{aligned}
& \partial_{q_{\ell}} \mathscr{R}_{i}(\varrho, q)=D^{2} f e^{i} \cdot \xi^{\ell}-\frac{D^{2} f e^{i} \cdot 1^{N} D^{2} f \xi^{\ell} \cdot 1^{N}}{D^{2} f 1^{N} \cdot 1^{N}} \text { for } \ell=1, \ldots, N-2, \\
& \partial_{\varrho} \mathscr{R}_{i}(\varrho, q)=\frac{D^{2} f e^{i} \cdot 1^{N}}{D^{2} f 1^{N} \cdot 1^{N}} .
\end{aligned}
$$

In these formula, we evaluate $D^{2} f$ at $\mu=\sum_{\ell=1}^{N-2} q_{\ell} \xi^{\ell}+\mathscr{M}(\varrho, q) 1^{N}$. In the Section 4 of [14], we prove that $D^{2} f e^{i} \cdot 1^{N} \leq C_{0} D^{2} f 1^{N} \cdot 1^{N}$ for $i=1, \ldots, N$ (Lemma 4.3 (e)). Moreover, $\left|D^{2} f e^{i} \cdot a\right| \leq c_{a} \rho_{i}$ for any vector $a$ (cf. Lemma 4.3 (a)). From these properties, we infer that

$$
\frac{1}{\rho_{i}}\left|\partial_{q} \mathscr{R}_{i}(\varrho, q)\right| \leq \frac{\left|D^{2} f e^{i}\right|}{\rho_{i}}\left(1+C_{0} \max _{\ell=1, \ldots, N-2}\left|\xi^{\ell}\right|\right) \leq c, \quad\left|\partial_{\varrho} \mathscr{R}_{i}(\varrho, q)\right| \leq c .
$$

We again express $M_{i, j}(\rho) \bar{V}_{j}=-B_{i, j}(\rho) \rho_{j}\left(\bar{V}_{j}-1 / \varrho_{\min }\right)$, hence

$$
\partial_{\rho_{k}} M_{i, j}(\rho) \bar{V}_{j}=-B_{i, j, \rho_{k}}(\rho) \rho_{j}\left(\bar{V}_{j}-\frac{1}{\varrho_{\min }}\right)-B_{i, k}\left(\bar{V}_{k}-\frac{1}{\varrho_{\min }}\right),
$$

and therefore, it follows for $\ell=1, \ldots, N-2$ that

$\partial_{q_{\ell}} M_{i, j}(\mathscr{R}(\varrho, q)) \bar{V}_{j}=\sum_{k=1}^{N}\left(-B_{i, j, \rho_{k}}(\rho) \rho_{j}\left(\bar{V}_{j}-\frac{1}{\varrho_{\min }}\right)-B_{i, k}\left(\bar{V}_{k}-\frac{1}{\varrho_{\min }}\right)\right) \mathscr{R}_{k, q_{\ell}}$.

Since $-B_{i, j, \rho_{k}}(\rho) \rho_{j}\left(\bar{V}_{j}-1 / \varrho_{\min }\right)=-B_{i, j, \rho_{k}} u_{j}$, and, by assumption, $\left|B_{i, j, \rho_{k}}\right| \leq$ $C(|\rho|) / \rho_{k}$, we invoke that $\left|\mathscr{R}_{k, q_{\ell}}\right| \leq C \rho_{k}$ to show that

$$
\left|B_{i, j, \rho_{k}}(\rho) u_{j} \mathscr{R}_{k, q_{\ell}}\right| \leq C_{0}|u| \leq C_{0}\left(\frac{\varrho}{\varrho_{\min }}-1\right) .
$$

Moreover, by the same means

$$
\left.\mid B_{i, k}\left(\bar{V}_{k}-\frac{1}{\varrho_{\min }}\right)\right) \mathscr{R}_{k, q_{\ell}}|\leq| B_{i, k} u_{k}\left(\mathscr{R}_{k, q_{\ell}} / \rho_{k}\right)\left|\leq C_{1}\right| u \mid \leq C_{1}\left(\frac{\varrho}{\varrho_{\min }}-1\right) .
$$

Arguing the same for the other choice of $u$, it follows that $\left|\partial_{q \ell} M_{i, j}(\mathscr{R}(\varrho, q)) \bar{V}_{j}\right| \leq$ $C m(\varrho)$. The other estimates claimed have been verified for this special case of the function $k$ in the Section 4 of [14].

Remark 25. In the case that the matrix $M$ results from inversion of the MaxwellStefan equations, we notice that the matrix $B$ of Lemma 24 is nothing else but the pseudo-inverse of the Maxwell-Stefan matrix. It is shown in the paper [4] that natural assumptions on the binary diffusivities are sufficient for proving that the entries of $B$ consist of regular functions of the state variables. In particular, they satisfy the assumptions of Lemma 24. 
The following statement is directly taken from our paper [5]. There we must only adapt the definition of the parameters $m^{*}$ and $M^{*}$ according to (69), (68) in order to account for different density thresholds in the incompressible model.

Proposition 26. Assume that $R_{q}, \tilde{M}: I \times \mathbb{R}^{N-2} \rightarrow \mathbb{R}^{(N-2) \times(N-2)}$ are maps of class $C^{1}$ into the set of symmetric, positive definite matrices. Consider given $q^{*} \in$ $W_{p}^{2,1}\left(Q_{T} ; \mathbb{R}^{N-2}\right)$ and $\varrho^{*} \in W_{p, \infty}^{1,1}\left(Q_{T}\right)(p>3)$ such that the values of $\varrho^{*}$ are strictly contained in $I$ in $\overline{Q_{T}}$. Let $g \in L^{p}\left(Q_{T} ; \mathbb{R}^{N-2}\right)$ and $q^{0} \in W^{2-2 / p}(\Omega)$ such that $v \cdot \nabla q^{0}(x)=0$ in the sense of traces on $\partial \Omega$. Then, there is a unique $q \in$ $W_{p}^{2,1}\left(Q_{T} ; \mathbb{R}^{N-2}\right)$ solution to the problem

$$
\begin{aligned}
& R_{q}\left(\varrho^{*}, q^{*}\right) \partial_{t} q-\operatorname{div}\left(\tilde{M}\left(\varrho^{*}, q^{*}\right) \nabla q\right)=g \text { in } Q_{T}, \quad v \cdot \nabla q=0 \text { on } S_{T}, \\
& q(x, 0)=q^{0}(x) \text { in } \Omega
\end{aligned}
$$

Moreover there is a constant $C$ independent on $T, q, \varrho^{*}$ and $q^{*}$ such that for all $t \leq T$ and $0<\beta \leq 1$ :

$$
\begin{aligned}
& \mathscr{V}(t ; q) \leq C \bar{\Psi}_{1, t}\left[\left(1+\left[\varrho^{*}\right]_{C^{\beta, \frac{\beta}{2}}\left(Q_{t}\right)}\right)^{\frac{2}{\beta}}\left\|q^{0}\right\|_{W_{p}^{2-\frac{2}{p}}(\Omega)}+\|g\|_{L^{p}\left(Q_{t}\right)}\right] \\
& \bar{\Psi}_{1, t}=\bar{\Psi}_{1}\left(t, M^{*}(t),\left\|q^{*}(0)\right\|_{C^{\beta}(\Omega)}, \mathscr{V}\left(t ; q^{*}\right),\left[\varrho^{*}\right]_{C^{\beta, \frac{\beta}{2}}\left(Q_{t}\right)},\left\|\nabla \varrho^{*}\right\|_{L^{p, \infty}\left(Q_{t}\right)}\right),
\end{aligned}
$$

with a continuous function $\bar{\Psi}_{1}$ defined for all $t \geq 0$ and all numbers $a_{1}, \ldots, a_{5} \geq 0$. The function $\bar{\Psi}_{1}$ is increasing in all arguments and moreover $\bar{\Psi}_{1}\left(0, a_{1}, \ldots, a_{5}\right)=$ $\bar{\Psi}_{1}^{0}\left(a_{1}, a_{2}, a_{3}\right)$ is a function independent on the two last arguments.

We also recall some estimates of Hölder norms. This is also proved in [5].

Lemma 27. For $0 \leq \beta<\min \left\{1,2-\frac{5}{p}\right\}$ we define

$$
\gamma:= \begin{cases}\frac{1}{2}\left(2-\frac{5}{p}-\beta\right) & \text { for } 3<p<5, \\ (1-\beta) \frac{p-1}{3+p} & \text { for } 5 \leq p .\end{cases}
$$

Then, there is $C=C(t)$ bounded on finite time intervals such that $C(0)=C_{0}$ depends only on $\Omega$ and for all $q^{*} \in W_{p}^{2,1}\left(Q_{t}\right)$

$$
\left.\left\|q^{*}\right\|_{C^{\beta, \frac{\beta}{2}}\left(Q_{t}\right)} \leq\left\|q^{*}(0)\right\|_{C^{\beta}(\Omega)}+C(t) t^{\gamma}\left[\left\|q^{*}\right\|_{W_{p}^{2,1}\left(Q_{t}\right)}+\left\|q^{*}\right\|_{C\left([0, t] ; W_{p}^{2-\frac{2}{p}}(\Omega)\right.}\right)\right] .
$$

Finally we have a perturbation Lemma for elliptic problems. This property ought to be well known, and we only mention details for more convenience on reading.

Lemma 28. Let $a \in C^{\beta}(\bar{\Omega})(\beta>0)$ satisfy $0<a_{0} \leq a(x) \leq a_{1}<+\infty$ for all $x \in \Omega$. Suppose that $F \in L^{p}(\Omega)$ with $p>3$. Then, there is a unique $u \in W^{1, p}(\Omega)$ satisfying $\int_{\Omega}(a(x) \nabla u-F(x)) \cdot \nabla \phi d x=0$ for all $\phi \in C^{1}(\bar{\Omega})$ and $\int_{\Omega} u d x=0$. Moreover, there is $c=c\left(\Omega, p, a_{0}, a_{1}\right)$ such that

$$
\|\nabla u\|_{L^{p}(\Omega)} \leq c\left(1+[a]_{C^{\beta}}\right)^{\frac{1}{\beta}}\|F\|_{L^{p}(\Omega)} .
$$


Proof. Existence of a unique weak solution is well-known. In order to prove the estimate, we start recalling a few standard inequalities. First, the bound $\sqrt{a_{0}}\|\nabla u\|_{L^{2}} \leq$ $\|F\|_{L^{2}}$ is valid. Since we choose the mean-value of $u$ to be zero, then also $\|u\|_{L^{2}} \leq$ $c_{\Omega} a_{0}^{-\frac{1}{2}}\|F\|_{L^{2}}$. Moreover, for $s>3$ arbitrary, we find that $\|u\|_{L^{\infty}(\Omega)} \leq c(\Omega, s)\left(a_{0}^{-1}\right.$ $\left.\|F\|_{L^{s}(\Omega)}+\frac{a_{1}}{a_{0}}\|u\|_{L^{s / 2}(\Omega)}\right)$. Thus, choosing $s \leq \min \{p, 4\}$ and employing the Hoelder inequality, we easily show that $\|u\|_{L^{\infty}(\Omega)} \leq \tilde{c}\left(\Omega, a_{0}, a_{1}\right)\|F\|_{L^{p}(\Omega)}$.

We now come to the main argument. We consider $x^{0}$ in $\Omega$ and $r>0$. We choose a nonnegative cut-off function $\eta \in C_{c}^{1}\left(B_{r}\left(x^{0}\right)\right)$ satisfying $|\nabla \eta| \leq c_{0} r^{-1}$. Choosing in the weak formulation a testfunction of the form $\phi \eta$, we obtain, after some obvious shifting, for $w:=u \eta$ the identity

$$
\begin{aligned}
& a\left(x^{0}\right) \int_{\Omega} \nabla w \cdot \nabla \phi d x=\int_{\Omega}\left(a\left(x^{0}\right)-a\right) \nabla w \cdot \nabla \phi d x \\
& \quad+\int_{\Omega}(F \eta+a u \nabla \eta) \cdot \nabla \phi d x+\int_{\Omega}(F \cdot \nabla \eta+a \nabla \eta \cdot \nabla u) \phi d x .
\end{aligned}
$$

This is a weak Neumann problem for the Laplacian of $w$. By standard results, we obtain an estimate

$$
\begin{aligned}
& \|\nabla w\|_{L^{p}} \leq c(\Omega, p)\left(\left\|\left(1-\frac{a}{a\left(x^{0}\right)}\right) \nabla w\right\|_{L^{p}}\right. \\
& \left.\quad+\frac{1}{a\left(x^{0}\right)}\left(\|F \eta+a u \nabla \eta\|_{L^{p}}+\left\|F \cdot \nabla \eta+a \nabla \eta \cdot \nabla u+a\left(x^{0}\right) w\right\|_{L^{\frac{p^{*}}{p^{*}}(\Omega)}}\right)\right) .
\end{aligned}
$$

Here $p^{*}$ is the Sobolev embedding exponent of $W^{1, p}(\Omega)$. For $p>3$, we have $p^{*}=$ $+\infty$ and $p^{*} /\left(p^{*}-1\right)=1$. Next, since $w$ is supported in $B_{r}\left(x^{0}\right)$, and since $a$ is Hoelderian, it follows that

$$
\left\|\left(1-\frac{a}{a\left(x^{0}\right)}\right) \nabla w\right\|_{L^{p}} \leq \frac{[a]_{C^{\beta}}}{a\left(x^{0}\right)} r^{\beta}\|\nabla w\|_{L^{p}} .
$$

Thus, fixing $r^{\beta}:=\frac{a_{0}}{2 c(\Omega, p)[a]_{C} \beta}$, we obtain that

$\|\nabla w\|_{L^{p}} \leq \frac{2 c(\Omega, p)}{a\left(x^{0}\right)}\left(\|F \eta+a u \nabla \eta\|_{L^{p}}+\left\|F \cdot \nabla \eta+a \nabla \eta \cdot \nabla u+a\left(x^{0}\right) w\right\|_{L^{1}(\Omega)}\right)$.

With the notation $\Omega_{r}\left(x^{0}\right)=B_{r}\left(x^{0}\right) \cap \Omega$, we notice that

$$
\begin{aligned}
\|\nabla w\|_{L^{p}} & \geq\|\nabla u \eta\|_{L^{p}}-\frac{c_{0}}{r}\|u\|_{L^{p}\left(\Omega_{r}\left(x^{0}\right)\right)}, \\
\|F \eta+a u \nabla \eta\|_{L^{p}} & \leq\|F \eta\|_{L^{p}}+\frac{a_{1} c_{0}}{r}\|u\|_{L^{p}\left(\Omega_{r}\left(x^{0}\right)\right)}, \\
\left\|F \cdot \nabla \eta+a \nabla \eta \cdot \nabla u+a\left(x^{0}\right) w\right\|_{L^{1}} & \leq \frac{c_{0}}{r}\left(\|F\|_{L^{1}\left(\Omega_{r}\left(x^{0}\right)\right)}+a_{1}\|\nabla u\|_{L^{1}\left(\Omega_{r}\left(x^{0}\right)\right)}\right) .
\end{aligned}
$$

Thus, we have shown that

$$
\begin{aligned}
\|\nabla u \eta\|_{L^{p}} \leq & \frac{2 c(\Omega, p)}{a_{0}}\left(\|F \eta\|_{L^{p}}+\frac{c_{0}}{r}\left(\|F\|_{L^{1}\left(\Omega_{r}\left(x^{0}\right)\right)}+a_{1}\|\nabla u\|_{L^{1}\left(\Omega_{r}\left(x^{0}\right)\right)}\right)\right) \\
& +\frac{c_{0}}{r}\left(1+2 c(\Omega, p) \frac{a_{1}}{a_{0}}\right)\|u\|_{L^{p}\left(\Omega_{r}\left(x^{0}\right)\right)} .
\end{aligned}
$$


By appropriate covering of $\Omega$ with partition of unity, we obtain the inequality

$$
\begin{aligned}
\|\nabla u\|_{L^{p}} \leq & \frac{2 m_{0} c(\Omega, p)}{a_{0}}\|F\|_{L^{p}}+\frac{2 c_{0} m_{0} c(\Omega, p)}{a_{0} r}\|F\|_{L^{1}} \\
& +\frac{a_{1} c_{0}}{a_{0} r} m_{0}\left(2 c(\Omega, p)\|\nabla u\|_{L^{1}}+(1+2 c(\Omega, p))\|u\|_{L^{p}}\right) .
\end{aligned}
$$

Here $m_{0}$ is some geometric constant associated with the covering of $\Omega$. It remains to estimate

$$
\begin{gathered}
\|\nabla u\|_{L^{1}} \leq|\Omega|^{\frac{1}{2}}\|\nabla u\|_{L^{2}} \leq|\Omega|^{\frac{1}{2}} a_{0}^{-\frac{1}{2}}\|F\|_{L^{2}} \\
\|u\|_{L^{p}} \leq|\Omega|^{\frac{1}{p}}\|u\|_{L^{\infty}(\Omega)} \leq c(\Omega, p)\|F\|_{L^{p}},
\end{gathered}
$$

where we employ the preliminary consideration at the beginning of this proof to show that $\|u\|_{L^{\infty}} \leq c\|F\|_{L^{p}}$. Recalling the choice of $r$, we are done.

\section{REFERENCES}

[1] D. Bothe. On the Maxwell-Stefan approach to multicomponent diffusion. In Progress in Nonlinear differential equations and their Applications 80, pages 81-93. Springer, 2011.

[2] D. Bothe and W. Dreyer. Continuum thermodynamics of chemically reacting fluid mixtures. Acta Mech., 226:1757-1805, 2015.

[3] D. Bothe, W. Dreyer, and P.-E. Druet. Multicomponent incompressible fluids - An asymptotic study. Preprint, 2021. Available at http://www.wias-berlin.de/preprint/2825/wias_preprints_2825. pdf, and at arXiv:2104.08628 [math-ph].

[4] D. Bothe and P.-E. Druet. On the structure of continuum thermodynamical diffusion fluxes: a novel closure scheme and its relation to the Maxwell-Stefan and the Fick-Onsager approach. Preprint, 2020. Available at: http://www.wias-berlin.de/preprint/2749/wias_preprints_2749.pdf and at arXiv:2008.05327 [math-ph].

[5] D. Bothe and P.-E. Druet. Mass transport in multicomponent compressible fluids: local and global well-posedness in classes of strong solutions for general class-one models. Nonlinear Analysis, 210:112389, 2021. https://doi.org/10.1016/j.na.2021.112389.

[6] D. Bothe and J. Prüss. Modeling and analysis of reactive multi-component two-phase flows with mass transfer and phase transition - the isothermal incompressible case. Discrete Contin. Dyn. Syst. Ser. S, 10:673-696, 2017.

[7] D. Bothe and K. Soga. Thermodynamically consistent modeling for dissolution/growth of bubbles in an incompressible solvent. In Amann H., Giga Y., Kozono H., Okamoto H., Yamazaki M. (eds) Recent Developments of Mathematical Fluid Mechanics, Advances in Mathematical Fluid Mechanics. Birkhäuser, Basel, 2016.

[8] X. Chen and A. Jüngel. Analysis of an incompressible Navier-Stokes-Maxwell-Stefan system. Commun. Math. Phys., 340:471-497, 2015.

[9] S. R. deGroot and P. Mazur. Non-Equilibrium Thermodynamics. North Holland, Amsterdam, 1963.

[10] A. Donev, A. Nonaka, A.K. Bhattacharjee, A.L. Garcia, and J.B. Bell. Low Mach number fluctuating hydrodynamics of multispecies liquid mixtures. Physics of Fluids, 27:97-112, 2015. https://doi.org/ 10.1063/1.4913571.

[11] W. Dreyer, P.-E. Druet, P. Gajewski, and C. Guhlke. Existence of weak solutions for improved Nernst-Planck-Poisson models of compressible reacting electrolytes. Z. Angew. Math. Phys., 71(119), 2020. Open access. https://doi.org/10.1007/s00033-020-01341-5.

[12] W. Dreyer, C. Guhlke, and R. Müller. Overcoming the shortcomings of the Nernst-Planck model. Phys. Chem. Chem. Phys., 15:7075-7086, 2013.

[13] W. Dreyer, C. Guhlke, and R. Müller. Bulk-surface electro-thermodynamics and applications to electrochemistry. Entropy, 20:939/1-939/44, 2018. DOI https://doi.org/10.3390/e20120939. 
[14] P.-E. Druet. Global-in-time existence for liquid mixtures subject to a generalised incompressibility constraint. J. Math. Analysis and Appl., 499, 2021. doi:https://doi.org/10.1016/j.jmaa.2021.125059.

[15] E. Feireisl, Y. Lu, and J. Málek. On PDE analysis of flows of quasi-incompressible fluids. Z. Angew. Math. Mech., 96:491-508, 2016.

[16] V. Giovangigli. Multicomponent Flow Modeling. Birkhäuser, Boston, 1999.

[17] H. Gouin, A. Muracchini, and T. Ruggeri. On the müller paradox for thermal-incompressible media. Continuum Mech. Thermodyn., 24:505-513, 2012.

[18] M. Herberg, M. Meyries, J. Prüss, and M. Wilke. Reaction-diffusion systems of Maxwell-Stefan type with reversible mass-action kinetics. Nonlinear Analysis: Theory, Methods \& Applications, 159:264-284, 2017.

[19] D.D. Joseph, A. Huang, and H. Hu. Non-solenoidal velocity effects and Korteweg stresses in simple mixtures of incompressible liquids. Physica D, 97:104-125, 1996.

[20] A. Jüngel and I. Stelzer. Existence analysis of Maxwell-Stefan systems for multicomponent mixtures. SIAM J. Math. Anal., 45:2421-2440, 2013.

[21] Ladyzenskaja, Solonnikov, and Ural'ceva. Linear and Quasilinear Equations of Parabolic Type, volume 23 of Translations of mathematical monographs. AMS, 1968.

[22] M. Marion and R. Temam. Global existence for fully nonlinear reaction-diffusion systems describing multicomponent reactive flows. J. Math. Pures Appl., 104:102-138, 2015.

[23] J. Meixner and H. G. Reik. Thermodynamik der irreversiblen Prozesse, volume 3, pages 413-523. Springer, Berlin, 1959. German.

[24] N. Mills. Incompressible mixtures of Newtonian fluids. Int. J. Engng Sci., 4:97-112, 1966.

[25] I. Müller. Thermodynamics. Pitman, London, 1985.

[26] L. Nirenberg. An extended interpolation inequality. Annali della Scuola Normale Superiore di Pisa (3), 20:733-737, 1966.

[27] M. Pekař and I. Samohýl. The Thermodynamics of Linear Fluids and Fluid Mixtures. Springer International Publishing Switzerland, 2014.

[28] R. T. Rockafellar. Convex Analysis. Princeton University Press, Princeton, New Jersey, 1970.

[29] V.A. Solonnikov. Solvability of the initial-boundary-value problem for the equations of motion of a viscous compressible fluid. J. Math. Sci., 14:1120-1133, 1980. https://doi.org/10.1007/ BF01562053.

\author{
Dieter Bothe \\ Mathematische Modellierung und Analysis \\ Technische Universität Darmstadt \\ Alarich-Weiss-Str. 10 \\ 64287 Darmstadt \\ Germany \\ E-mail:bothe@mma.tu-darmstadt.de \\ Pierre-Etienne Druet \\ Weierstrass Institute \\ Mohrenstr. 39 \\ 10117 Berlin \\ Germany \\ E-mail: pierre-etienne.druet@wias-berlin.de
}

